\title{
A Diagnostic Technique for Particle Characterization using Laser Light
}

\section{Extinction}

Kris Barboza

Thesis submitted to the faculty of the Virginia Polytechnic Institute and State University in partial fulfillment of the requirements for the degree of

Master of Science

In

Mechanical Engineering

Srinath V. Ekkad Chair

Wing F. Ng Co-Chair

K. Todd Lowe

Walter F. O’Brien

April 17, 2015

Blacksburg, VA

Keywords: Optical Diagnostics, On-board Sensor, Light Extinction, Particle Characterization, Mie Extinction 


\title{
A Diagnostic Technique for Particle Characterization using Laser Light
}

\section{Extinction}

Kris Barboza

\begin{abstract}
Increased operations of aircraft, both commercial and military, in hostile desert environments have increased risks of micro-sized particle ingestion into engines. The probability of increased sand and dust ingestion results in increased life cycle costs, in addition to increased potential for performance loss. Thus, abilities to accurately characterize inlet sand would be useful for engine diagnostics and prognostic evaluation. Previous characterization studies were based on particle measurements performed a posteriori. Thus, there exists a need for in situ quantification of ingested particles.

The work presented in this thesis describes initial developments of a line-of-sight optical technique to characterize ingested particles at concentrations similar to those experienced by aircraft in brownout conditions using light extinction with the end goal of producing an onboard aircraft diagnostic sensor. By measuring the extinct light intensity in presence of particles over range of concentrations, a relationship between diameters, concentration and light extinction was used for characterization. The particle size distribution was assumed log-normal and size range of interest $1-10 \mu \mathrm{m}$.

To validate the technique, particle characterization in both static and flow based tests were performed on polystyrene latex spheres of sizes $1.32 \mu \mathrm{m}, 3.9 \mu \mathrm{m}, 5.1 \mu \mathrm{m}$, and $7 \mu \mathrm{m}$ in monodisperse and poly-disperse mixtures. Results from the static experiments were obtained with a maximum relative error of $11 \%$. Concentrations from the static experiments were obtained with a maximum relative error of 18\%. Mono-dispersed and poly-dispersed particle samples were
\end{abstract}


sized in a flow setup, with a maximum relative error of $12 \%$ and $10 \%$ respectively across all diameter samples tested. Uncertainty in measurements were quantified, with results indicating a maximum error of $17 \%$ in diameters due to sources of variability and showed that shorter wavelength lasers provide lower errors in concentration measurements, compared to longer wavelengths.

For real time, on-board measurements, where path lengths traveled by light are much larger than distances traveled in initial proof of concept experimental setups, requirements would be to install sensitive detectors and powerful lasers to prevent operation near noise floors of detectors. Vibration effects from the engine can be mitigated by using larger area collection optics to ensure that the transmitted light falls on active detector areas.

Results shown in this thesis point towards validity of the light extinction technique to provide real time characterization of ingested particles, and will serve as an impetus to carry out further research using this technique to characterize particles entering aircraft engine inlets. 
Dedicated to Nicole whose love, encouragement, support and honesty has made this possible for me. 


\section{Acknowledgments}

I would like to take this opportunity to thank all the following individuals without whom this work could not have been conceived. I would like to express my gratitude to Dr. Srinath Ekkad and Dr. Wing Ng who have been instrumental in my professional development. Your constant guidance and support has greatly enriched my research experience. Thank you for your confidence in me.

I would also like to thank Dr. Todd Lowe, Dr. Lin Ma and Dr. Walter O'Brien who have greatly helped me throughout the course of this work with their extremely valuable insights. A special thanks is due to David Gomez, Dr. Ryan Blanchard and Dr. A.J Wickersham for sharing their invaluable experiences in designing and installing optical experiments. This work would not have been possible without the support and inputs from Rolls-Royce North America.

I am indebted to the entire HEFT lab team, Sridharan Ramesh, Dr. Jaideep Pandit, Kartikeya, Prashant, Sandip, Hardik, Bharath, Sammrudhi, Siddharth, Prtihvi whose expertise and constant discussion provided a fresh perspective on my research. Special thanks to the RollsRoyce student team, Raul, Chu and Suhyeon for constantly motivating me to work harder. I would also like to thank Diana Israel and Mandy Collins for the administrative support throughout the course of this project.

Finally and most importantly, I would like to acknowledge my family, Mai, Pappa, Mom, Dad, Kevin and Sheeja, for all their support and guidance. 


\section{Table of Contents}

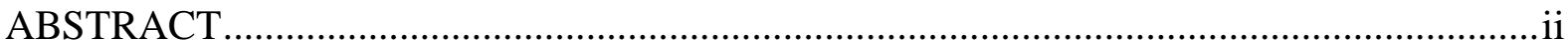

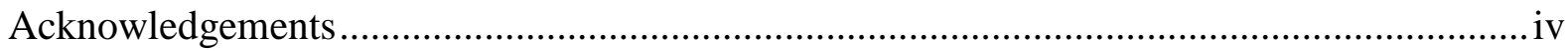

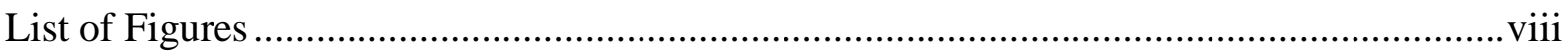

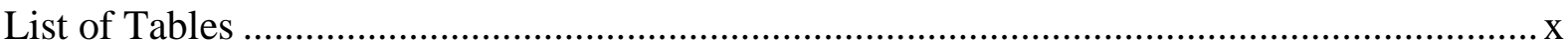

Preface

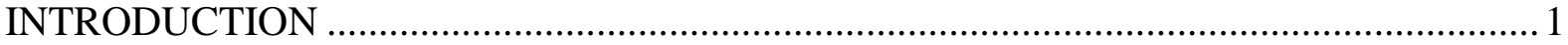

Motivation for Inlet Particle Measurements ..................................................................... 1

Optical Techniques for Aerosol Characterization............................................................... 4

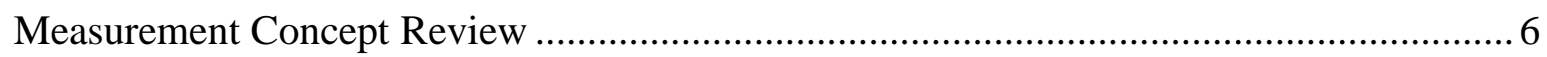

PROOF OF CONCEPT EXPERIMENT 1: STATIC SETUP ….......................................... 14

Brownout Landings and Sand Particles Entrained........................................................... 14

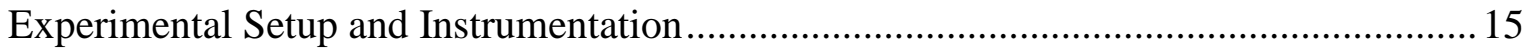

Results from the Static Setup (Mono-dispersed Samples)................................................. 19

Measurements of Diameters and Concentrations in a Poly-dispersed Sample................... 26

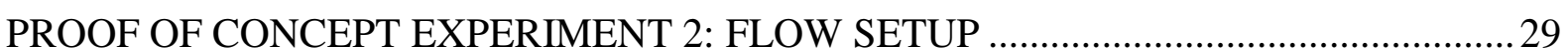

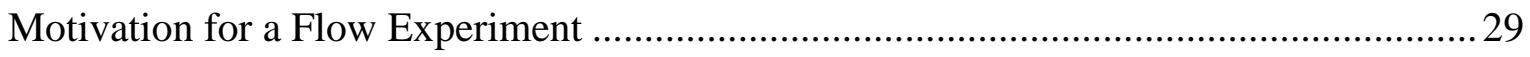

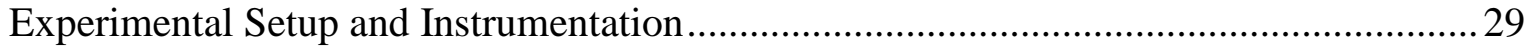

Results from the Flow Setup (Mono-dispersed Samples)................................................ 33

Measurements of Diameters in a Poly-dispersed Sample ................................................. 36

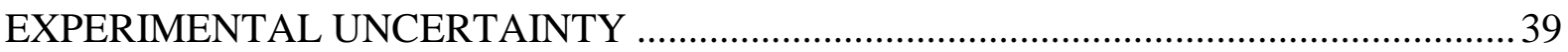

Dependence of Diameters and Concentrations on Measured Parameters .......................... 39

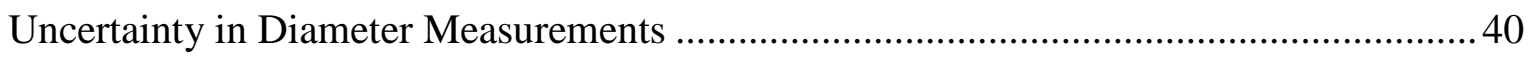

Uncertainty in Concentration Measurements .............................................................. 43

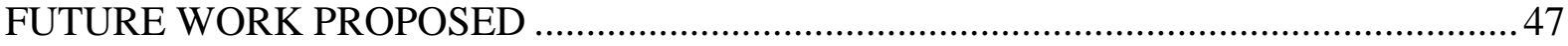

Simultaneous Particle Diameter and Concentration Measurements in Flows .................... 47

Extension of Technique to Water Aerosols and Silica Particles......................................... 49

Possible Sources of Error in Future On-Board Measurements ..........................................52

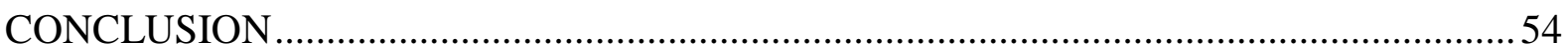

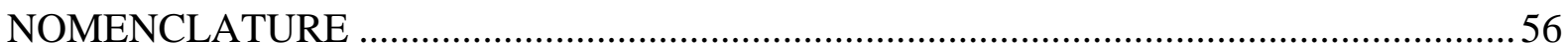

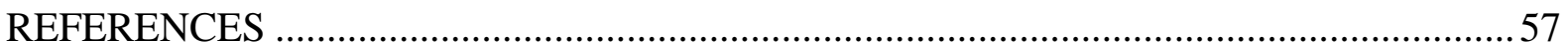


APPENDICES

60

Appendix A: Disambiguation of Diameters Obtained ........................................................ 60

Appendix B: Codes for Mie Extinction Applied to Particle Size Distributions 62 


\section{List of Figures}

Figure 1: Effect of particulate matter ingestion on gas turbine components

Figure 2: Particle deposition of bituminous fly ash shown on TBC coupons following immediate shutdown (top) and cool down to room temperature (bottom)

Figure 3: Interaction of light with a small particle describing the phenomenon of scattering and extinction.

Figure 4: $\overline{\mathrm{Q}}$ at two distinct wavelengths vs diameter for increasing distribution widths

Figure 5: Ratio of $\bar{Q}$ using $450 \mathrm{~nm}$ and $7000 \mathrm{~nm}$ lasers at 5 distribution widths (standard deviations) using polystyrene particles

Figure 6: Variation of $\mathrm{R}$ versus distribution width for a diameter of $8 \mu \mathrm{m}$ using combination of $450 \mathrm{~nm}$ and $7000 \mathrm{~nm}$ lasers resulting in a distribution width prediction of 1.2

Figure 7: Schematic representation of static experimental setup.

Figure 8: Flow chart illustrating the measurement technique utilized

Figure 9: Diameter vs concentration measured compared with actual values for sampled polystyrene particles.

Figure 10: Concentration measured using $450 \mathrm{~nm}$ light for $1.32 \mu \mathrm{m}$ particles .22

Figure 11: Concentration measured using $635 \mathrm{~nm}$ light for $3.9 \mu \mathrm{m}$ particles 22

Figure 12: Concentration measured using $450 \mathrm{~nm}$ light for $5.1 \mu \mathrm{m}$ particles 23

Figure 13: Standard deviation vs ratio of transmissivities for $5 \mu \mathrm{m}$ particles.

Figure 14: Particle size distribution for $1.32 \mu \mathrm{m}$ polystyrene latex 24

Figure 15: Particle size distribution for $3.9 \mu \mathrm{m}$ polystyrene latex 25

Figure 16: Particle size distribution for $5.1 \mu \mathrm{m}$ polystyrene latex 25

Figure 17: Particle diameter measured for mixed samples .27

Figure 18: Particle concentration measured for mixed sample of mean diameter $1.89 \mu \mathrm{m} \ldots 27$

Figure 19: Particle concentration measured for mixed sample of mean diameter $4.3 \mu \mathrm{m} \ldots . .28$

Figure 20: Test schematic of the second bench-top experiment to size particles in flows ..... 30

Figure 21: Actual picture of second bench-top experiment used to size particles in flows ... 30

Figure 22: Optical setup used in the second experiment used to size particles in flows ........ 32 
Figure 23: Histogram of $3.9 \mu \mathrm{m}$ particles measured at velocities under $5 \mathrm{~m} / \mathrm{s}$

Figure 24: Histogram of $5.1 \mu \mathrm{m}$ particles measured at velocities under $5 \mathrm{~m} / \mathrm{s}$ 34

Figure 25: Histogram of $7 \mu \mathrm{m}$ particles measured at velocities under $5 \mathrm{~m} / \mathrm{s}$ .35

Figure 26: Histogram of poly disperse particle samples with a mean diameter of $4.41 \mu \mathrm{m} . .37$

Figure 27: Histogram of poly disperse particle samples with a mean diameter of $5 \mu \mathrm{m}$ .37

Figure 28: Histogram of poly disperse particle samples with a mean diameter of $6.01 \mu \mathrm{m} . .38$

Figure 29: Error in diameter measurement due to sources of uncertainty using the current setup of $447 \mathrm{~nm}$ and $635 \mathrm{~nm}$ lasers

Figure 30: Error in diameter measurement due to sources of uncertainty using $447 \mathrm{~nm}$ and $7000 \mathrm{~nm}$ lasers to be used in the future.

Figure 31: Plot showing the ratio of transmissivity (R) vs. diameters demonstrating the effect of refractive index variation on diameter measurements for $447 \mathrm{~nm}$ and $7000 \mathrm{~nm}$ lasers ...... 43

Figure 32: Percentage errors in concentration measurements due to various sources of uncertainty using $447 \mathrm{~nm}, 635 \mathrm{~nm}$ and $7000 \mathrm{~nm}$ lasers.

Figure 33: Schematic of the flow setup modified to obtain concentration validation measurements using a camera for image analysis 48

Figure 34: Actual image of the flow setup modified to obtain concentration validation measurements using a camera for image analysis

Figure 35: Ratio of extinction efficiencies for combinations of $635 \mathrm{~nm}, 450 \mathrm{~nm}$ and $7000 \mathrm{~nm}$ lasers using water aerosols.

Figure 36: Ratio of extinction efficiencies for combinations of 450nm and 7000nm lasers using silica particles

Figure A1: Ratio of transmissivity R versus diameter using $635 \mathrm{~nm}$ and $450 \mathrm{~nm}$ laser for a standard deviation of 1.033

Figure A2: Ratio of transmissivity $\mathrm{R}$ vs diameter using $450 \mathrm{~nm}$ and $10000 \mathrm{~nm}$ laser for a standard deviation of 1.033 . 


\section{List of Tables}

Table 1: Particle characterization of a brownout cloud for different airframes. Source, Defense Advanced Research Projects Agency (DOD) Strategic Technology Office. Issued by U.S. Army Aviation and Missile Command.....

Table 2: Maximum and minimum concentration of particles sampled in the static setup ..... 13 


\section{Preface}

The work in this thesis describes the initial development of a sensor to determine the diameters and concentration of particulate matter entering an aircraft engine inlet. The author of the thesis was responsible for designing and obtaining data from proof of concept experiments that were set up to gauge the performance of the laser light extinction technique in characterizing particulate matter which would eventually find its application as a prognostic evaluation tool in aircraft engine inlets.

This thesis begins with a motivation for the measurement along with an introduction to the laser extinction technique along with the measurement concept involved. This is followed by a description of the first bench top experiment performed along with a discussion of results from the same. A second proof of concept bench top experiment is then described along with results from measurements. Uncertainties arising from this technique are quantified. A short discussion of extending the technique to realistic conditions along with sources that may result in an erroneous signal are discussed. This is finally followed by a conclusion highlighting the key successes from the proof of concept experiments. 


\section{INTRODUCTION}

\section{Motivation for Inlet Particle Measurements}

Increased operations of aircraft in hostile desert environments poses a serious threat to gas turbine engines. The increased number of hubs near desert environments may result in ingestion of large quantities of particulate matter in the engine during sandstorms. A serious degraded visual environment (DVE) called brownout is caused due to the downwash created by Turboshaft engine rotors while they are in operation. This results in ingestion of huge quantities of sand particulate matter into the engine. Quantities of up to $4.86 \times 10^{11}$ particles per unit volume have been measured in experiments.

This particulate matter is ingested in short bursts with large concentrations and causes severe damage to engine turbomachinery. During take-off, engines are operated at elevated temperatures and mass flow rates. The ingested particles enter the engine through the compressor and deposit themselves on the compressor causing a change in blade profile and subsequent erosion. As the remaining particulate-air mixture travels through the compressor, the air density increases but the sand density does not increase. This results in an increased sand to air volume ratio.

In many locations, air is bled through the combustor bypass to provide cooling for turbine components. This air is used for convective cooling in internal passages and is directed through holes for film cooling. As the sand has a lower velocity than the air, slip velocity between sand and air is small and the particulate temperature increases rapidly through radiation thereby increasing the probability of deposition on coolant passages. In gas turbines, it is critical that the coolant flow rate be maintained at a specified amount. Any blockage in the passages results in a reduced flow rate and the component cannot be cooled adequately resulting in localized hot spots and consequently increased thermal stresses. 
In addition to cooling deterioration, the ingested sand causes fouling of combustors, and deposition on internal shafts, resulting in an imbalance of the rotating components causing unwanted and unnecessary vibrations. The ingested sand results in reduced thermal and aerodynamic performance, increased engine down time, increased life cycle costs and in most severe cases, complete failure of engine components while in operation resulting in loss of personnel. Scheduled engine overhaul costs range from $\$ 160,000$ to $\$ 350,000$ per engine while unscheduled engine repair costs $\$ 70,000$. These elevated unscheduled costs can be reduced by curtailing operations when the ingested sand particulate matter is high. Thus there exists a need for a prognostic engine health evaluation tool that would provide ingested particulate matter characteristics to reduce engine damage.

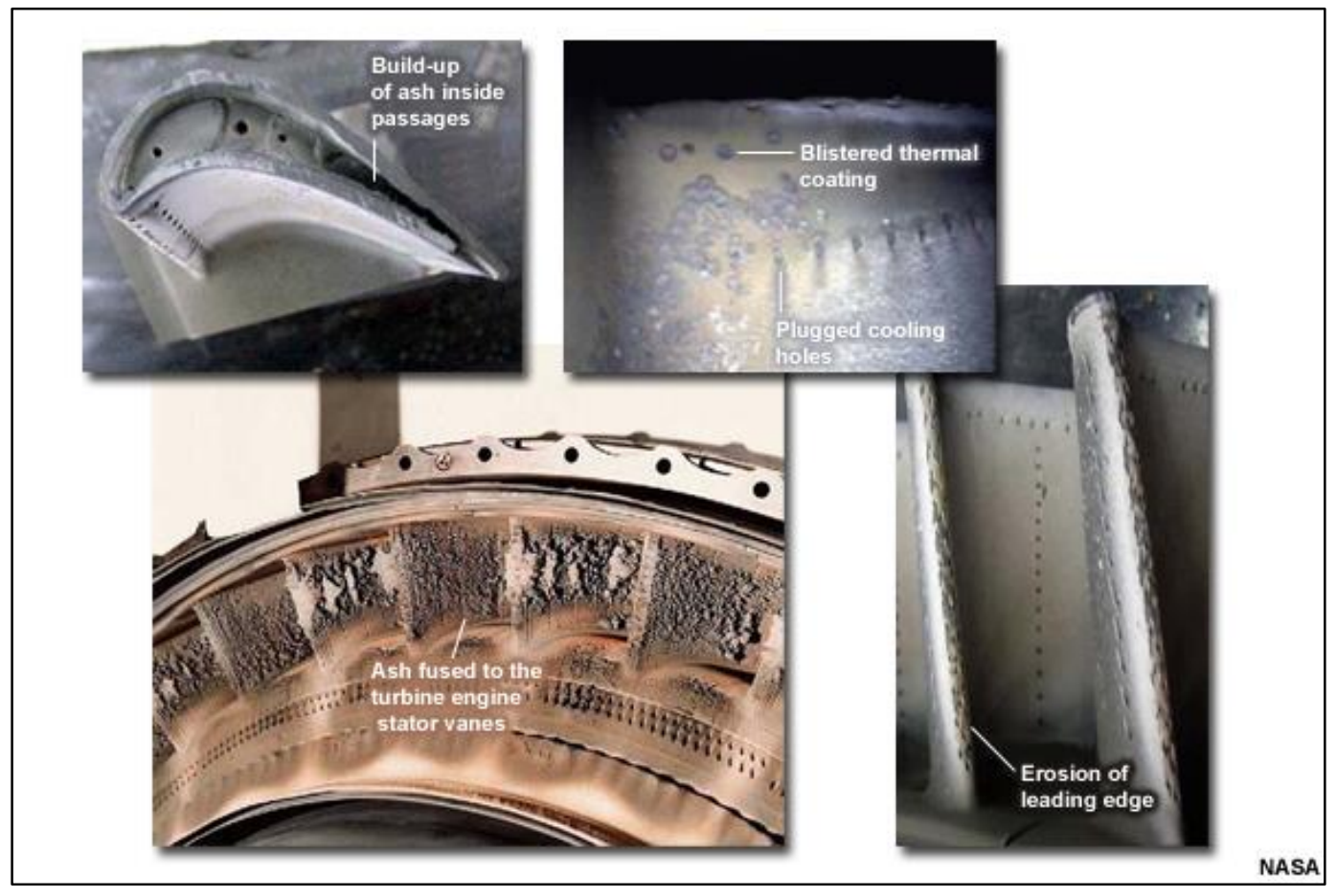

Figure 1: Effect of particulate matter ingestion on gas turbine components Source: http://www.goes-r.gov/users/comet/volcanic_ash/impacts/print.htm 

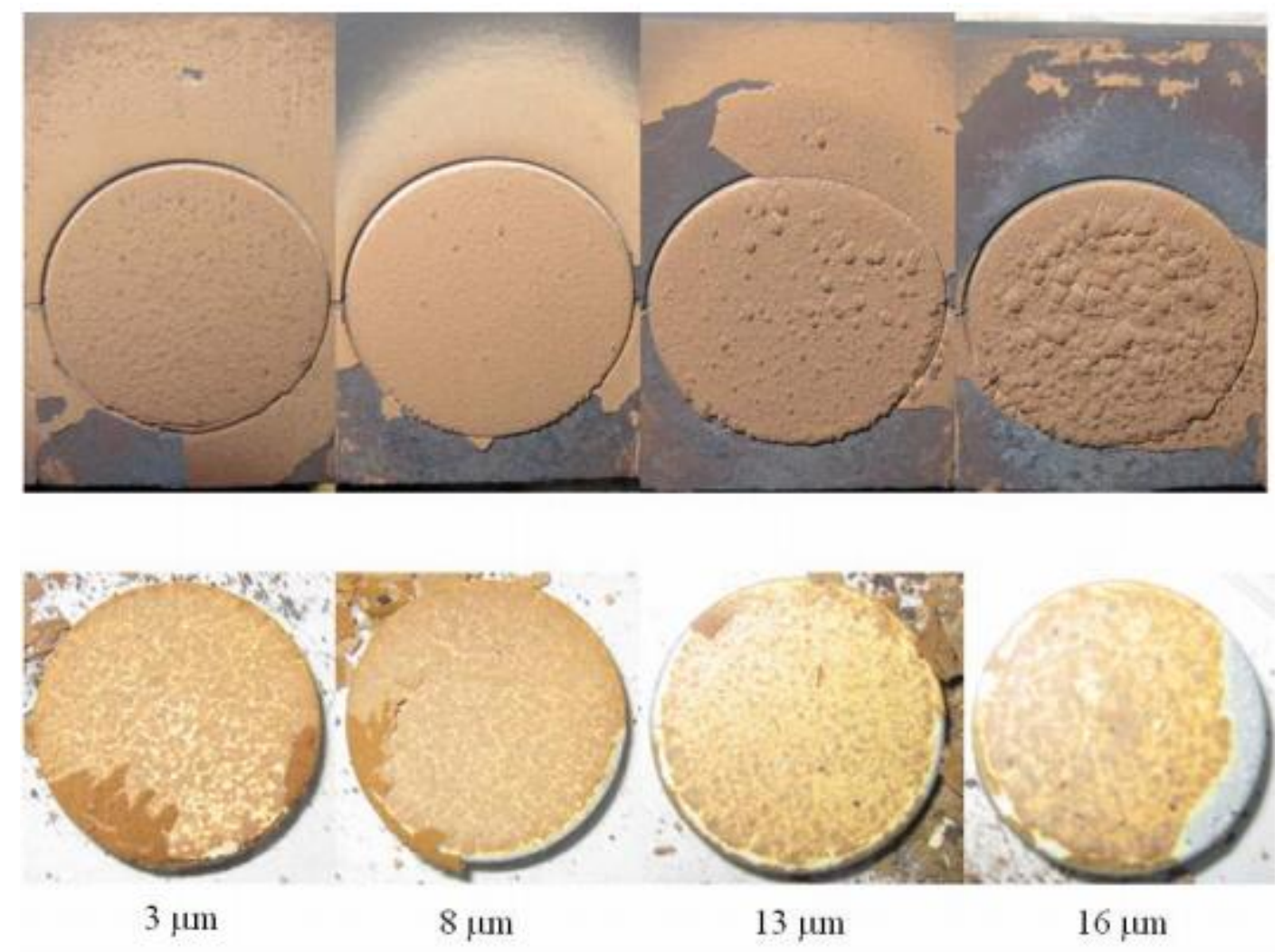

Figure 2: Particle deposition of bituminous fly ash shown on TBC coupons following immediate shutdown (top) and cool down to room temperature (bottom) NOTE: Airflow is into the page and temperature was $1183^{\circ} \mathrm{C}$

Source: http://www.et.byu.edu/ tom/Papers/Crosby_Thesis.pdf

The prognostic evaluation tool should have the following qualities:

1. Non - intrusive measurement

2. Excellent temporal and spatial resolution

3. Real time data capability

4. Low instrumentation weight

5. Low resource utilization

6. Ease of installation 


\section{Optical Techniques for Aerosol Characterization}

Optical techniques permit rapid and non-invasive abilities to measure properties of aerosols [1]. The main advantage of optical techniques is the possibility of performing measurements of optical properties in situ and in real time. The measured optical properties are influenced by physical properties of aerosols and hence information such as diameters of particles can be extracted from these measurements [2].

Many optical techniques are available for particle sizing. Some of these techniques include Phase Doppler Systems, Interferometric Particle Sizing, Time-shift Backscatter, Rainbow Refractometry, Laser Diffraction, Multi-angle Scattering and Mie Extinction. Characterization of small particles by light scattering techniques usually require intensity measurements at multiple angles [3-6]. However these techniques involve extensive utilization of resources, are complex by nature and may not be suitable for high concentrations of particles in the sampling volume[7-8]. Phase Doppler systems have complicated optics to collect the scattered light and are usually performed on single particles in a limited sampling volume which may not be possible in flows with high particle concentrations. Rainbow refractometry requires precision optical positioning to obtain the rainbow ray and positioning these optics may be cumbersome especially in aircraft engine inlets. Among all optical methods for measurements of size distribution, techniques based on the line of sight Mie extinction are attractive due to their simplicity in implementation, capability to provide continuous measurement with high temporal resolution and very limited requirement of optical access [9], all of which are requirements of an onboard aircraft diagnostic sensor.

Experiments incorporating the principle of Mie extinction have been performed in the past by R.Ltichford et al to measure soot particulate loading in the exhaust stream of gas turbine engines [10]. 17 wavelengths with a uniform spacing of $25 \mathrm{~nm}$ were chosen and the particle size range of interest in these measurements was 0.04-0.06 $\mu \mathrm{m}$ [10]. Smolders, Dongen, Barun 
and Snoeijs studied time dependent droplet sizing based on spectral extinction by measuring the extinction coefficients at three different wavelengths using a spectrometer and a CCD array and applied an inversion technique based on trial size distributions [11]. Ramachandran and Leith developed an algorithm to obtain particle size and concentration measurements of particles between $0.3 \mu \mathrm{m}$ and $2.5 \mu \mathrm{m}$. An information content parameter was used to measure how well each size distribution could be reconstructed from the corresponding measurements [12].

Eberle, Schatz, Grubel et al studied the size and number of droplets of steam in low pressure turbines by obtaining extinction measurements at multiple wavelengths and replacing the extinction equations with a quadrature scheme yielding a linear equation that could be written in a matrix form [13]. However the linear equation obtained is an ill conditioned equation requiring extensive mathematical rigor to obtain meaningful measurement data. Cai and Wang [14] performed extinction measurements to determine the size distribution of particles and used 8 light wavelengths from 0.4 to $0.85 \mu \mathrm{m}$ to obtain measurements. The partial Lagrange multipliers non-negative least squares algorithm (PLMNNLS) was used to solve the ill conditioned equation arising from the extinction measurements at the 8 wavelengths of light used.

Measurement performed in the past as described above to determine size distributions by light extinction relied on light sources from a relatively narrow wavelength range ranging from the near UV to the near IR [8-12] which poses two problems. First, the amount of information that can be inferred from extinction measurements is very limited if the spectral range of light wavelengths used is narrow (less than $50 \mathrm{~nm}$ ). Second, extinction measurements in a narrow spectral range limit the sensitivity of size distribution measurements and hence the applicable range of the method [2]. This is due to the fact that measurements made using the smaller 
wavelength spectral range are more susceptible to experimental errors as the orthogonal functions for the shorter spectral gap have fine structures and more oscillations [12].

The rapid development of laser technologies facilitates the possibility of utilizing light sources in a wider spectral range for the extinction method. The availability of detectors with excellent signal to noise ratios over a large spectral range coupled with rise times of nanoseconds allow measurements of aerosol particle properties in flow streams. Combining advancements in these technologies permits particle size distribution measurements in aircraft engine inlets. The results and discussions presented in this thesis will serve as a fundamental step in validating the usage of light extinction techniques for real time measurements of particle size distributions. As an initial proof of concept to validate this method and to keep the cost of experimentation low, two bench top experiments (static and flow) to measure particle sizes were performed using polystyrene particles of a size range 1-10 $\mu \mathrm{m}$ dispersed in distilled water. Two light sources, 180nm spectrally apart were used to obtain information on diameters and consequently number densities. On completion of sizing, the discussion is extended to the possibility of real time measurement of water aerosols and silica particles in aircraft engine inlets.

\section{Measurement Concept Review}

When a beam of light strikes a particle, it may be scattered and absorbed depending on the particle's physical composition and size. The combined effect of scattering and absorption along the beam propagation direction results in an intensity loss in the light beam. This effect is commonly known as extinction and is visually represented in Fig (1), below. 


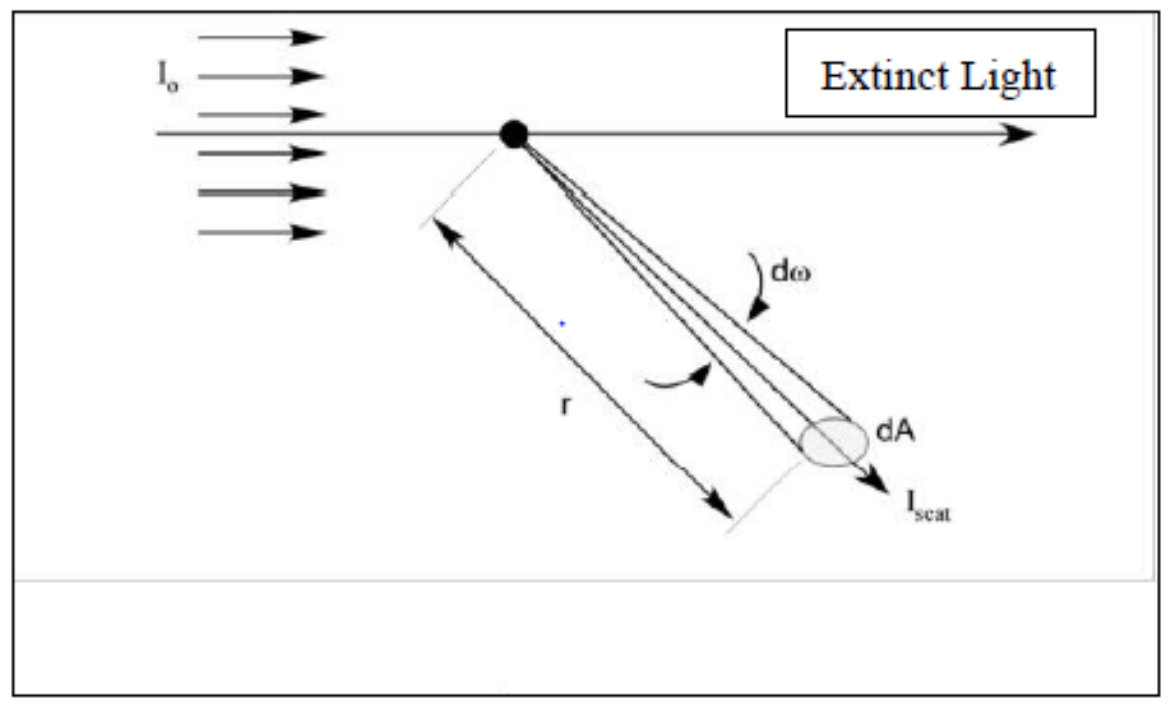

Figure 3: Interaction of light with a small particle describing the phenomenon of scattering and extinction.

The power of the resultant, lower intensity beam can be measured using photodetectors. This intensity loss is described by Beer's law [9],[13] given below:

$$
\tau_{i}=-\ln \left(\frac{I_{t}}{I_{o}}\right)=\frac{\pi}{4} C_{n} L \int_{0}^{\infty} Q\left(\pi D / \lambda_{i}, m\right) f(D) D^{2} d D, \quad \text { Equation } 1
$$

Where $\tau_{i}$ is the extinction or transmissivity at a given wavelength $\lambda_{i} ; I_{o}$ and $I_{t}$ are the intensities of incident and transmitted light at wavelength $\lambda_{i} ; C_{\mathrm{n}}$ is the average number density of the particles in the medium; $L=$ path length the light beam travels through the sample, $Q\left(\pi D / \lambda_{i}, m\right)$ is the extinction efficiency or extinction coefficient of a particle with diameter $\mathrm{D}$ and refractive index $\mathrm{m}$ at a given wavelength $\lambda_{\mathrm{i}} ; f(D)$ is the particle size distribution to be obtained which is assumed as a log normal function.

Equation 1can be categorized as a Fredholm equation of the first kind. Fredholm equations are integral equations where the kernel has integration limits as constants. In Eq. (1), the kernel is the extinction efficiency $Q\left(\pi D / \lambda_{i}, m\right)$. The determination of size distribution functions then 
reduces to a solution of Eq. (1) for a given $f(D)$ using extinction measurements performed at selected wavelengths. However this equation is ill conditioned due to the oscillatory behavior of the kernel function versus the particle size, namely the extinction coefficient $Q$ which depends on the ratio of the particle size to the wavelength of light incident (more commonly called the size parameter) [18].

In particle sizing, there are two types of model algorithms to solve these ill conditioned equations. The dependent model assumes the particles adhere to a known size distribution. Due to the lack of a fundamental mechanism or model to build particle size distribution functions theoretically, various size distributions functions have been used based on probability analysis or empirical observations [2], [14-17]. Among these functions, the log-normal distribution is most commonly used and is described below as it has been known to describe particle size distributions most accurately.

$$
f(D)=\frac{1}{\sqrt{2 \pi} D \ln (\sigma)} \exp \left[-\frac{1}{2(\ln \sigma)^{2}}\left(\ln D-\ln D_{\text {mean }}\right)^{2}\right] \quad \text { Equation } 2
$$

In the equation $\sigma$ represents the standard deviation of the size distribution and $D_{\text {mean }}$ is the mean diameter of the size distribution. The second model (independent model) does not assume any particle size distribution and solves Fredholm equations of the first kind directly.

When the size distribution function to be sought can be approximated by a known log-normal function, Eq. (1) can be modified into the equation described below by taking the ratio of intensity loss or transmissivities at two well-chosen wavelengths. This ratio yields the following expression:

$$
R_{i j}=\frac{\tau_{i}}{\tau_{j}}=\frac{\bar{Q}\left(\lambda_{i}, D_{32}\right)}{\bar{Q}\left(\lambda_{j}, D_{32}\right)} \quad \text { Equation } 3
$$

Where, 


$$
\begin{gathered}
\bar{Q}\left(\lambda_{i}, D_{32}\right)=\frac{\int_{0}^{\infty} Q\left(\frac{\pi D}{\lambda_{i}}, m\right) f(D) D^{2} d D}{\int_{0}^{\infty} f(D) D^{2} d D} \quad \text { Equation } 4 \\
D_{32}=\frac{\int_{0}^{\infty} f(D) D^{3} d D}{\int_{0}^{\infty} f(D) D^{2} d D} \quad \text { Equation } 5
\end{gathered}
$$

Where $R_{i j}$ is the ratio between measured extinction at wavelengths $\lambda_{i}$ and $\lambda_{j}$. $\bar{Q}$ and $D_{32}$ are the mean extinction coefficients and Sauter mean diameters defined in Eq. (4) and Eq. (5).

The extinction efficiency or extinction coefficient is a fundamental parameter that plays an important role in the interaction of a particle and an incident light wave. Formally, the extinction efficiency is defined as a ratio of the total extinction cross section (area of the wave front acted on by the particle) to the geometric cross section of the particle and is expressed below as:

$$
Q_{\text {ext }}=\frac{4 \times C_{\text {ext }}}{\pi \times D^{2}} \quad \text { Equation } 6
$$

Where $\mathrm{C}_{\text {ext }}$ is the total extinction cross section and $\mathrm{Q}_{\mathrm{ext}}$ is the extinction efficiency. For a sphere like polystyrene, the total extinction cross section $\left(\mathrm{C}_{\mathrm{ext}}\right)$ is $(\pi / 4) D^{2}$. An important aspect of extinction is that the ratio of particle size to the incident light wavelength (called the size parameter) is of more importance rather than the absolute value of either. Hence $Q_{\text {ext }}$ may also be defined as the fraction of cross sectional area of the particle that acts on the incident wave front. Particles that are very small relative to the wavelength of light incident on them are inefficient in extinction $($ Qext $<<1)$ but, the extinction efficiency rises rapidly as the fourth power of particle size to where, at sizes equal to or greater than the incident light wavelength

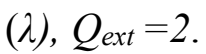


The behavior of the average extinction efficiency coefficient defined in Eq. (4) is shown in Fig. (4), for varying standard deviation values, at wavelengths of $450 \mathrm{~nm}$ and $7000 \mathrm{~nm}$ for polystyrene particles with diameters between 1-10 $\mu \mathrm{m}$. At the shorter wavelength of $450 \mathrm{~nm}$, fine ripple structures and a higher ambiguity exist in average extinction efficiency $(\bar{Q})$ values for different standard deviation values. On the other hand, the average extinction efficiency $(\bar{Q})$ values using the $7000 \mathrm{~nm}$ laser show no such ambiguity to changes in standard deviation values. Also it is important to note that the average extinction efficiency values tend towards a value of 2 when the ratio of the particle size to wavelength of light incident (the size parameter) increases. This is demonstrated in the case of the $450 \mathrm{~nm}$ laser at particles greater than $4 \mu \mathrm{m}$.

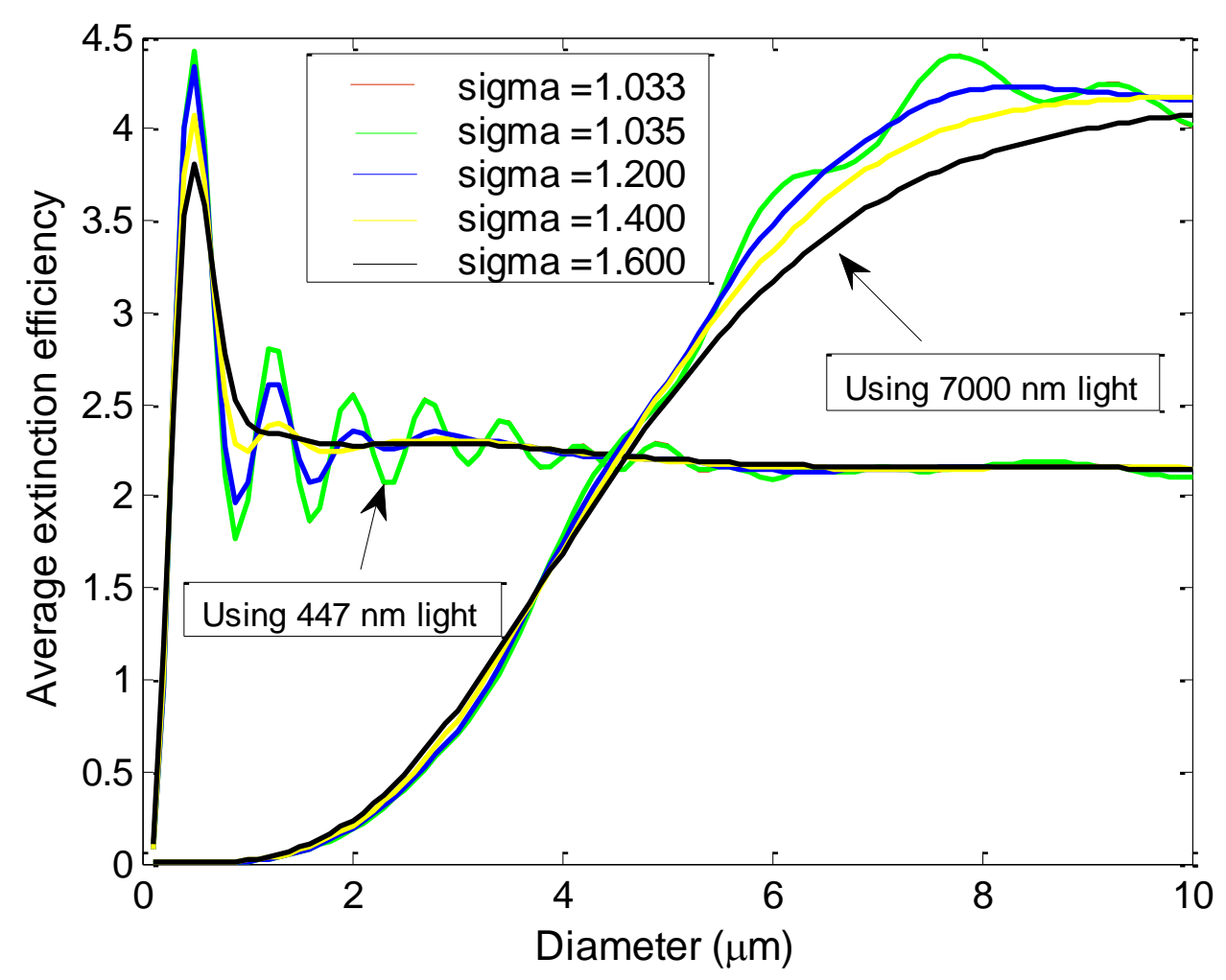

Figure 4: $\overline{\mathbf{Q}}$ at two distinct wavelengths vs diameter for increasing distribution widths (indicated in the direction of arrows). Refractive index is taken as $m=1.6143$ at $\lambda=450 \mathrm{~nm}$ and $m=1.5633$ at $\lambda=7000 \mathrm{~nm}$ for the polystyrene particles. 
In order to obtain particle diameters, a relatively simple method was developed by Ma et al. to invert diameter data from average extinction coefficient values [9]. By observing Fig. (4), it can be stated that the extinction efficiencies obtained using the $450 \mathrm{~nm}$ laser is independent of standard deviation beyond a diameter of $3 \mu \mathrm{m}$. The average extinction efficiencies are also independent of standard deviations for particle sizes up to $5.5 \mu \mathrm{m}$ using the $7000 \mathrm{~nm}$ laser. To obtain information on diameter of particles, the ratio of extinction efficiencies can be taken at these two wavelengths [9]. This results in a relationship between measured transmissivities versus diameters described by Eq. (3) discussed earlier and is shown graphically in Fig. (5), below. For example, a ratio of 1 will result in a diameter prediction of $4.4 \mu \mathrm{m}$. As seen in Fig. (5), dependence of the ratio of extinction efficiencies on standard deviation is more pronounced at larger diameters. This ambiguity could be mitigated by using a source of light with a wavelength much greater than the particle size resulting in a size parameter lesser than 1. However these light sources will be highly sensitive to black body radiation even at local room temperatures (as described by Weins displacement law $\lambda T=b$ where $\lambda$ is the wavelength of light; $T$ is the black body temperature; $b$ is a constant whose value is approximately equal to $2.897 \times 10^{-3} \mathrm{mK}$ ) and will not be practical due to interference signals arising from surrounding radiation that will affect the measurement and induce errors in resultant diameter and number density values.

In order to obtain a size distribution function, both diameters and standard deviations are required. Hence it is important to predict standard deviation values once diameters are obtained. Standard deviation may be predicted when the ratio of extinction efficiencies show a dependence on the distribution width of particles being sampled. Utilizing Fig. (5), above assume the sample particle diameter to be $8 \mu \mathrm{m}$ with an extinction efficiency ratio of 1.88 . The standard deviation will be obtained by expressing the ratio of extinction efficiency as a function of different distribution widths for the diameter $(8 \mu \mathrm{m})$ predicted. 


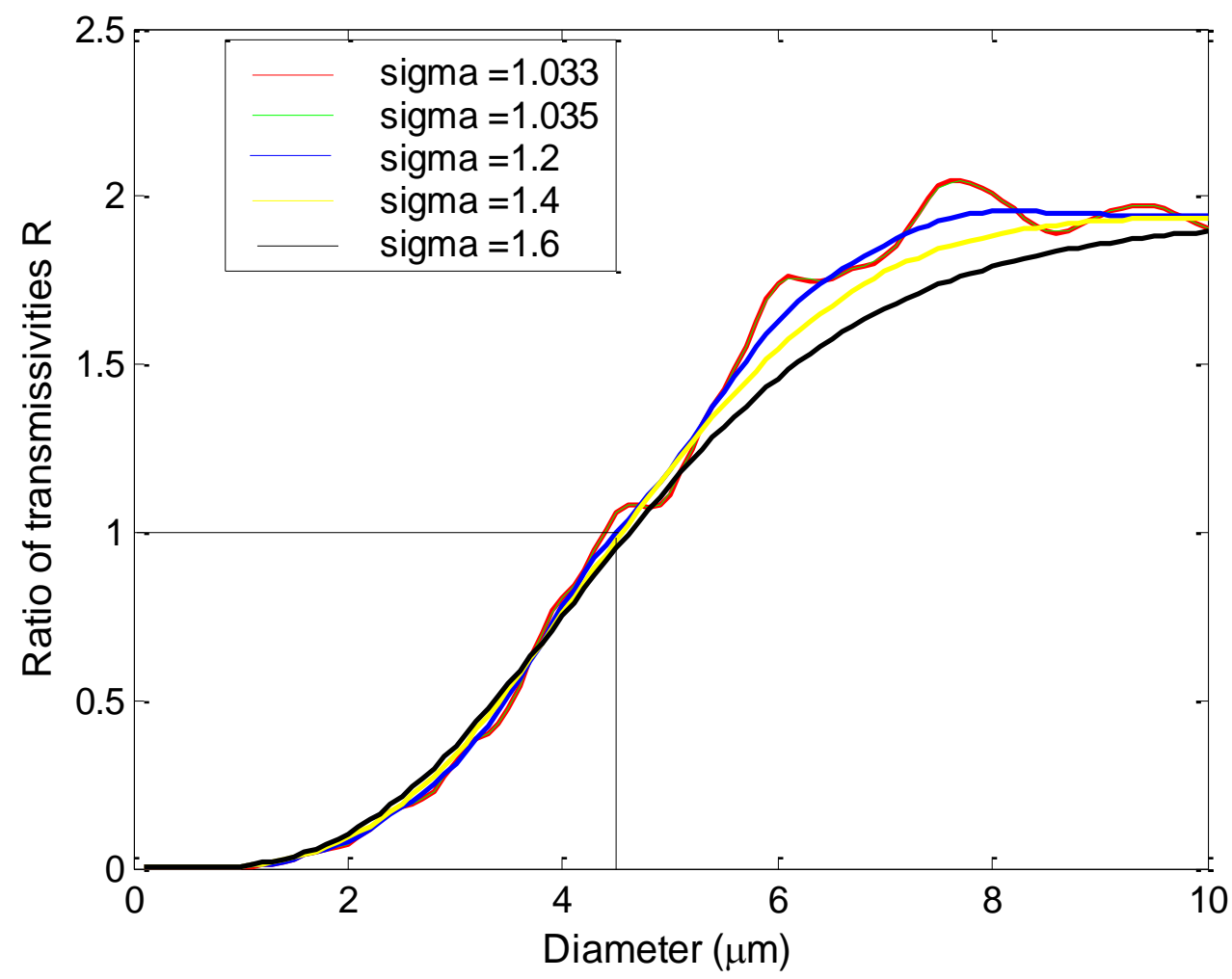

Figure 5: Ratio of $\bar{Q}$ using $450 \mathrm{~nm}$ and $7000 \mathrm{~nm}$ lasers at 5 distribution widths (standard deviations) using polystyrene particles. Note that ripple structure fluctuations of average extinction efficiencies occur at distribution widths close to 1.

This creates a relationship between the two parameters and is shown graphically in Fig. (6). It should be noted that in order to make measurements for diameters, standard deviations and concentrations, a minimum of three lasers would be required to provide information on these parameters over the entire size range as no information on standard deviation is available for diameters between 1- $6 \mu \mathrm{m}$ as seen by Fig. (5). Two lasers are shown in the measurement concept for ease of analysis.

As seen in Fig. (6), the ratio R varies non-monotonically for diameters which show dependence on the distribution width. The quantity $S_{\sigma}$ helps in recognizing the non-monotonic behavior of the ratio vs distribution width described by Ma et al[9]: 


$$
S_{\sigma}=\operatorname{abs}\left(\frac{\frac{d R}{R}}{\frac{d D_{32}}{D_{32}}}\right)
$$

\section{Equation 7}

Where $R$ is the ratio of extinction efficiencies; $D_{32}$ is the sauter mean diameter. Once diameters and distribution widths have been obtained, the diameter of a log-normal distribution is related to the size distribution function by the following relation:

$$
\ln \left(D_{\text {mean }}\right)=\ln \left(D_{32}\right)-\frac{5}{2}(\ln \sigma)^{2}
$$

\section{Equation 8}

Hence $D_{\text {mean }}$ is obtained after diameters and standard deviations have been obtained and so is the log-normal distribution function.

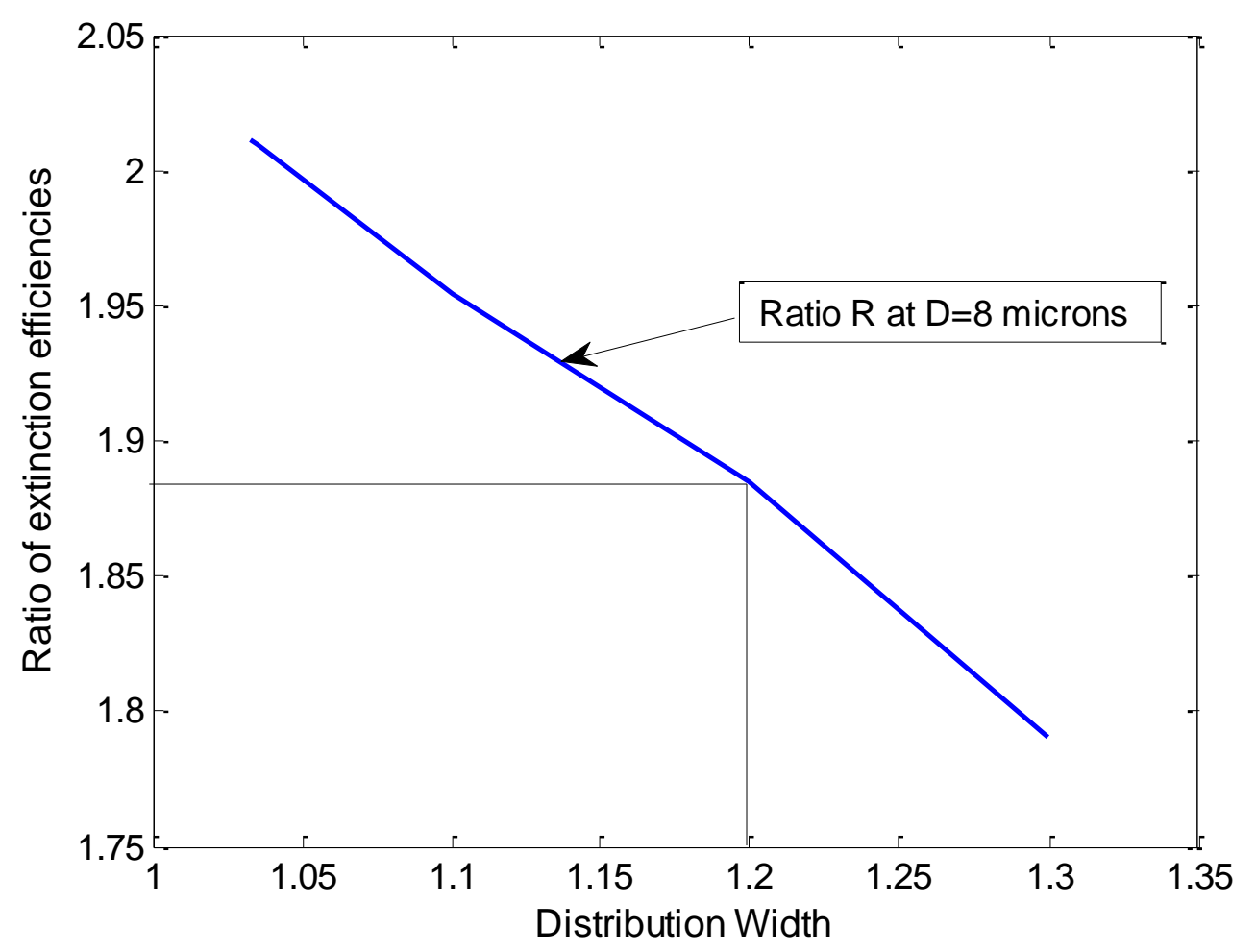

Figure 6: Variation of $R$ versus distribution width for a diameter of $8 \mu \mathrm{m}$ using a combination of $450 \mathrm{~nm}$ and $7000 \mathrm{~nm}$ lasers resulting in a distribution width prediction of 1.2. 


\section{PROOF OF CONCEPT EXPERIMENT 1: STATIC SETUP}

\section{Brownout Landings and Sand Particles Entrained}

Aircraft performing desert landings frequently experience reduced visibility due to airborne sand and dust entrained from the desert floor by rotor downwash [22]. These low visibility conditions (more commonly called brownout) can result in loss of aircraft components and in most severe cases, loss of life. It becomes important to characterize the diameters and number densities of the sand particles to design an experiment that simulates brownout conditions accurately. In an effort to provide pilots better situational information to make brownout landings safer, research was previously carried out to characterize particulate matter in brownout conditions using four aircraft in the La Posa drop zone of the Yuma Proving Grounds. High volumes of air were sampled using a cyclone pre-separator with and without cascade impactors [22]. Results of the sampling are summarized in Table. 1 below for four different aircraft utilized in military operations.

Table 1: Particle characterization of a brownout cloud for different airframes. Source, Defense Advanced Research Projects Agency (DOD) Strategic Technology Office. Issued by U.S. Army Aviation and Missile Command Under Contract No. W31P4Q-07-C-0215.

\begin{tabular}{|l|c|c|c|c|}
\hline \multirow{2}{*}{$\begin{array}{l}\text { Particle Size } \\
\text { Range }(\mu \mathrm{m})\end{array}$} & \multicolumn{4}{|c|}{ Concentration $\left(\mathrm{N}_{\mathrm{\rho}} / \mathrm{m}^{3}\right)$} \\
\cline { 2 - 5 } & Aircraft 1 & Aircraft 2 & Aircraft 3 & Aircraft 4 \\
\hline$<10$ & $1.76 \times 10^{12}$ & $2.12 \times 10^{12}$ & $4.85 \times 10^{11}$ & $6.57 \times 10^{11}$ \\
$13-60$ & $5.94 \times 10^{9}$ & $5.66 \times 10^{9}$ & $1.26 \times 10^{9}$ & $5.44 \times 10^{9}$ \\
$60-125$ & $6.19 \times 10^{7}$ & $2.29 \times 10^{8}$ & $1.69 \times 10^{8}$ & $5.41 \times 10^{8}$ \\
$125-250$ & $3.32 \times 10^{6}$ & $7.54 \times 10^{6}$ & $1.31 \times 10^{7}$ & $1.45 \times 10^{7}$ \\
$250-350$ & $1.31 \times 10^{6}$ & $1.34 \times 10^{6}$ & $3.88 \times 10^{6}$ & $1.91 \times 10^{6}$ \\
$>350$ & 0 & $1.76 \times 10^{5}$ & $7.04 \times 10^{5}$ & $2.63 \times 10^{5}$ \\
\hline
\end{tabular}


Where $\mathrm{N}_{\mathrm{p}}$ is the number of particles. On observing particle concentrations in the table, it may be noted that number densities of particles in the size range of less than $10 \mu \mathrm{m}$ dominate larger particle number densities in the brownout cloud by a minimum of 2 orders of magnitude. These submicron particles can easily deposit on turbine blades and deteriorate blade performance by altering the blade geometry and blocking cooling paths. Hence, the focus of the experimentation was to test particles in the $1-10 \mu \mathrm{m}$ size range and validate the ability of the technique to provide size and concentration information for these particles.

\section{Experimental Setup and Instrumentation}

In order to resolve diameters and concentrations, a static experiment was designed consisting of polystyrene latex spheres (purchased from Magsphere Inc.) in a water dispersion. The water medium was utilized to create a suspension of particles in varying concentrations through which laser light could be passed. A static experiment was setup in order to determine the validity of the technique employed at the most fundamental level. Future work entails the development of flow setups using the same measurement technique to simulate engine inlet conditions more realistically. Polystyrene spheres were chosen as test particles on account of their ease of calibration, readily available refractive index and their spherical geometry. The refractive index of the particles were obtained from literature at selected wavelengths for $20^{\circ} \mathrm{C}$ [23]. It has been noted that a change in the imaginary component of refractive index does not impact the average extinction efficiency values greatly and hence the imaginary component of the refractive index is not considered in the analysis. The sizes of test particles were $1.32 \mu \mathrm{m}$ at $6.6 \%$ solids, $3.9 \mu \mathrm{m}$ at $10 \%$ solids and $5.1 \mu \mathrm{m}$ at $10 \%$ solids respectively as obtained from the manufacturer. Particle standard deviations were $1.1,1.033$ and 1.035 for $1.32 \mu \mathrm{m}, 3.9 \mu \mathrm{m}$ and $5.1 \mu \mathrm{m}$ particles respectively as provided by manufacturer data. Test particle solutions were diluted to the desired level of concentrations comparable to those experienced by aircraft in 
degraded visual environments described in Table 1 above. The concentration range of sampled polystyrene particles is summarized in Table 2 below.

Table 2: Maximum and minimum concentration measured for the particles sampled in the static setup.

\begin{tabular}{|c|c|c|c|}
\hline \multirow{2}{*}{$\begin{array}{c}\text { Sample } \\
\text { Concentration } \\
\left(\mathrm{Np} / \mathrm{m}^{3}\right)\end{array}$} & 1.32 & 3.9 & 5.1 \\
\cline { 2 - 4 } & \multicolumn{3}{|c|}{ Diameter $(\mu \mathrm{m})$} \\
\hline $\begin{array}{c}\text { Minimum } \\
\text { Maximum }\end{array}$ & $\begin{array}{c}1 \times 10^{13} \\
8.33 \times 10^{11}\end{array}$ & $\begin{array}{c}4.28 \times 10^{12} \\
7.82 \times 10^{11}\end{array}$ & $2.25 \times 10^{12}$ \\
\hline
\end{tabular}

Where $\mathrm{N}_{\mathrm{p}}$ is the number of particles. It is important to note the decrease in the upper limit of detection for the maximum concentration as the particle size increases. This can be attributed to detection at the noise floor level of the detector resulting in erroneous measurements due to greater extinction. The instrumentation setup employed consisted of two continuous wavelength laser sources, a $447 \pm 5 \mathrm{~nm}$ (Dragon laser) with a power output of 500 milliwatts and a $635 \mathrm{~nm} \pm 10 \mathrm{~nm}$ (Thorlabs) handheld fiber- coupled laser with a power output of 2.5 milliwatts. The $447 \mathrm{~nm}$ laser had a TE00 transverse mode with a beam divergence of less than 1.5 milliradian and a beam diameter of $5 \mathrm{~mm}$. The sensor used to measure intensity of $447 \mathrm{~nm}$ light was a photodectector (Thorlabs PDA-25K ) that had an operating wavelength range of $150-550 \mathrm{~nm}$ and a rise time of 46 nanoseconds along with a noise equivalent power in the range of $7 \times 10^{-12}-3 \times 10^{-10}\left(\mathrm{~W} / \mathrm{H}_{\mathrm{z}}{ }^{1 / 2}\right)$. The maximum responsivity of this detector was $0.118(\mathrm{~A} / \mathrm{W})$ at $430 \mathrm{~nm}$ and the active area of the detector was $4.8 \mathrm{~mm}^{2}$. In order to focus the $447 \mathrm{~nm}$ laser light onto the detector, a combination of plano convex and plano concave lenses with focal lengths of $f=+100 \mathrm{~mm}$ and $f=-50 \mathrm{~mm}$ respectively were used. These two lenses were separated by a distance of $50 \mathrm{~mm}$. The $635 \mathrm{~nm}$ fiber coupled laser used a aspheric fiber port (Thorlabs PAFX-7-A) for collimation of the laser beam. The collimated laser beam had a divergence of 0.467 
milliradians. The sensor used to measure intensity of $635 \mathrm{~nm}$ light was a photodetector (Newport 918D-SL-OD3R) that had an operating wavelength range of $400-1100 \mathrm{~nm}$ and a rise time of less than 2 microseconds. The detector had an active area of $1 \mathrm{~cm}^{2}$ and a maximum responsivity of $0.5(\mathrm{~A} / \mathrm{W})$ at a wavelength of $950 \mathrm{~nm}$. The output from these two detectors was then processed using a data acquisition system (National Instruments USB 6351). This multifunction DAQ was utilized at a sampling rate of 1 Megasamples per second. The acquisition system had a 16-bit resolution and a maximum input voltage of $10 \mathrm{~V}$. Extinction of laser light due to the presence of water to disperse the particles and container to hold the sample was taken into account by measurements without the particles in the suspension. The test schematic is shown below in Fig. (7).

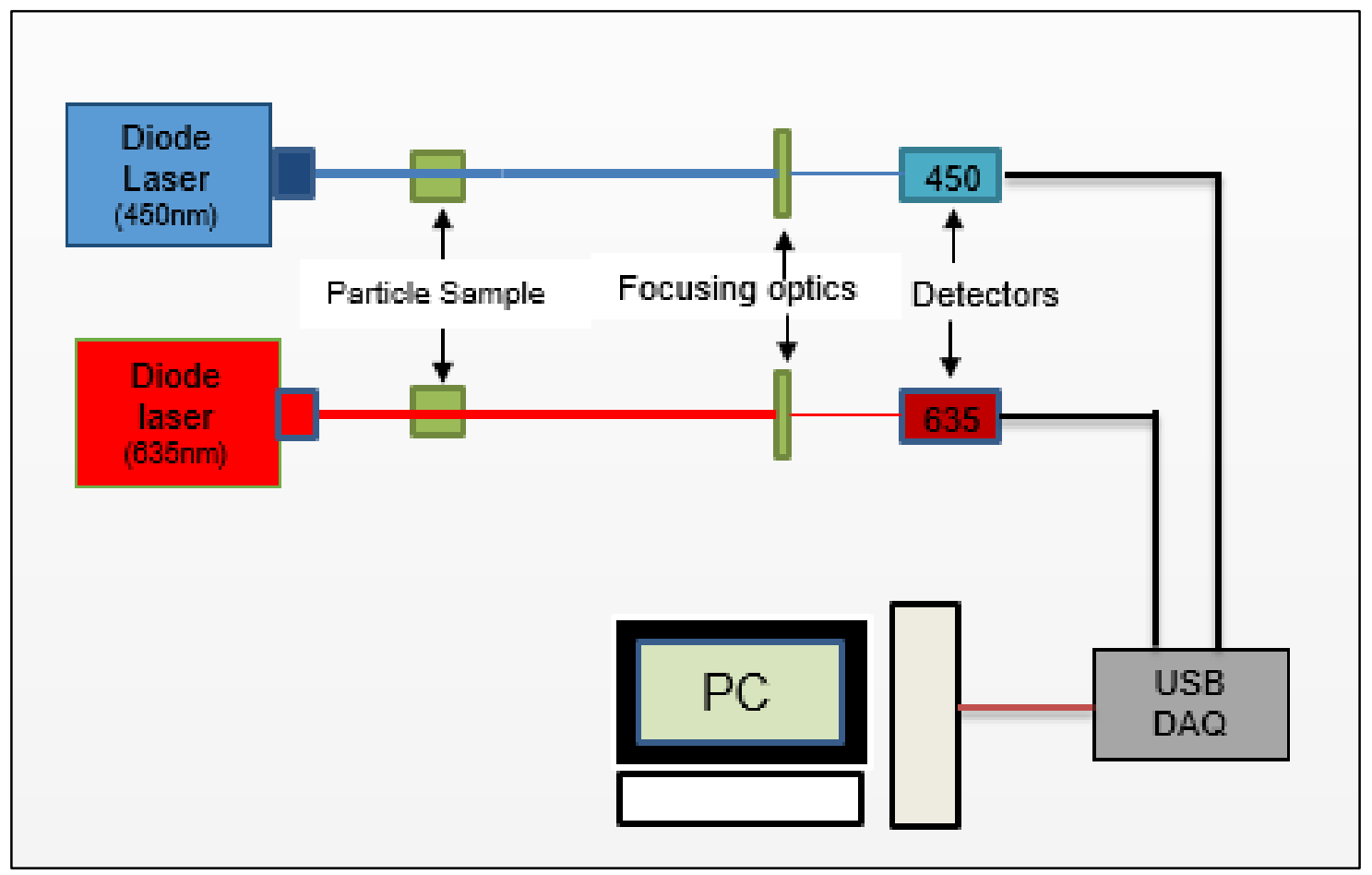

Figure 7: Schematic representation of static experimental setup.

The output voltage from the photodiodes was converted into power using a LabVIEW virtual interface that converts the measured voltage into power while acquiring data. The values of 
power obtained using the two photodiodes was validated using an optical power meter. In order to obtain transmissivities as given by Eq. (1), power of the laser beam was measured with and without the presence of particles in the beam path. The ratio of the incident light intensity without the particles $\left(I_{o}\right)$ to the transmitted light intensity in the presence of the sample $\left(I_{t}\right)$ was then recorded assuming the beam waist was constant for the two laser light sources. This allows the following assumption for a given wavelength source:

$$
\frac{I_{t}}{I o}=\frac{P_{t}}{P_{o}}=\tau_{i} \quad \text { Equation } 9
$$

Where $P_{t}$ and $P_{o}$ are the transmitted and incident power on the photodiodes and $\tau_{\mathrm{i}}$ is the transmissivity at wavelength $\lambda_{\mathrm{i}}$. The resultant ratio of transmissivities $(\mathrm{R})$ was obtained by taking the ratio of the two individual transmissivities $(\tau)$ obtained experimentally at the two wavelengths of light $(447 \mathrm{~nm}$ and $635 \mathrm{~nm})$. The mean particle diameter was obtained by inferring particle diameters from the ratio $(\mathrm{R})$ using the functional relationship between diameters and average extinction efficiency as expressed by Eq. (3) and graphically represented in Fig. (5). The testing procedure follows the process as described by Fig. (8), below.

Illuminate test section with two light sources

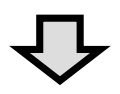

Measure light intensity loss due to particles

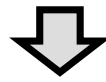

Obtain ratios $(\mathrm{R})$ of intensity loss at the two selected wavelengths

Use $\mathrm{R}$ vs diameter plots and obtain measured diameters

Figure 8: Flow chart illustrating the measurement technique utilized. 
Repeatability of tests was ensured by multiple sample testing over 24 hours to obtain a constant transmission of power from both photodiodes using both light sources. Two sets of measurements were performed independently on the three test particle sizes within the concentration limits as described in Table 2.

\section{Results from the Static Setup (Mono-dispersed Samples)}

The particles utilized in the sizing analysis were well characterized by the manufacturer using a Scanning Electron Microscope (SEM). This predetermined size would provide a check on the results obtained from the experiments. As described in the previous sections, the ratio of extinction efficiencies or transmissvities at two selected wavelengths was utilized to obtain information on diameters of particles in the dispersion. This ratio of tranmissivities vs. diameter shows an oscillatory behavior for the ratio $\mathrm{R}$ values obtained experimentally using the two light sources as seen in Fig. (A1), in the appendix. This oscillatory behavior points to multiple diameters possible for a given $\mathrm{R}$ value and can lead to ambiguity in the measurements. This ambiguity can be mitigated by increasing the spectral width $\left(\lambda_{i}-\lambda_{j}\right)$ of the two light sources where $\lambda_{\mathrm{i}}$ and $\lambda_{\mathrm{j}}$ are the two wavelengths of light used. The effect of this increase is shown in Appendix A where the number of possible diameters reduces to a unique value with an increase in the spectral width. Such a procedure was carried out to verify if the diameter measured was the actual diameter of the particles as measured by the SEM. On completion of the disambiguation, diameter values were recorded against the concentration range of measurements and are represented below. 


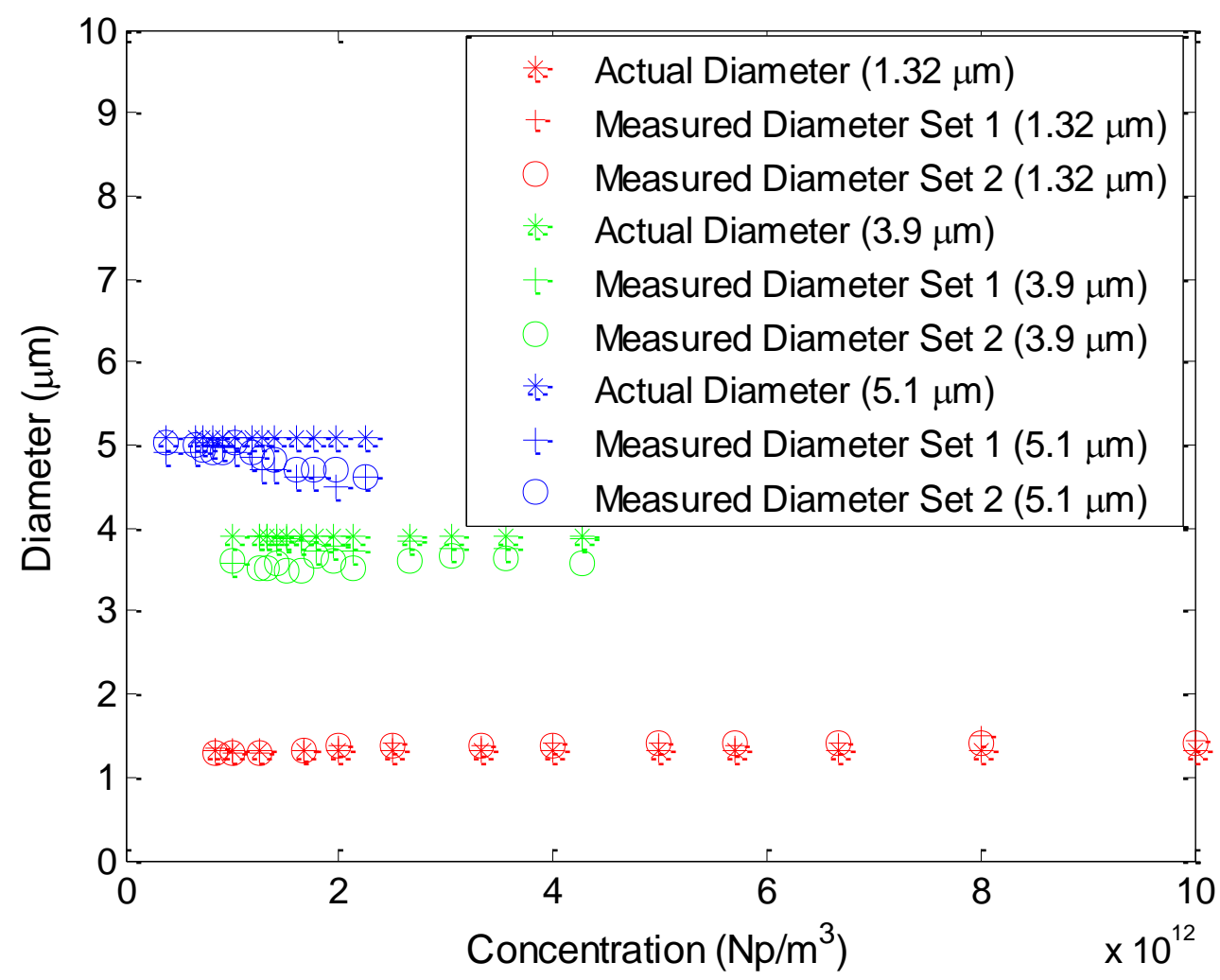

Figure 9: Diameter vs concentration measured compared with actual values for sampled polystyrene particles.

As seen from the figure above, the measurement technique predicts the diameter of the test particles with high accuracy. The maximum deviation in diameter measurements of all three particle sizes was $0.16 \mu \mathrm{m}$ which corresponds to a maximum relative error of $11 \%$ across all three test particle sizes sampled. The deviation of diameter at higher concentrations with the $5.1 \mu \mathrm{m}$ sample could be attributed to operation near the noise floor of the detector. Noise floor operations result from a weak incident signal on the detector due to high sample concentrations. On the other hand, the deviation at low concentrations for the $3.9 \mu \mathrm{m}$ sample can be attributed to the lack of a uniform presence of particles in the path of the laser beam. This results in a prediction of a diameter lower than the actual value due to a stronger signal incident on the detector and leads to errors in the measurement as the transmissivity $\left(\tau_{i}\right)$ is directly related to particle concentration and size. Another possible source of deviation could be the narrow 
spectral gap (the difference of wavelength between the two laser light sources used). This could result in the diameter measurements becoming very sensitive to the attenuation or transmissivity of light at the two wavelength sources as shown graphically in Fig. (A1) and consequently lead to errors in measurements.

Once the information on diameters was obtained, concentrations of particle samples were predicted using Beer's Law as shown in Eq. (1). Utilizing the information on transmissivity from data obtained using the two light sources, concentration values were calculated and are shown in Figs. (10-12). The concentration measurements show close agreement with respect to the actual concentration of the sample prepared. Once the diameters and concentrations were predicted, the next step in the sizing analysis was the prediction of the distribution width $\sigma$ in order to determine a particle size distribution. Utilizing the technique to determine standard deviations as described in the measurement concept section, the ratio $R$ obtained experimentally was compared with distribution widths ranging from 1-1.2 and were consequently predicted with a good degree of accuracy. As an example the standard deviation prediction for two independent measurements in $5.1 \mu \mathrm{m}$ samples is shown in Fig. (13). This standard deviation obtained was 1.05 for a predicted mean diameter of $4.86 \mu \mathrm{m}$ and 1.035 for a predicted mean diameter of $4.906 \mu \mathrm{m}$. The actual standard deviation as specified by the manufacturer was 1.035 . 


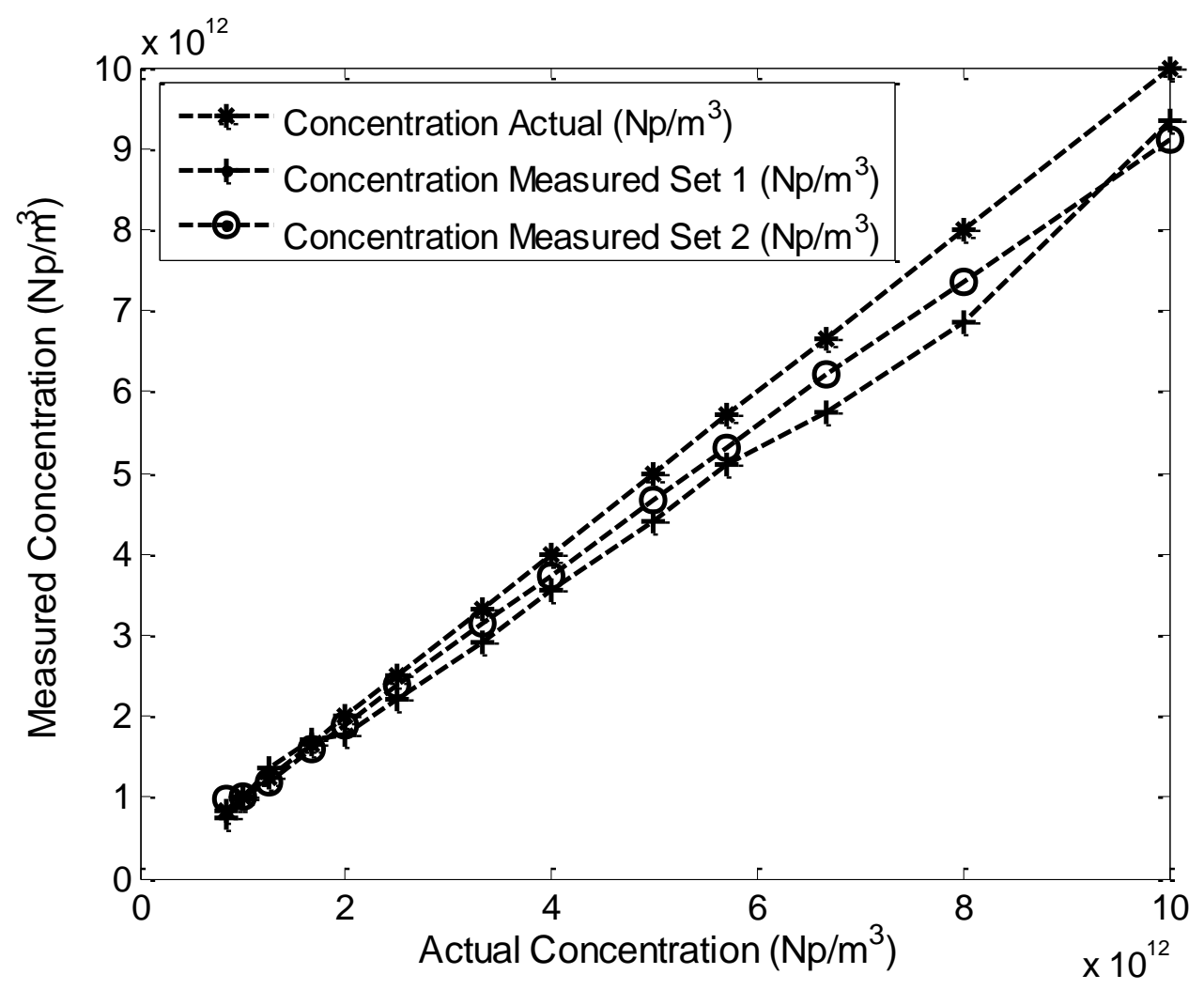

Figure 10: Concentration measured using $450 \mathrm{~nm}$ light for $1.32 \mu \mathrm{m}$ particles.

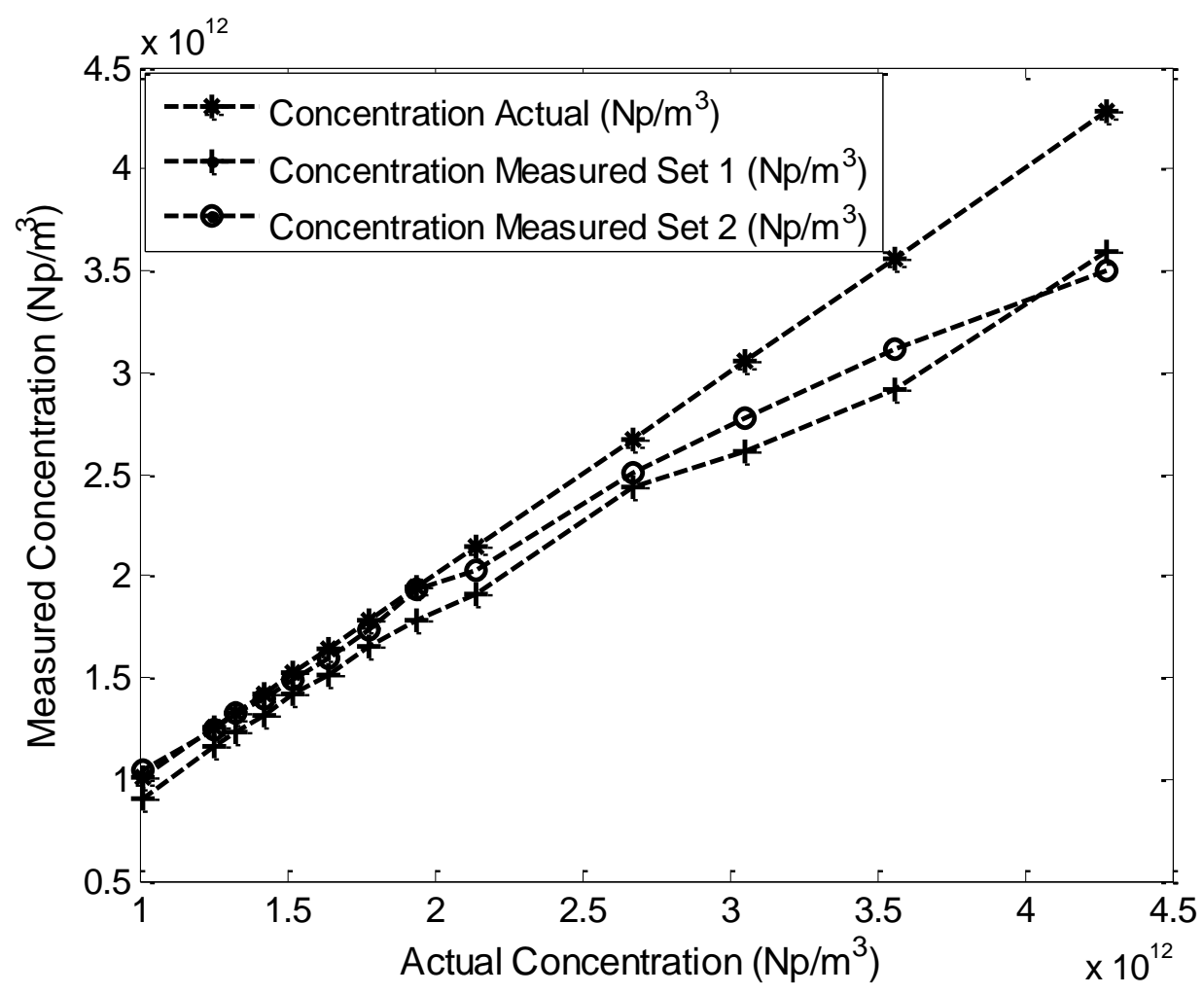

Figure 11: Concentration measured using $635 \mathrm{~nm}$ light for $3.9 \mu \mathrm{m}$ particles. 


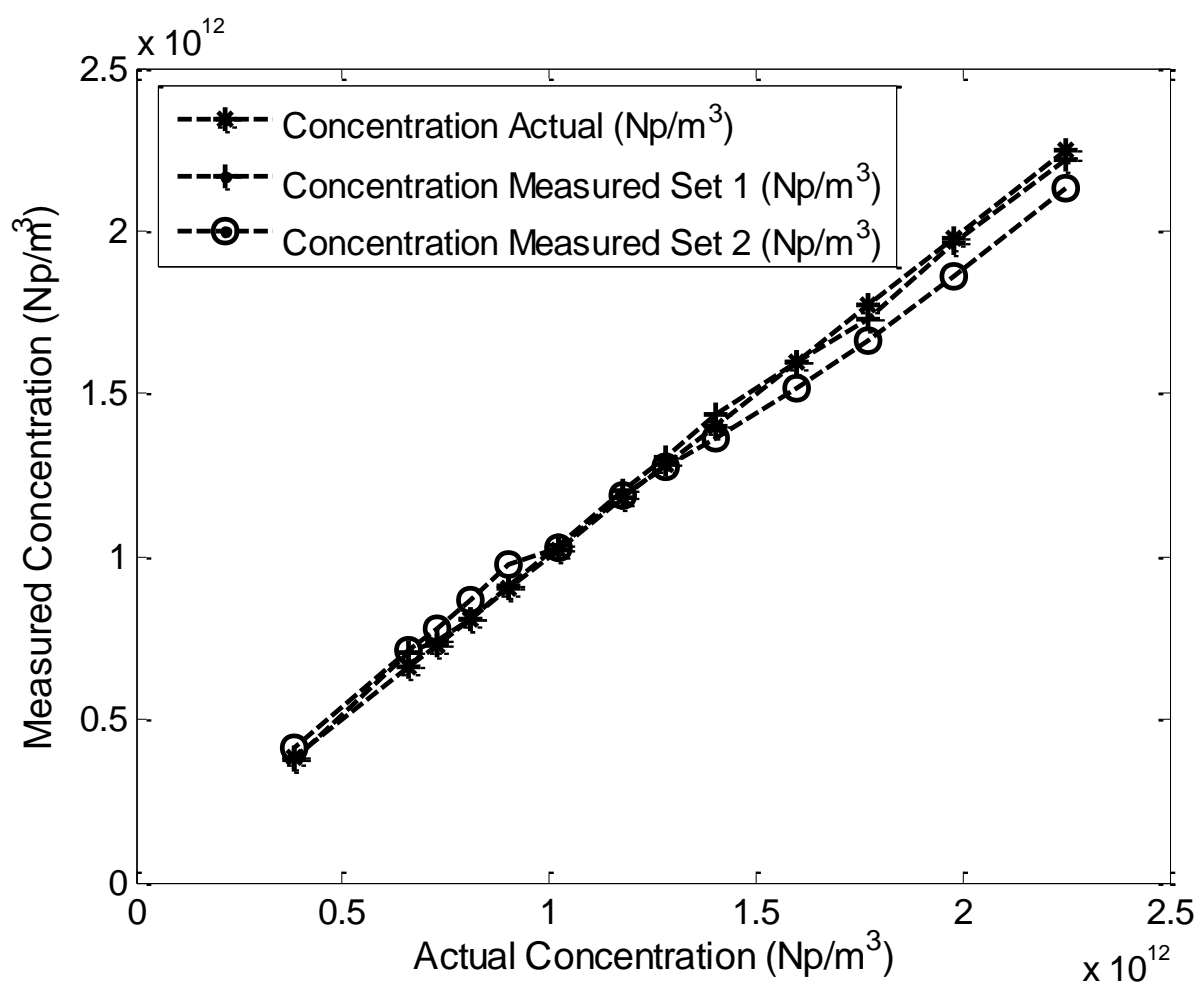

Figure 12: Concentration measured using $450 \mathrm{~nm}$ light for $5.1 \mu \mathrm{m}$ particles.

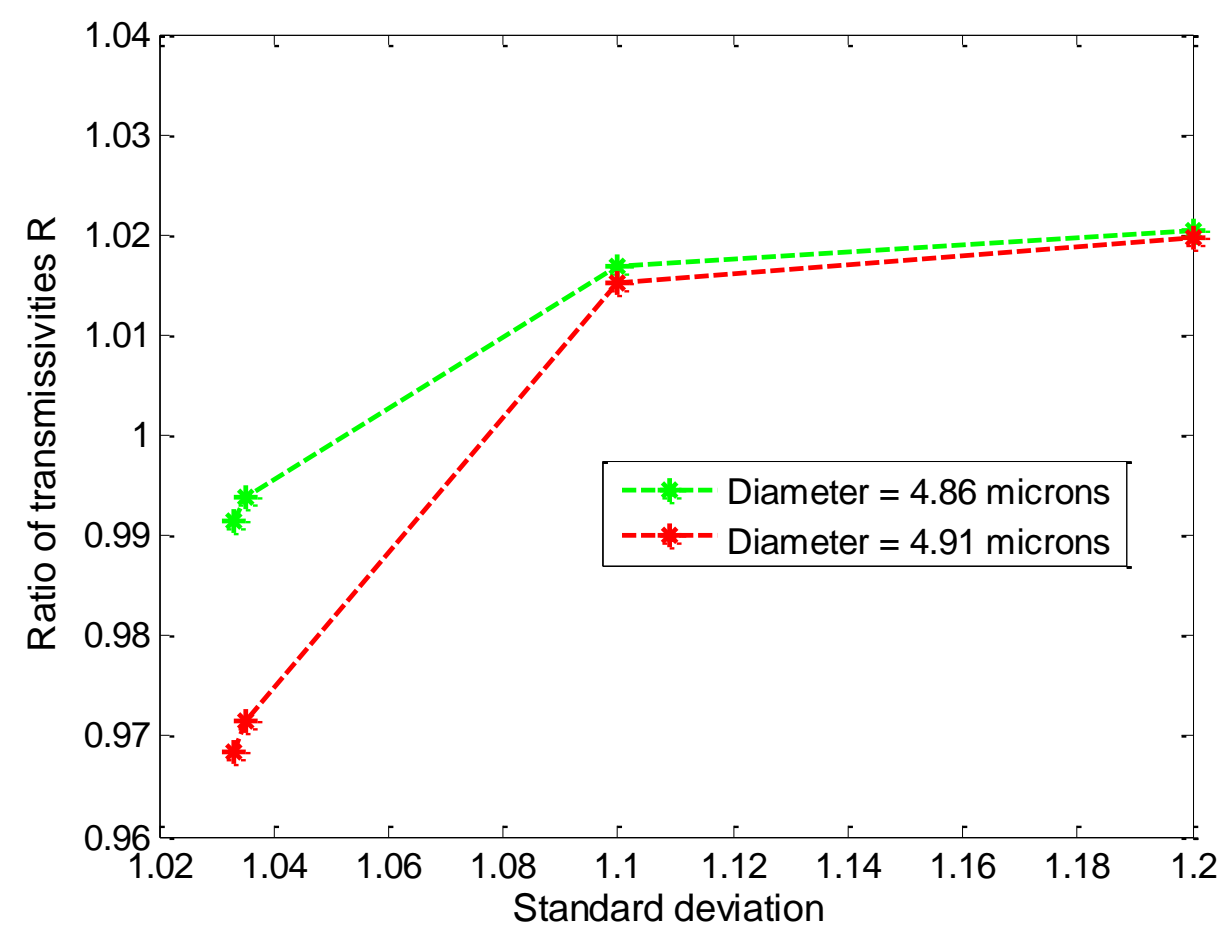

Figure 13: Standard deviation vs ratio of transmissivities for $5 \mu \mathrm{m}$ particles. 
Once the standard deviation and mean diameters were predicted, the next step was to create the log-normal particle size distribution function utilizing Eq. (2) and Eq. (8). Information on the actual diameter and standard deviations of particles was available from manufacturer data. The measured particle size distribution was obtained by utilizing predicted values of diameters and standard deviations. The resultant size distribution functions were recreated for the three test particles and are represented in Figs. (14-16), shown below. The diameters, concentration and size distributions obtained from the static dispersion of polystyrene particles in water show excellent conformance with the actual particle data. This validates the ability of the wavelength multiplexed laser extinction technique to determine size distributions of particles in a dispersion and is a fundamental step in the application of the technique to measure real time particle sizes, concentrations and standard deviations.

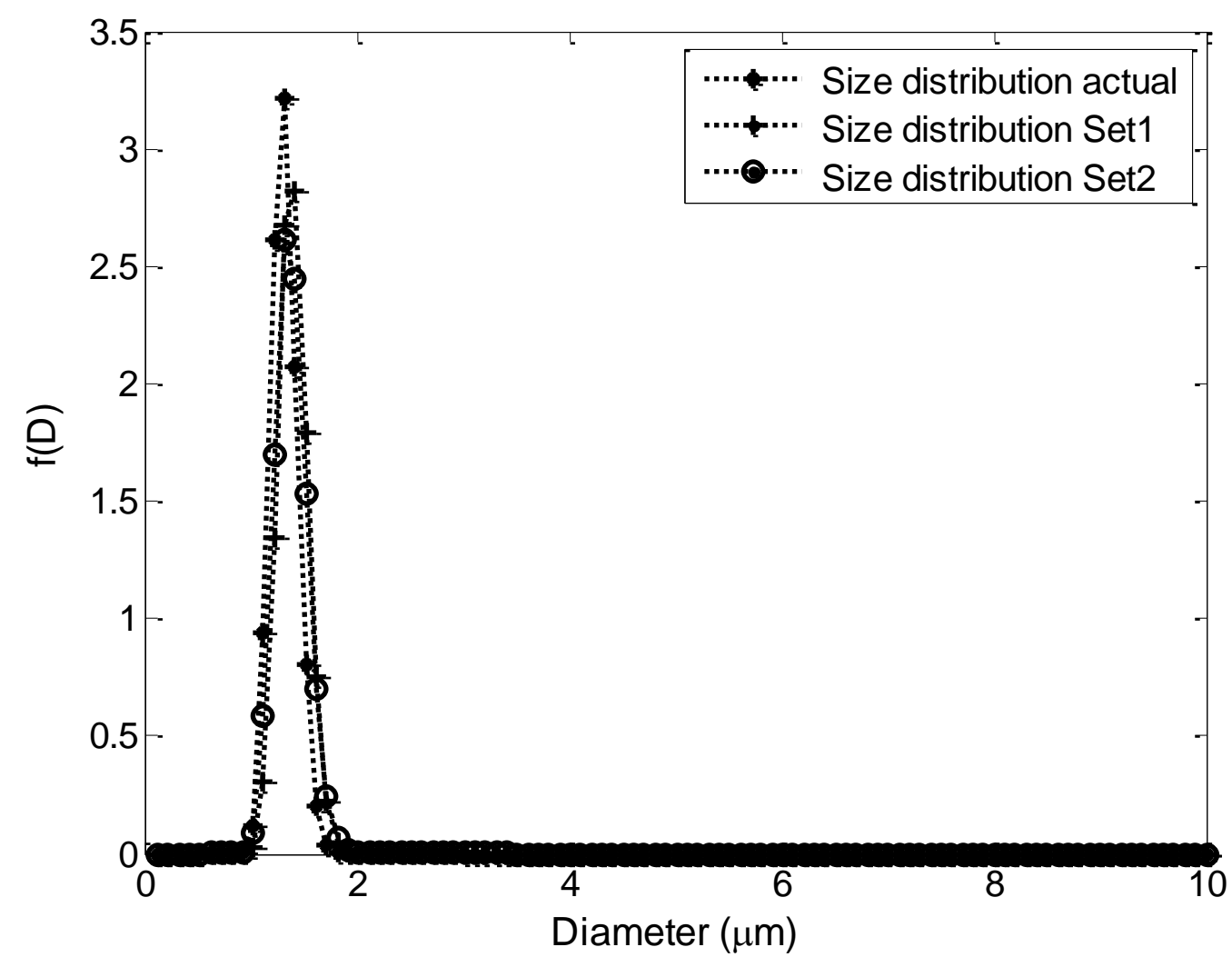

Figure 14: Particle size distribution for $1.32 \mu \mathrm{m}$ polystyrene latex. 


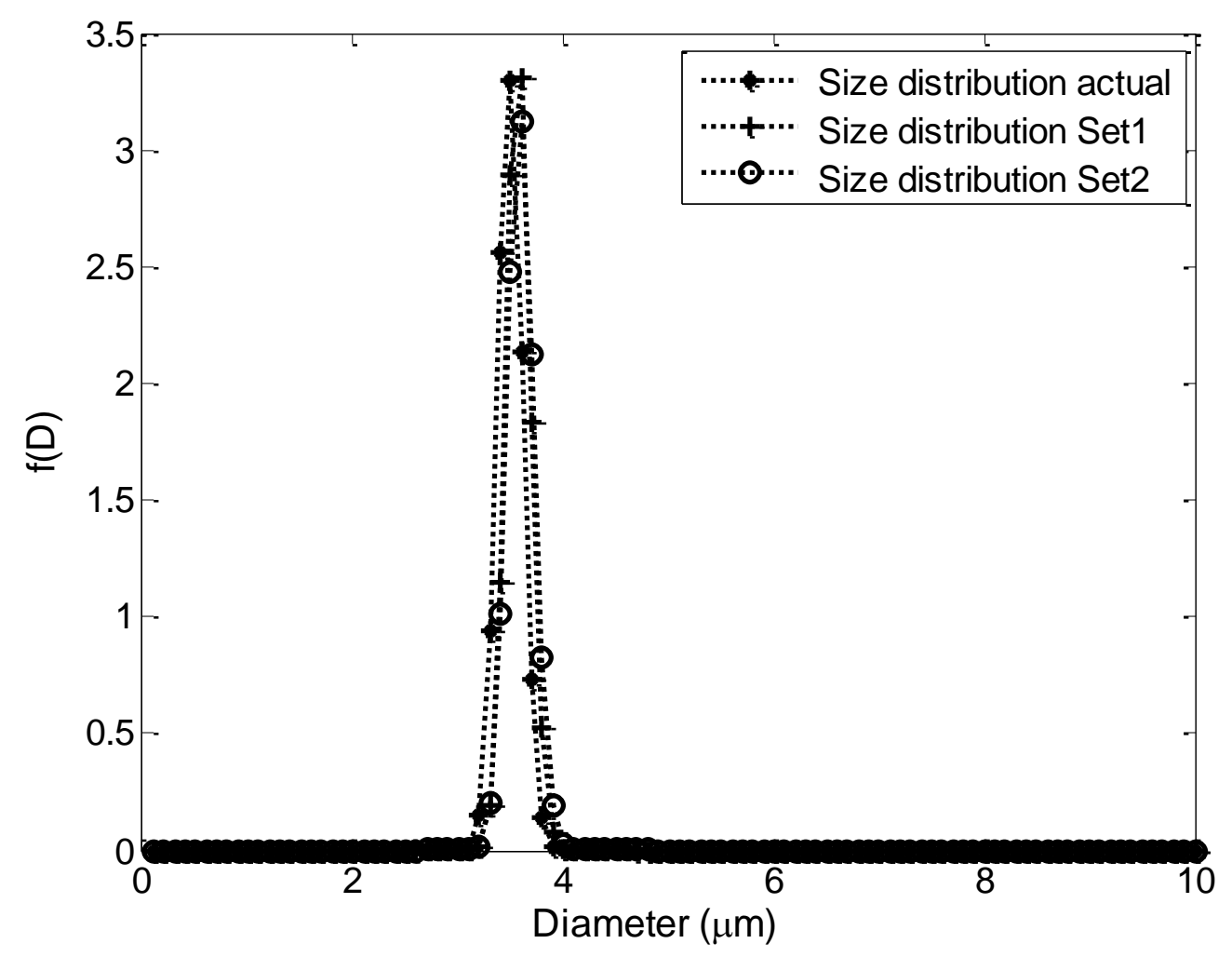

Figure 15: Particle size distributions for $3.9 \mu \mathrm{m}$ polystyrene latex.

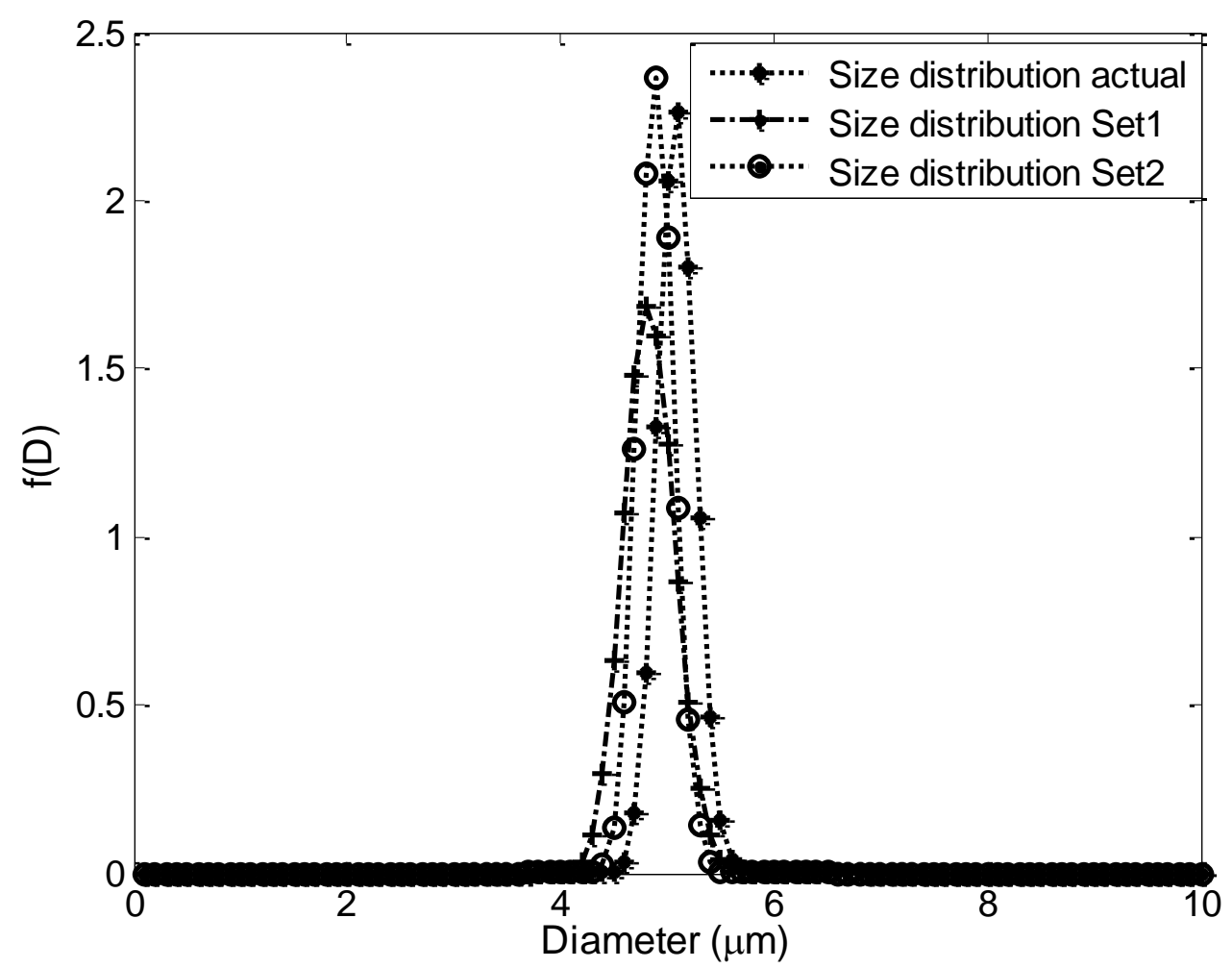

Figure 16: Particle size distributions for $5.1 \mu \mathrm{m}$ polystyrene latex. 


\section{Measurement of Diameters and Concentrations in a Poly-dispersed Sample}

This far the results reported were for mono-dispersed samples. It is important that the technique be able to resolve a mean diameter from a poly-dispersed sample of polystyrene in varying concentrations. This section will describe the results obtained from two separate poly-dispersed samples of the same polystyrene particles prepared using a mixture of $(3.9 \mu \mathrm{m} 5.1 \mu \mathrm{m})$ particles in varying concentrations and volumes using the relation for a weighted Sauter mean diameter:

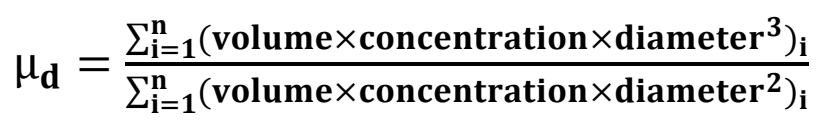

\section{Equation 10}

Where $\mathrm{n}$ is the number of particle samples of different sizes used to create the poly-dispersed sample and $\mu_{d}$ is the mean diameter of the polydispersed sample prepared. This statistical mean diameter would provide a validation check on the results obtained from the extinction measurements. The two batches prepared had a mean diameter of $4.3 \mu \mathrm{m}$ and $3.38 \mu \mathrm{m}$. Utilizing the experimental procedure described above to size mono-dispersed samples, mean diameters and concentrations were obtained for the poly-dispersed samples prepared and are shown in Figs. (17-19). As seen from the figures below, diameters and concentrations of the mixture were predicted in close conformance with the actual values. The maximum deviation of diameter values is $0.3 \mu \mathrm{m}$ and is obtained at lower concentrations. This result again could be attributed to a lack of uniform particle presence in the beam path resulting in lower diameter predictions than the actual values due to a stronger signal incident on the photodiodes. 


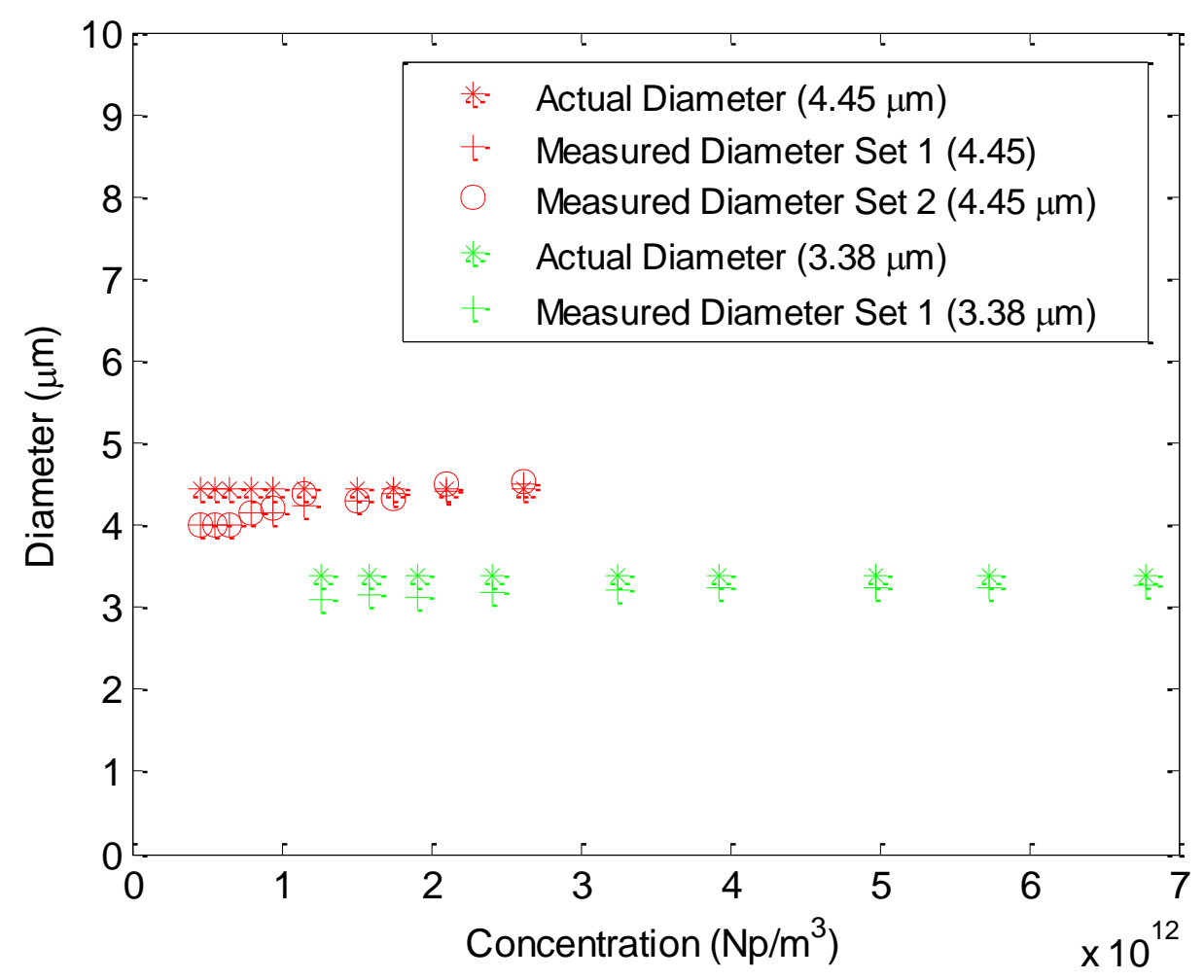

Figure 17: Particle diameter measured for mixed samples.

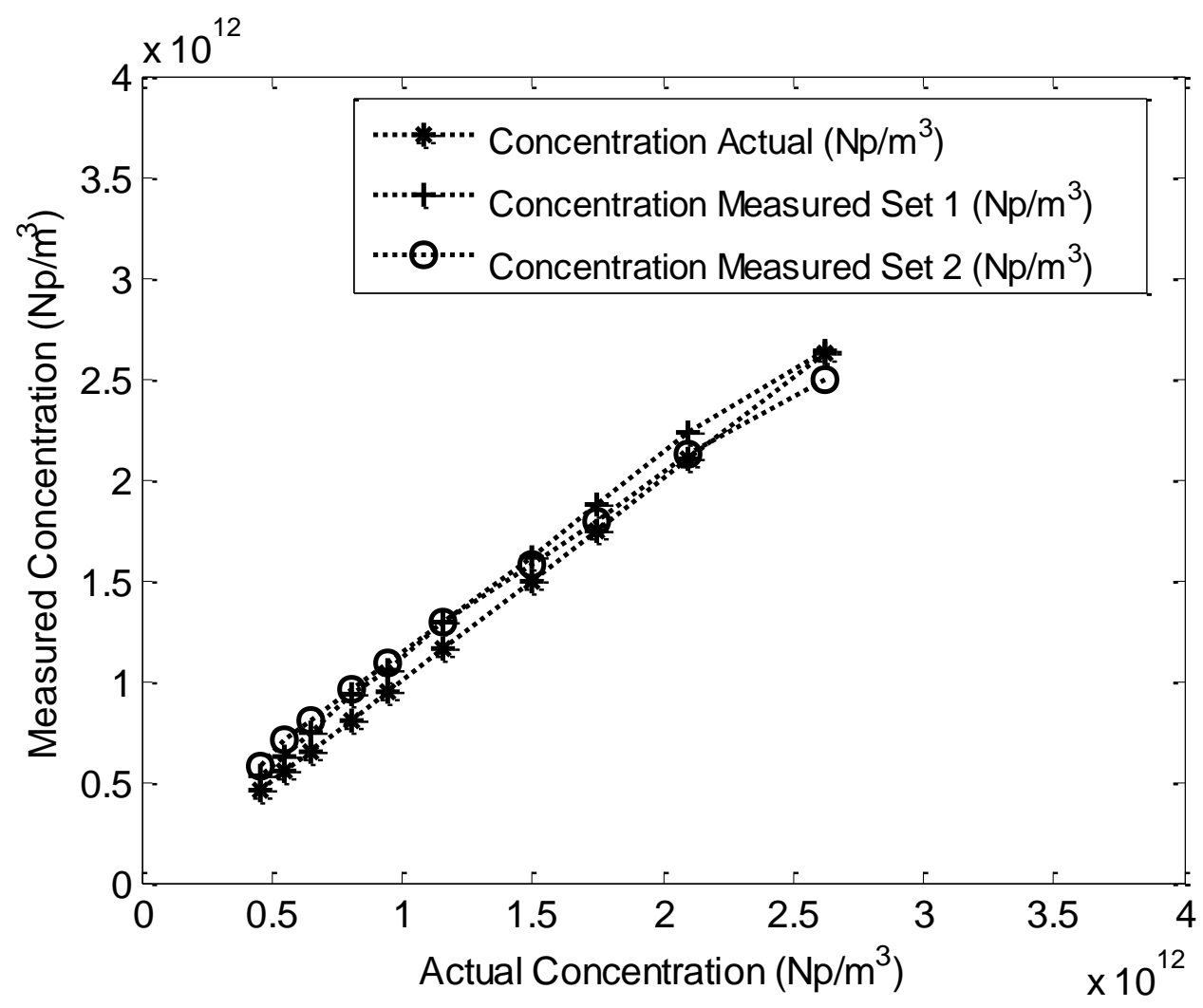

Figure 18: Particle concentration measured for mixed sample with mean diameter of $4.45 \mu \mathrm{m}$. 


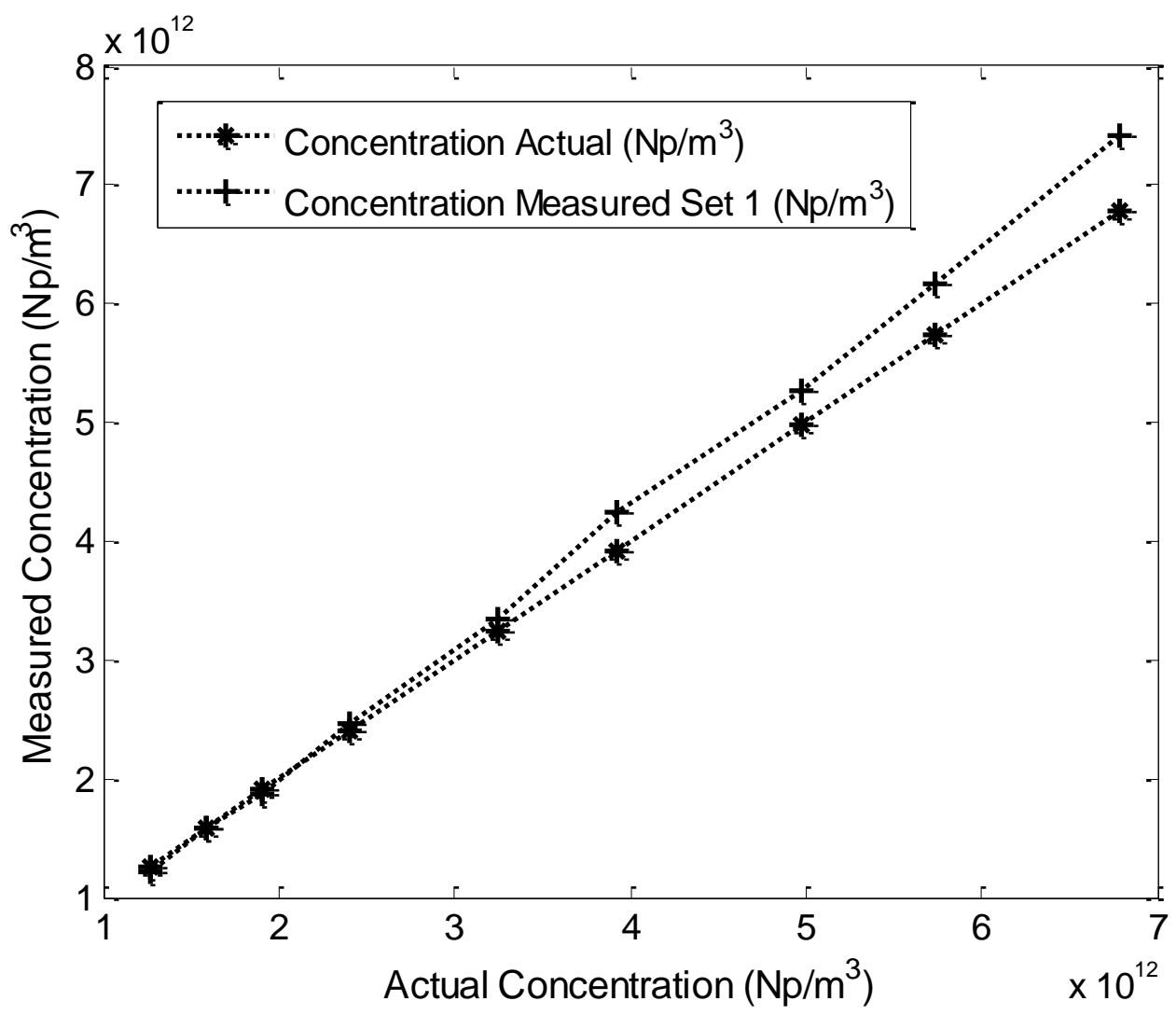

Figure 19: Particle concentration measured for the mixed sample with mean diameter of $3.38 \mu \mathrm{m}$. 


\section{PROOF OF CONCEPT EXPERIMENT 2: FLOW SETUP}

\section{Motivation for a flow experiment}

The second benchtop experiment was designed to characterize Polystyrene particle diameters and concentrations that are in motion. The aim of the experiment was to investigate whether the motion of particles would induce any error in diameter and concentration measurements. .

\section{Experimental Setup and Instrumentation}

In order to size test particles in flows, three particles sizes were purchased for analysis. The test particle sampled in the second experiment were mono-dispersed latex spheres of sizes 3.9 $\mu \mathrm{m}, 5.1 \mu \mathrm{m}$ and $7 \mu \mathrm{m}$ purchased from Magsphere Inc. The calibrated particles would provide a check on the measured diameters. These particles were available as $10 \%$ solids and were dispersed in an aqueous solution at high concentrations. The particle solution was then diluted using distilled water with an approximate ratio of 2:1. The dispersion medium for the particles was compressed air that was inducted into the experimental setup using a 3/8" flexible hose connected to the supply mains using a quick disconnect pipe fitting. The flow rate of compressed air was controlled by a needle valve installed in a $3 / 8$ " pipe and was measured using a pitot probe connected to an Omega Differential Pressure Transducer (PX142-001D5V) which had a maximum differential pressure detection range of $0-1$ psi. The regulated compressed air was then sent to a plenum chamber which was a cylindrical unit of height 19"and diameter 12". The Polystyrene particles which were initially suspended as an aqueous colloid were nebulized inside the plenum using an ultrasonic humidifier that created a dispersion of these particles inside the plenum chamber. A corrugated flexible tube was then used to transport the test particles from the plenum to a 1" pipe which was used to straighten the flow. The particles then exited the piping through a $3 / 4$ " reducer which created a $3 / 4$ " jet that contained particles. Particles in the jet were characterized using the optical components similar 
to those used in the static setup. The schematic of the test is shown below in Fig. (20), along with the actual final experimental setup in Fig (21).

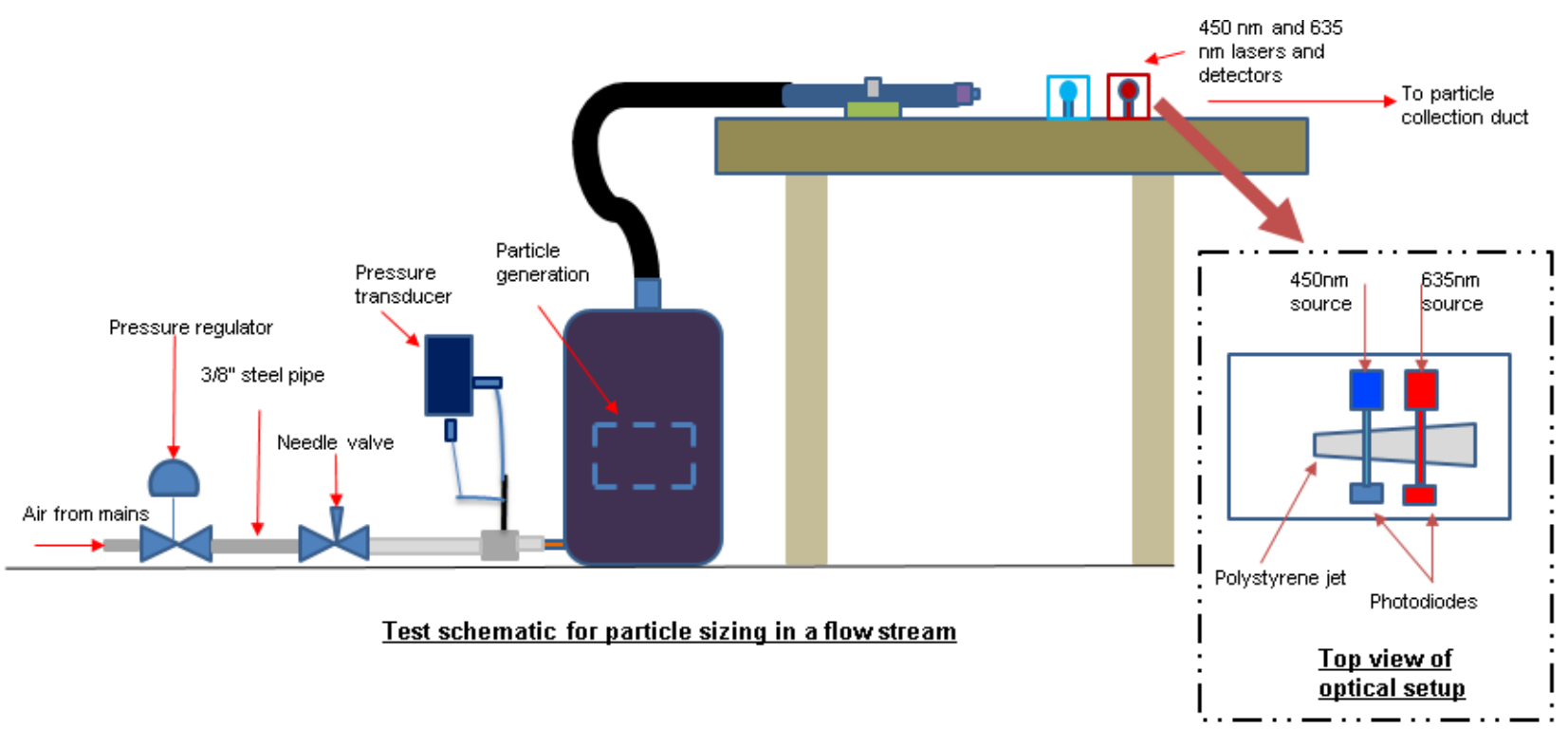

Figure 20: Test schematic of the second bench-top experiment used to size particles in flows.

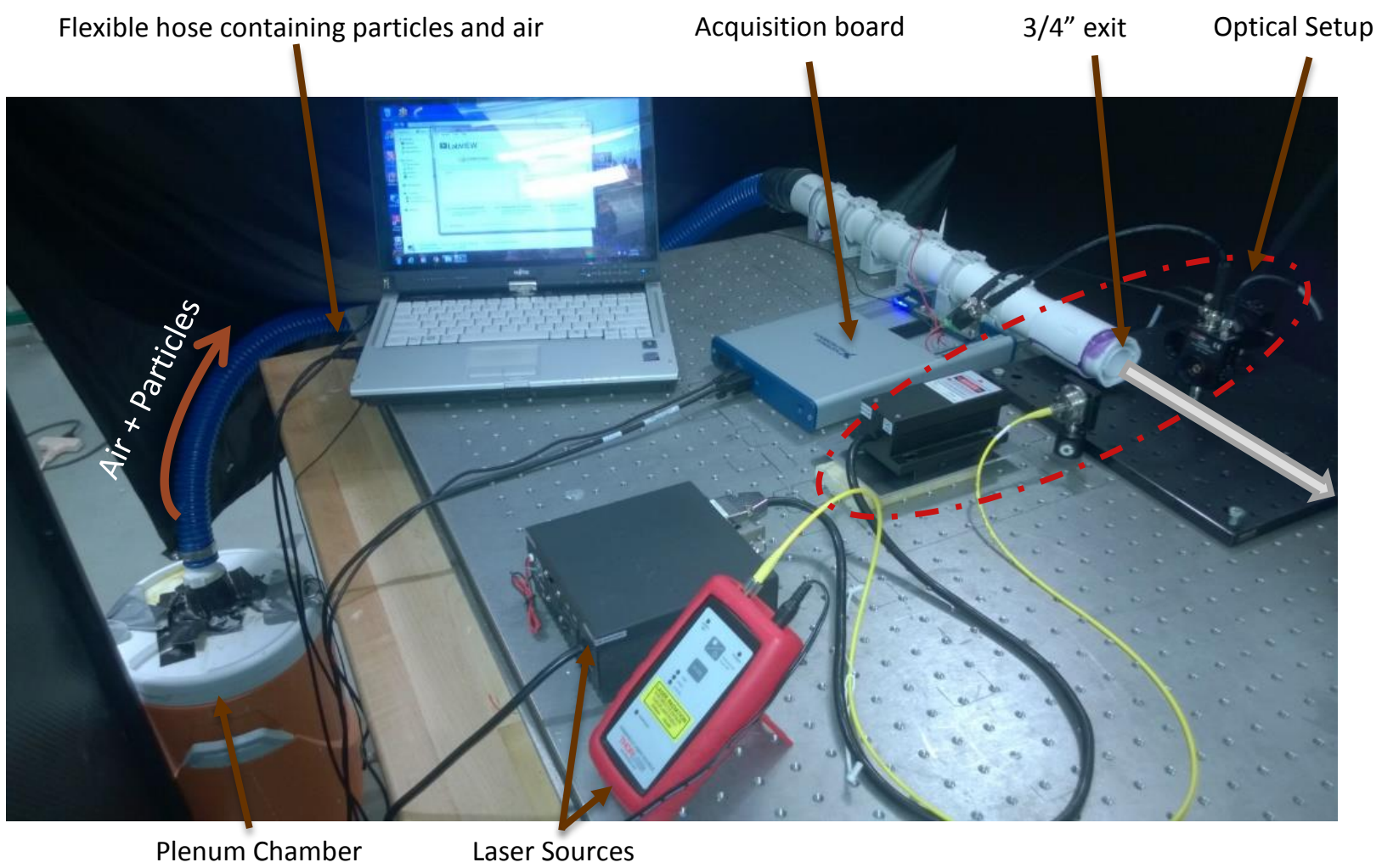

Figure 21: Actual picture of second bench-top experiment used to size particles in flows. 
The optical setup employed consisted of two continuous wavelength laser sources, a $447 \pm 5 \mathrm{~nm}$ (Dragon laser) with a power output of 500 milliwatts and a $635 \mathrm{~nm} \pm 10 \mathrm{~nm}$ (Thorlabs) handheld fiber- coupled laser with a power output of 2.5 milliwatts. The $447 \mathrm{~nm}$ laser had a TE00 transverse mode with a beam divergence of less than 1.5 milliradian and a beam diameter of 5 $\mathrm{mm}$. The sensor used to measure intensity of $447 \mathrm{~nm}$ light was a photodectector (Thorlabs PDA-25K ) that had an operating wavelength range of $150-550 \mathrm{~nm}$ and a rise time of 46 nanoseconds along with a noise equivalent power in the range of $7 \times 10^{-12}-3 \times 10^{-10}\left(\mathrm{~W} / \mathrm{H}_{\mathrm{z}}{ }^{1 / 2}\right)$. The maximum responsivity of this detector was $0.118(\mathrm{~A} / \mathrm{W})$ at $430 \mathrm{~nm}$ and the active area of the detector was $4.8 \mathrm{~mm}^{2}$. In order to focus the $447 \mathrm{~nm}$ laser light onto the detector, a combination of plano convex and plano concave lenses with focal lengths of $f=+100 \mathrm{~mm}$ and $f=-50 \mathrm{~mm}$ respectively were used. These two lenses were separated by a distance of $50 \mathrm{~mm}$. The 635nm fiber coupled laser used an aspheric fiber port (Thorlabs PAF-X-7-A) for collimation of the laser beam. The collimated laser beam had a divergence of 0.467 milliradians. The $635 \mathrm{~nm}$ light was collimated using an aspheric fiber port (Thorlabs PAF-X7-A) and its intensity was measured using a Thorlabs PDA-36A light detector after it traversed the jet containing particles. This photodiode had a responsivity of $0.4137 \mathrm{~A} / \mathrm{W}$ at a wavelength of $635 \mathrm{~nm}$ and an active area of $13 \mathrm{~mm}^{2}$. Output from the two detectors was then recorded using a National Instruments NI USB 6351 acquisition board which had a 16 bit resolution and a maximum signal input voltage of $10 \mathrm{~V}$. Extinction due to the water present in the beam was characterized by measuring the light beam intensity without any particles in the jet. The optical setup is shown in Fig. (22), below. 


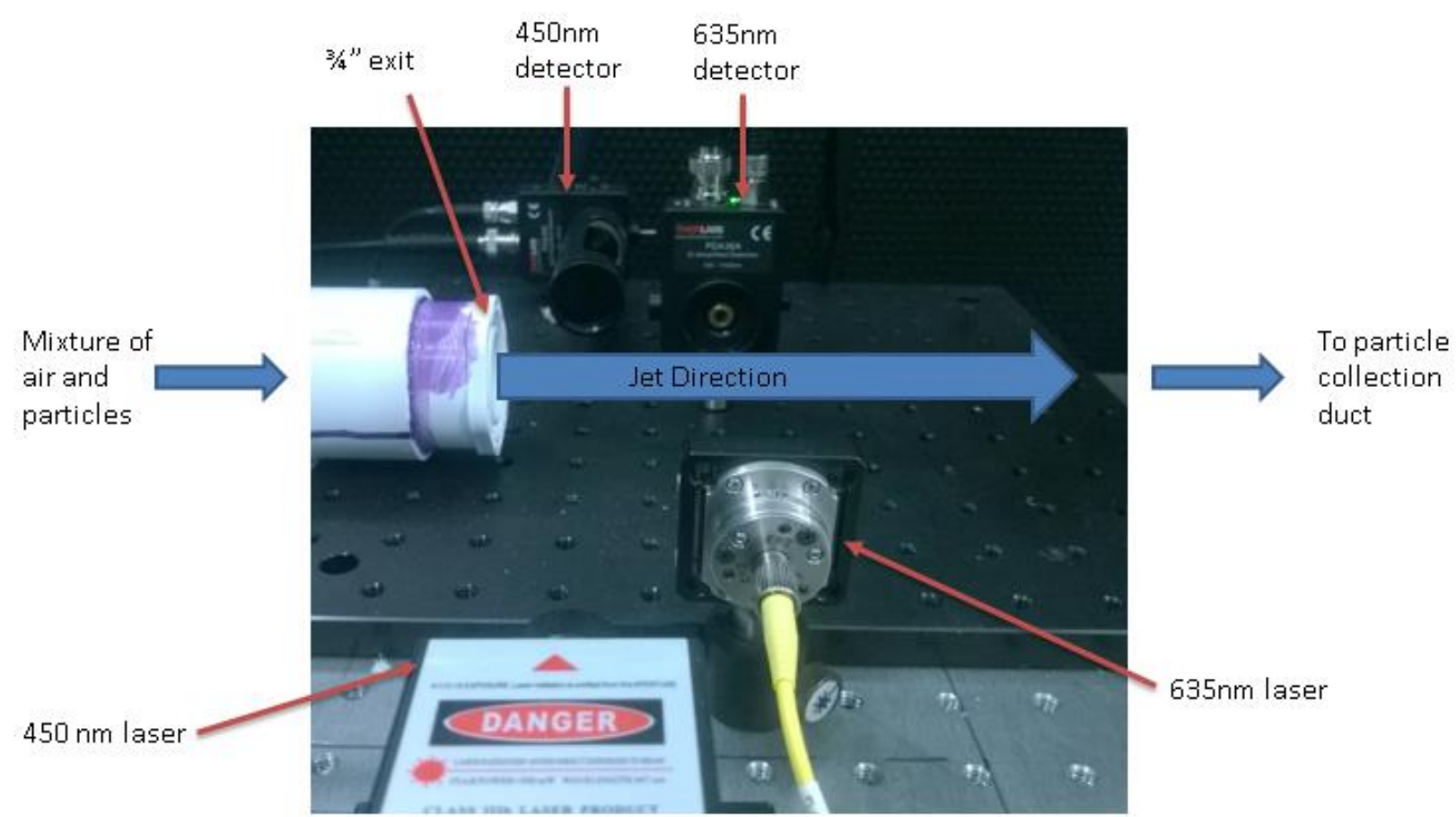

Figure 22: Optical setup used in the second experiment used to size particles in flows.

The output voltage from the photodiodes was converted into power using a LabVIEW virtual interface that converts the measured voltage into power while acquiring data. The values of power obtained using the two photodiodes was validated using an optical power meter. In order to obtain transmissivities as given by Eq. (1), power of the laser beam was measured with and without the presence of particles in the beam path. The ratio of the incident light intensity without the particles $\left(I_{o}\right)$ to the transmitted light intensity in the presence of the sample $\left(I_{t}\right)$ was then recorded assuming the beam waist was constant for the two laser light sources. This allows the following assumption for a given wavelength source:

$$
\frac{I_{t}}{I o}=\frac{P_{t}}{P_{o}}=\tau_{i}
$$

\section{Equation 9}

Where $P_{t}$ and $P_{o}$ are the transmitted and incident power on the photodiodes and $\tau_{\mathrm{i}}$ is the transmissivity at wavelength $\lambda_{\mathrm{i}}$. The resultant ratio of transmissivities $(\mathrm{R})$ was obtained by 
taking the ratio of the two individual transmissivities $(\tau)$ obtained experimentally at the two wavelengths of light (447 nm and $635 \mathrm{~nm}$ ). The mean particle diameter was obtained by inferring particle diameters from the ratio $(\mathrm{R})$ using the functional relationship between diameters and average extinction efficiency as expressed by Eq. (3) and graphically represented in Fig. (5).

\section{Results from the Flow Setup (Mono-dispersed Particle Samples)}

As described previously, the test particles were pre-calibrated using a Scanning Electron Microscope. This would provide a check against the diameters measured using the extinction technique. Intensity of light was measured with and without the presence of particles in the jet and a ratio of the two quantities was taken. This ratio was then used to determine the average particle diameters by using the relationship between the ratio of transmissivities and diameters as described in the measurement concept section. It was observed that the ultrasonic humidifiers used for nebulizing the particles were not effective in dispersing polystyrene particles when the velocity of jet increased to a value greater than $5 \mathrm{~m} / \mathrm{s}$. Hence in order to obtain uniform quantities of seed particles in the measurement volume, measurements were performed at a velocity of less than $5 \mathrm{~m} / \mathrm{s}$. Results from particle sizing measurements performed using the flow setup are represented below. It is important to note that the extinction measurement technique is independent of velocity and relies on an average intensity signal measured by the photodiodes and hence the results can be scaled up to higher velocities of particles as seen by an aircraft engine inlet. 


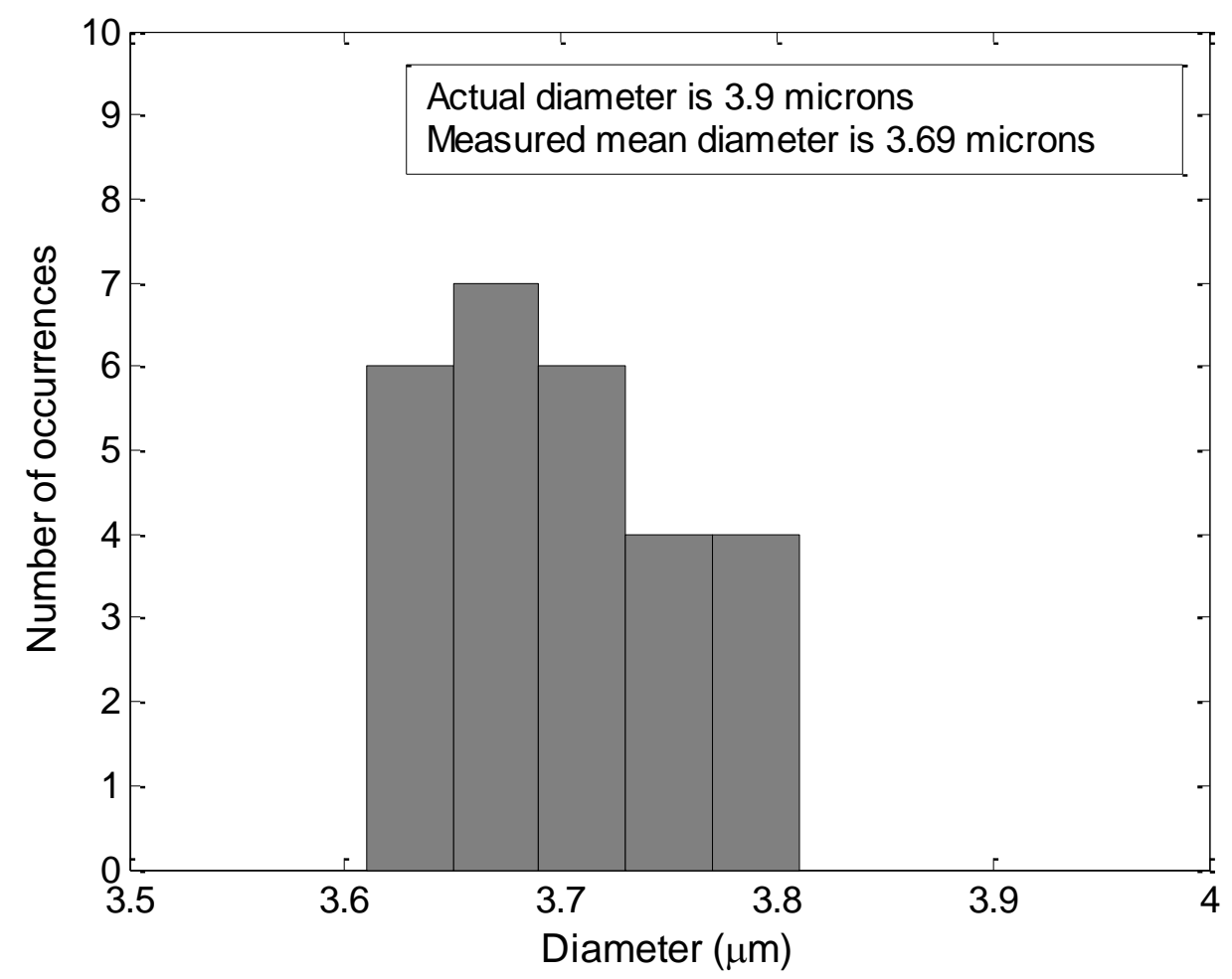

Figure 23: Histogram of $3.9 \mu \mathrm{m}$ particles measured at velocities under $5 \mathrm{~m} / \mathrm{s}$.

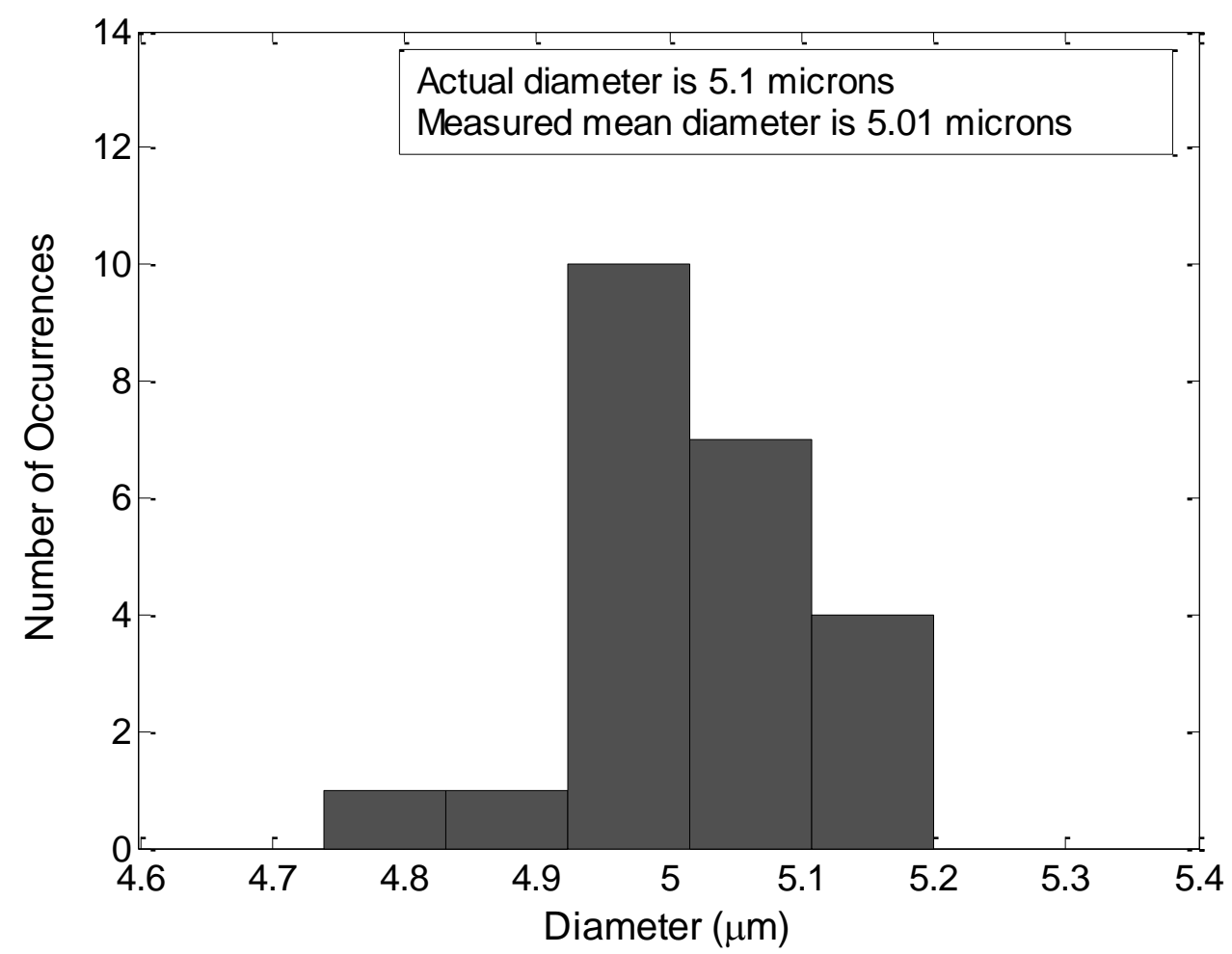

Figure 24: Histogram of $5.1 \mu \mathrm{m}$ particles measured at velocities under $5 \mathrm{~m} / \mathrm{s}$. 


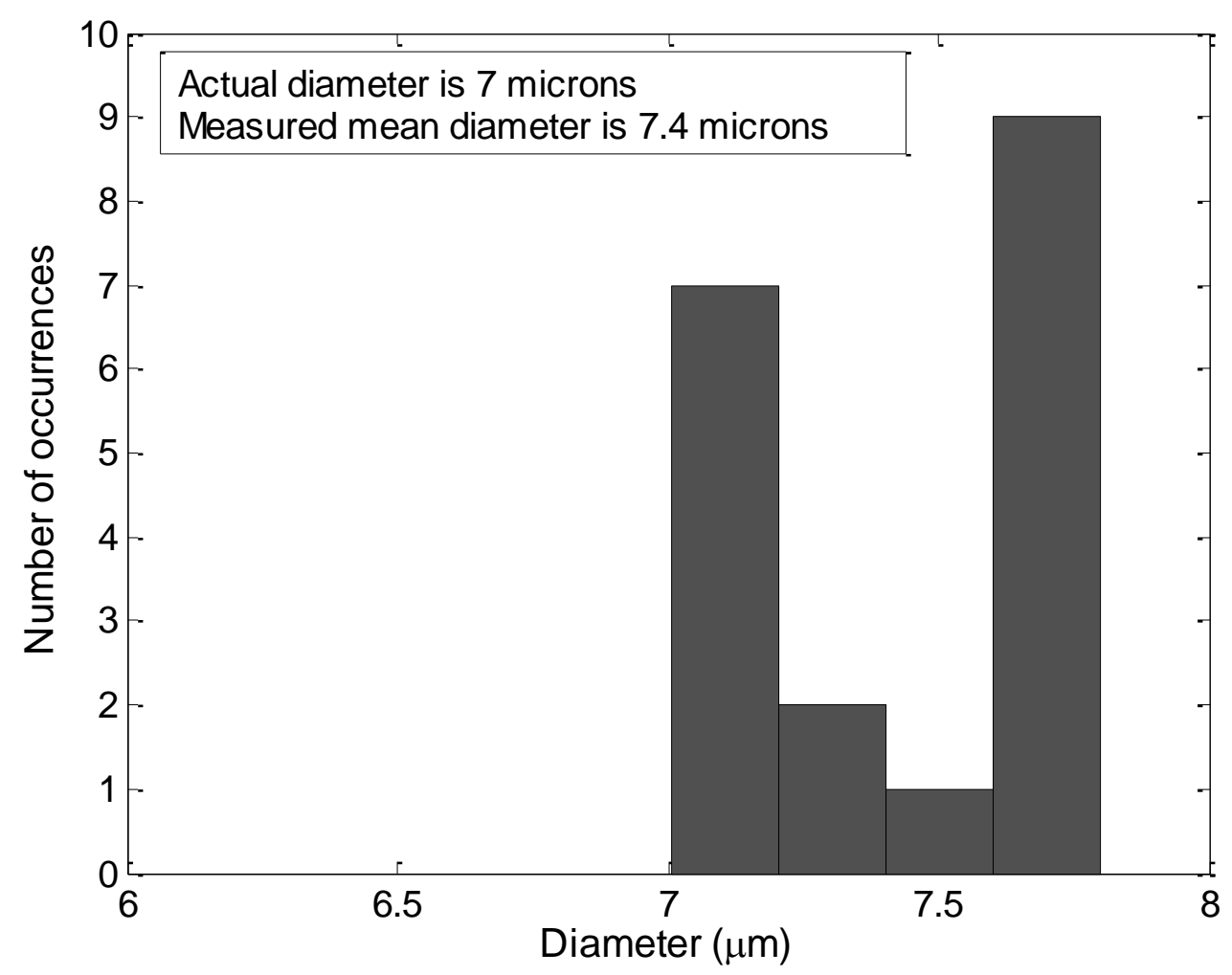

Figure 25: Histogram of $7 \mu \mathrm{m}$ particles measured at velocities under $5 \mathrm{~m} / \mathrm{s}$.

As seen from the results presented above, diameters are predicted with a high accuracy for all particles sampled. The maximum relative error in the diameters sampled is $6 \%, 7 \%$ and $11 \%$ for $3.9 \mu \mathrm{m}, 5.1 \mu \mathrm{m}$ and $7 \mu \mathrm{m}$ particles respectively. One of the reasons for deviation in diameter measurements could be attributed to the low spectral gap between the two laser sources of light used. This narrow gap results in an ambiguity in measurements as shown in Fig. (A1) and a small variation in intensity loss measured by the photodiodes results in variations in diameters measured. The over prediction in diameter measured as seen the case of $7 \mu \mathrm{m}$ particles could be due to the coagulation of particles as they flow through the sample volume thereby resulting in an error. The constant under prediction of the $3.9 \mu \mathrm{m}$ particle sample data could be attributed to the lack of uniform particle seeding in the measurement volume thereby resulting in a higher intensity light incident on the photodiodes that results in the technique reporting a lower diameter than the actual particle diameter. 


\section{Measurement of Diameters and Concentrations in a Poly-dispersed Sample}

This far in the flow experiment, results reported were for mono-dispersed samples. It is important that the technique be able to resolve a mean diameter from a poly-dispersed sample of polystyrene in varying concentrations. Three poly-dispersed particle samples of sizes between $(3.9 \mu \mathrm{m}$ and $7 \mu \mathrm{m})$ were prepared using the relation below for a weighted Sauter mean diameter:

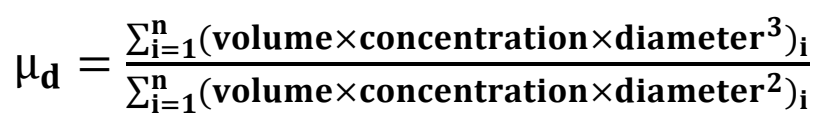

\section{Equation 10}

Where $\mathrm{n}$ is the number of particle samples of different sizes used to create the poly-dispersed sample and $\mu_{d}$ is the mean diameter of the polydispersed sample prepared. This statistical mean diameter would provide a validation check on the results obtained from the extinction measurements. The three batches prepared had a mean diameter of $4.41 \mu \mathrm{m}, 5 \mu \mathrm{m}$ and $5.9 \mu \mathrm{m}$. Utilizing the experimental procedure described above to size mono-dispersed samples, mean diameters were obtained for the poly-dispersed samples prepared and are shown in Figs. (2628). As seen from the figures, diameters and concentrations of the mixture were predicted in close conformance with the actual values. The deviation in measurements can be attributed to the spectral gap between the two lasers resulting in an error in measurement due to the measured diameter being sensitive to the intensity loss of the laser light. There may also be an error in diameter prediction due to the coagulation of particles as they flow through the measurement volume. 


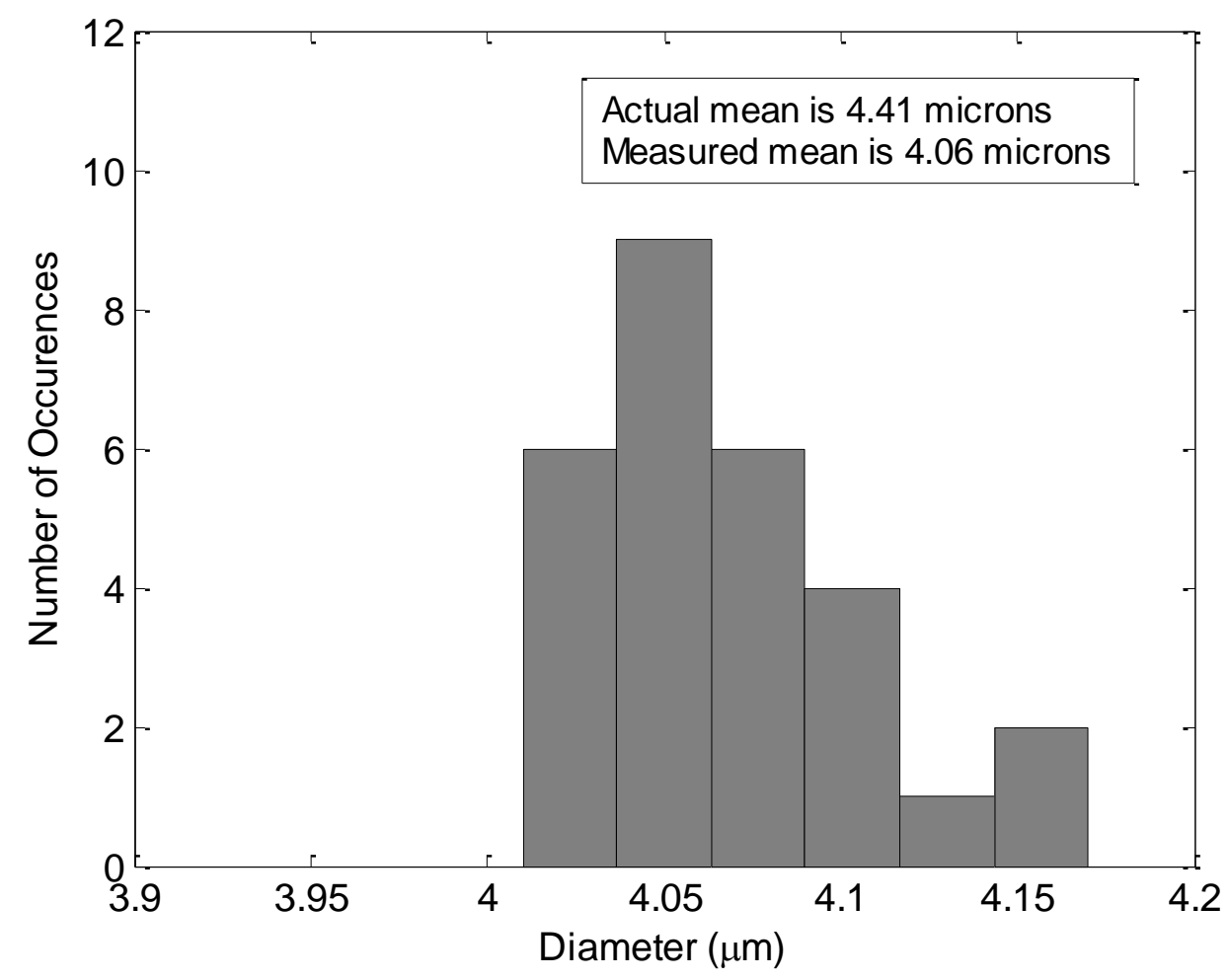

Figure 26: Histogram of poly disperse particle samples with mean diameter of $4.41 \boldsymbol{\mu m}$.

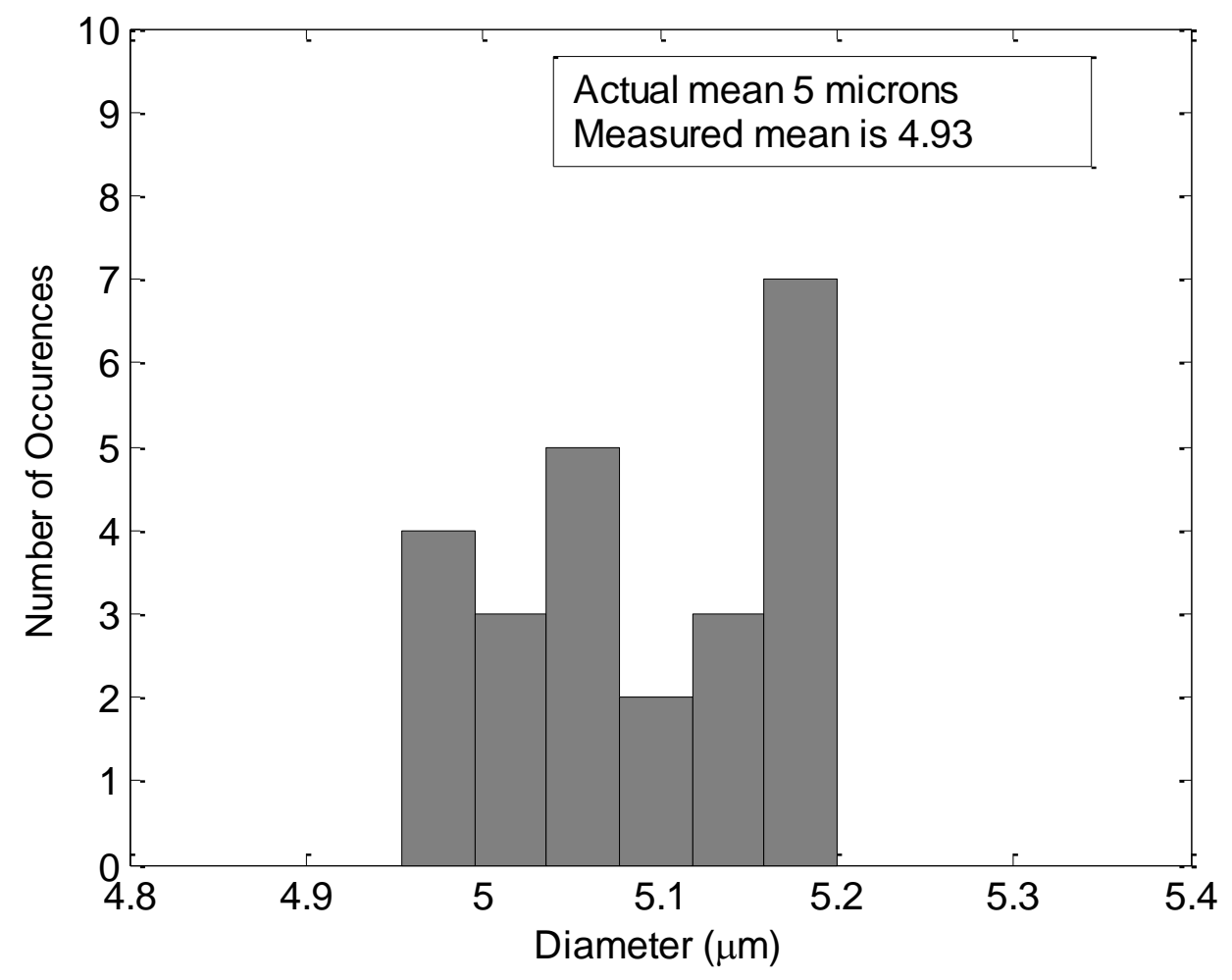

Figure 27: Histogram of poly disperse particle samples with mean diameter of $5 \mu \mathrm{m}$. 


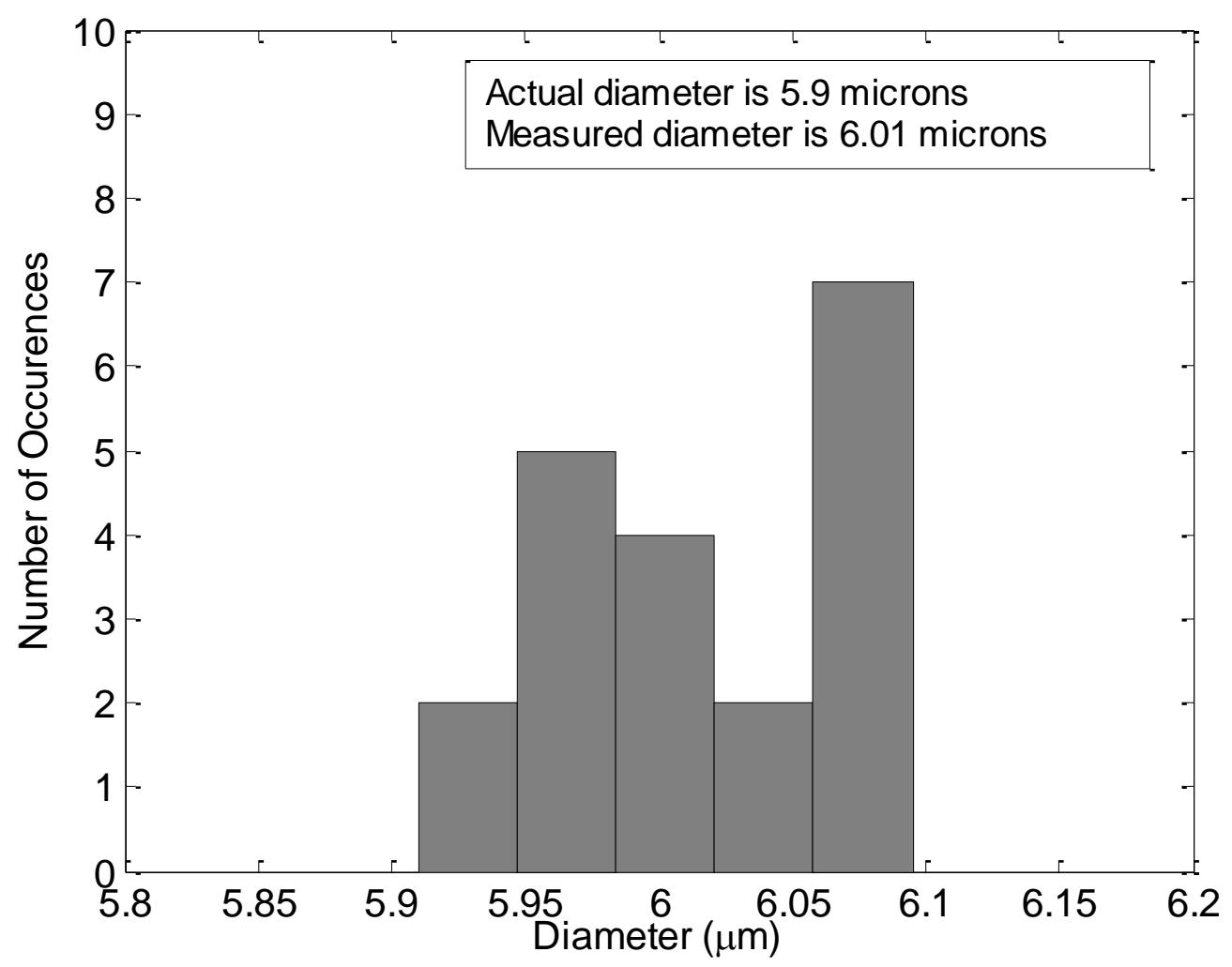

Figure 28: Histogram of poly disperse particle samples with mean diameter of $6.01 \mu \mathrm{m}$. 


\section{EXPERIMENTAL UNCERTAINTY}

\section{Dependence of Diameters and Concentrations on Parameters Utilized in Analysis}

For all diameter and concentration measurements, it becomes imperative that the technique used in characterization of particles be able to resolve the measured data with low uncertainties as large measurement uncertainties would greatly reduce the applicability of the technique to real world applications. This section will aim to quantify uncertainties in measurements performed for the flow experiment as well as the static experiment.

In order to identify the sources of uncertainty and variability in measurements, it is important to recall Eq. (1) and Eq. (3), the two equations used extensively in the sizing of particles as well as obtaining concentrations.

$$
\begin{array}{cc}
\tau_{i}=-\ln \left(\frac{I_{t}}{I_{o}}\right)=\frac{\pi}{4} C_{n} L \int_{0}^{\infty} Q\left(\pi D / \lambda_{i}, m\right) f(D) D^{2} d D, & \text { Equation } 1 \\
R_{i j}=\frac{\tau_{i}}{\tau_{j}}=\frac{\bar{Q}\left(\lambda_{i}, D_{32}\right)}{\bar{Q}\left(\lambda_{j}, D_{32}\right)} & \text { Equation 3 }
\end{array}
$$

From the above equations it can be observed that diameter measurements depend on the ratio of the attenuation loss at two wavelengths, which in turn depend on:

1. The ratio of intensity loss at the two wavelengths used ( $\mathbf{I}_{\mathbf{t}}$ and $\left.\mathbf{I}_{\mathbf{0}}\right)$.

2. The wavelength of light utilized $\left(\boldsymbol{\lambda}_{\boldsymbol{i}}\right.$ and $\left.\boldsymbol{\lambda}_{\boldsymbol{j}}\right)$.

3. The refractive index of the test particles (m).

Concentration measurements on the other hand depend on light attenuation at one wavelength utilized, which in turn depend on:

1. The ratio of intensity loss at the two wavelengths used ( $\mathbf{I}_{\mathbf{t}}$ and $\left.\mathbf{I}_{\mathbf{o}}\right)$. 
2. The wavelength of light utilized $\left(\lambda_{i}\right.$ and $\left.\lambda_{j}\right)$.

3. The refractive index of the test particles (m).

4. The path length of light travelled during the extinction measurement (L).

\section{Uncertainty in Diameter Measurements}

The effect of parameters affecting diameter measurements as described above were taken into account one by one while keeping all other parameters constant. This technique resulted in a perturbation of individual effects as described by R.J. Moffat [24]. Finally the effect of all parameters was obtained using an RMS value of each measurement uncertainty. This analysis is performed on experiments carried out using the $450 \mathrm{~nm}$ and $635 \mathrm{~nm}$ lasers as well as the two laser sources that may be used in the future, $450 \mathrm{~nm}$ and $7000 \mathrm{~nm}$.

Effect of wavelength: The wavelength of the two lasers employed did not fluctuate to a value that would be responsible for deviation in the measurement. Hence the effect was not considered in the uncertainty analysis.

Effect of power fluctuation in photodiodes: The power measured by the photodiodes fluctuated within a certain range during the measurements and this could lead to errors in the measurement of diameters. Among all the data sets available, the worst case scenario of power fluctuation was utilized and input into the analysis. This worst case scenario was decided by the ratio of the variation of power to the range of power in the measurements. The error arising due to photodiode power fluctuations resulted in a variation in the ratio of laser light attenuation at the two wavelengths sources (R) used and consequently resulted in a diameter error. The maximum variability in the ratio $(\mathrm{R})$ due to the power fluctuations was taken into account and applied to all the data sets.

Effect of refractive index: The effect of an unknown refractive index or a variation of the refractive index with temperature may result in a deviation in diameter measurements since the algorithm to construct data tables for the ratio of extinction efficiencies (R) requires the 
refractive index as an input. The refractive index may be represented as an imaginary number $m=a+i b$ where $a$ is the real component of the refractive index and $b$ is the imaginary component of the refractive index that is responsible for light absorption. It was observed that variation of the imaginary component in the refractive index did not result in a significant deviation in diameter measurements and hence was not considered [12]. For the uncertainty analysis, refractive index of the known Polystyrene particles was varied by $\pm 5 \%$ and the effect on the final diameter was considered in the uncertainty analysis. It was noted that among all factors that contribute to errors in the measurement, the effect of an unknown refractive index was most profound and resulted in relative errors in diameter of up to $17 \%$.

Final results of the uncertainty analysis indicate low relative errors in diameter obtained due to sources of variability described earlier. These errors are represented in Figs. (29 -30), below for the wavelength sources used as well as the two wavelength sources to be used in the future.

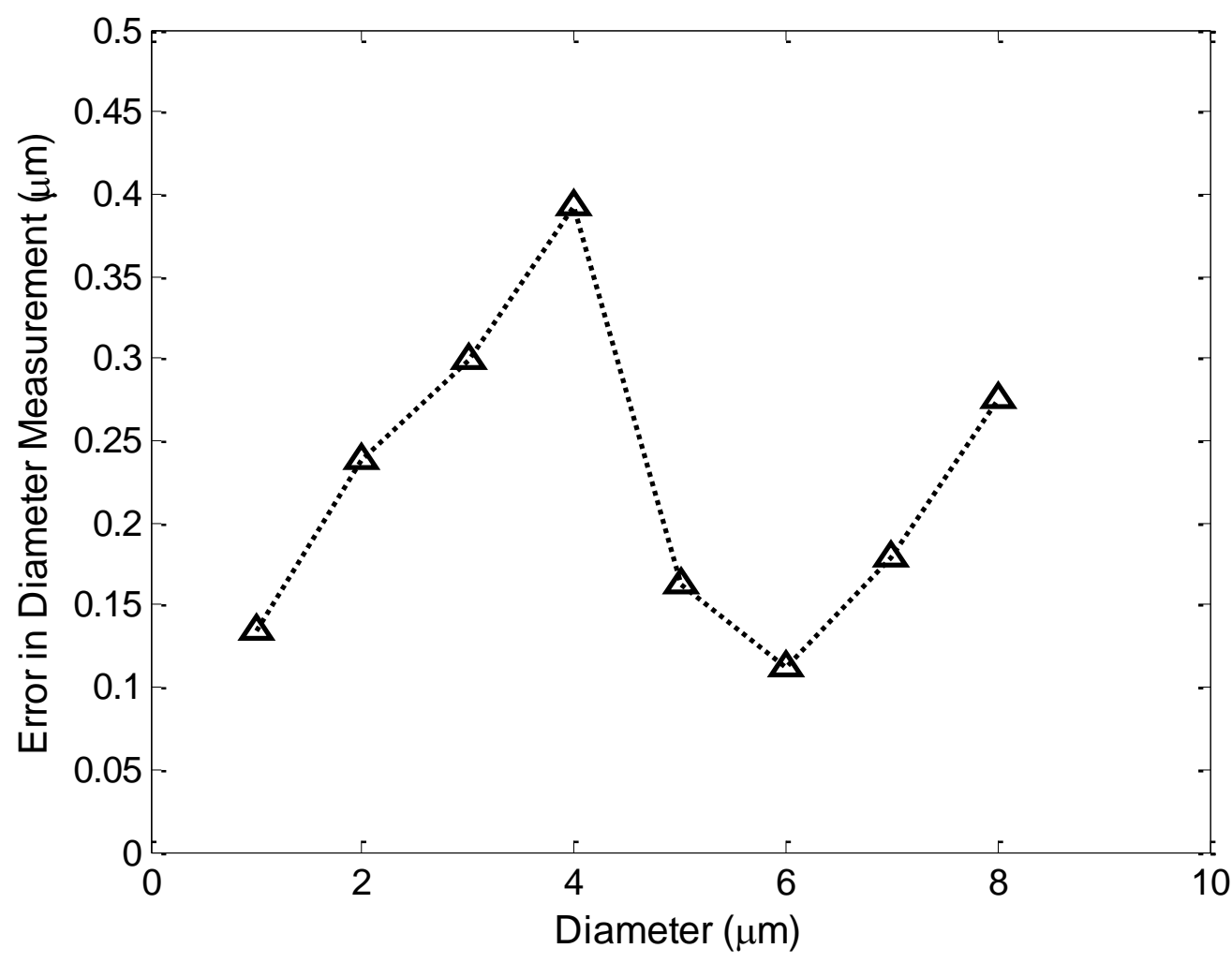

Figure 29: Error in diameter measurement due to sources of uncertainty using the current setup of $447 \mathrm{~nm}$ and $635 \mathrm{~nm}$ lasers. 


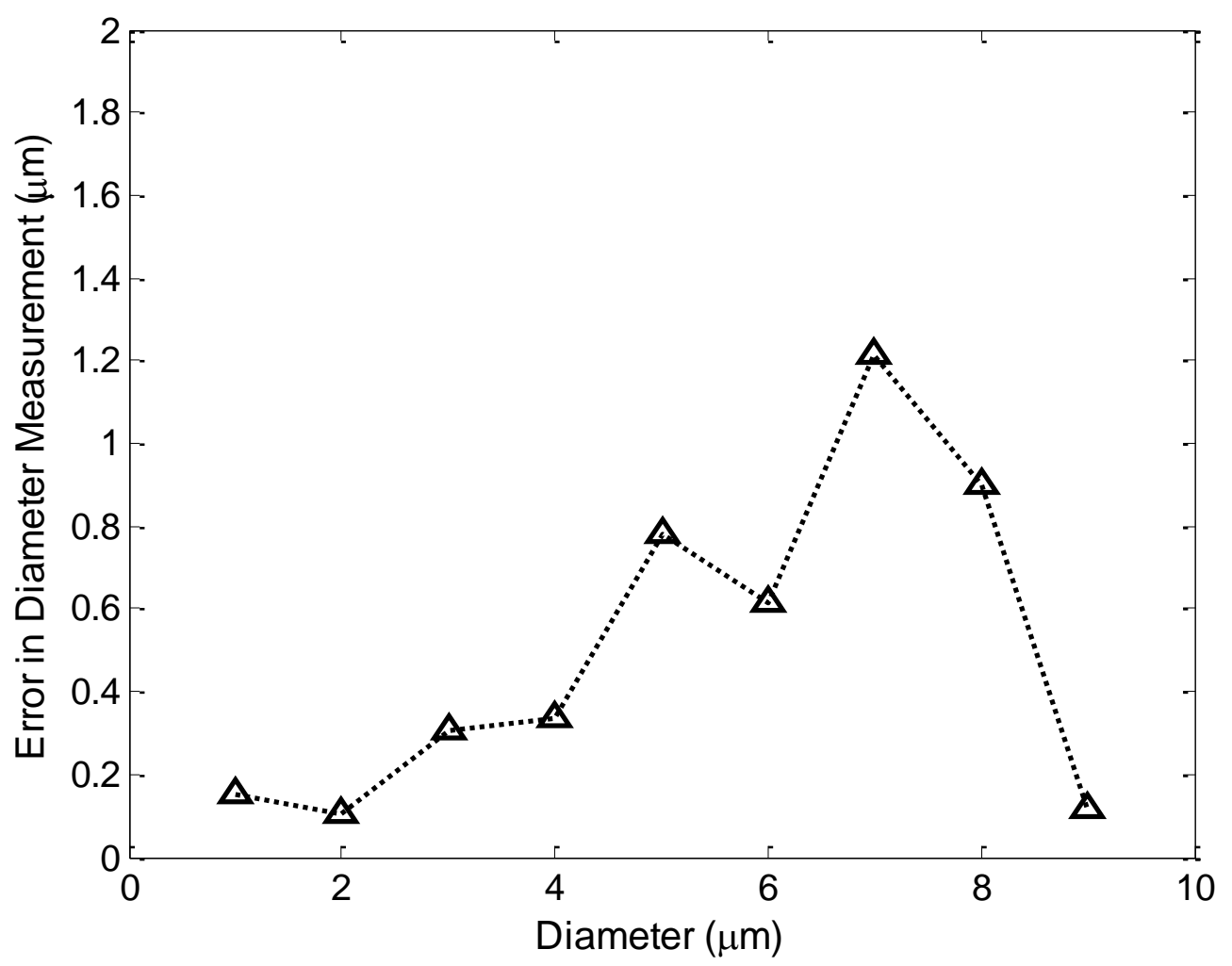

Figure 30: Error in diameter measurement due to sources of uncertainty using $447 \mathbf{~ n m}$ and $7000 \mathrm{~nm}$ lasers to be used in the future.

On closely observing the errors in measurements due to the sources of variability for the current and future setup, it can be noted that the maximum deviation in diameter measurement results for the diameter of $7 \mu \mathrm{m}$ particle size. This deviation can be attributed to the behavior of the ratio of attenuation vs diameter curve when the refractive index is varied. The variation in refractive index resulted in a deviation of the ratio of transmissivities and this effect was pronounced in the case of the $447 \mathrm{~nm}$ and $7000 \mathrm{~nm}$ laser light where the effect of refractive index variation was most pronounced at diameter values between 5 and $7 \mu \mathrm{m}$. The uncertainty analysis performed on the data obtained shows that the technique to obtain diameters may be sensitive to an unknown refractive index and the effect of its variation can be detrimental to obtaining highly accurate data. The maximum relative error in diameter measurements on account of all possible sources of variability was $17 \%$ obtained for a $7 \mu \mathrm{m}$ particle diameter. 


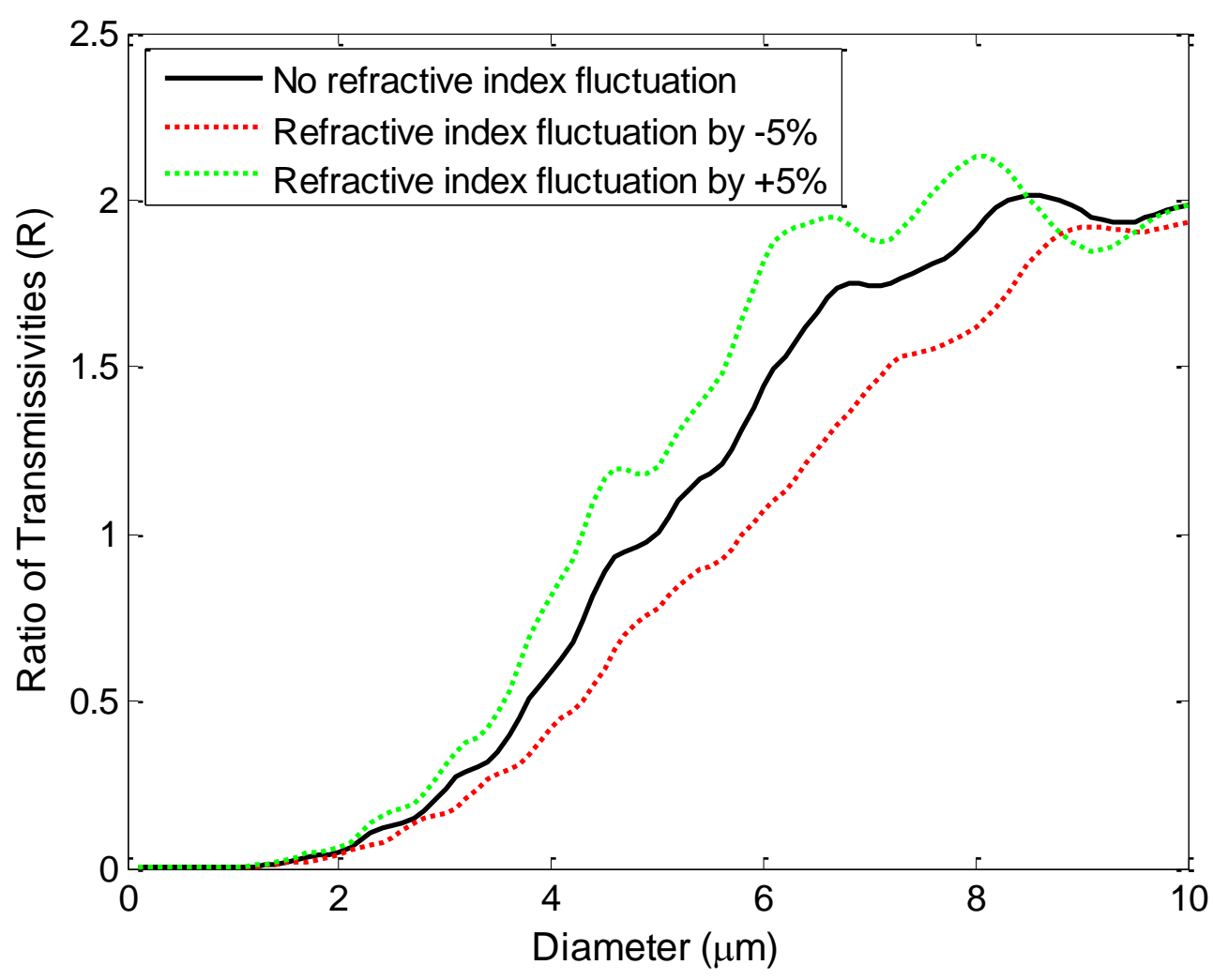

Figure 31: Plot showing the ratio of transmissivity (R) vs. diameters demonstrating the effect of refractive index variation on diameter measurements shown for $447 \mathrm{~nm}$ and $7000 \mathrm{~nm}$ lasers.

\section{Uncertainty in Concentration Measurements}

The effect of parameters affecting concentration measurements as described above were taken into account one by one while keeping all other parameters constant. As descried in the section for diameter uncertainty, this technique resulted in a perturbation of individual effects as described by R.J. Moffat [24]. Finally the effect of all parameters was obtained using an RMS value of each measurement uncertainty. Again, this analysis was performed on experiments carried out using the $450 \mathrm{~nm}$ and $635 \mathrm{~nm}$ lasers as well as the two laser sources to be used in the future, $450 \mathrm{~nm}$ and $7000 \mathrm{~nm}$. It should be noted that since the concentration predictions depend on the diameter measurements, an error in diameter measurements would affect the concentrations measured and lead to errors in its prediction. As opposed to the diameter uncertainties, the concentration uncertainties were recorded only for the particle diameter sized. 
Effect of wavelength: The wavelength of the two lasers employed did not fluctuate to a value that would be responsible for deviation in the measurement. Hence the effect was not considered in the uncertainty analysis.

Effect of power fluctuation in photodiodes: The power measured by the photodiodes fluctuated within a certain range during the measurements and this led to errors in the measurement of diameters and consequently concentrations. As described above, among all the data sets available, the worst case scenario of power fluctuation was utilized and input into the analysis. This worst case scenario was decided by the ratio of photodiode power variation to the range of power in measurements obtained. The maximum variability in the ratio $(\mathrm{R})$ due to the power fluctuations was taken into account and applied to all the data sets.

Effect of refractive index: The effect of an unknown refractive index or a variation of the refractive index with temperature may result in a deviation in diameter and consequently concentration measurements since the algorithm to construct data tables for the ratio of extinction efficiencies $(\mathrm{R})$ requires the refractive index as an input. The refractive index may be represented as an imaginary number $m=a+i b$ where $a$ is the real component of the refractive index and $b$ is the imaginary component of the refractive index that is responsible for light absorption. As described in the uncertainty measurements for diameter, the effect of imaginary components was not considered. For the uncertainty analysis, refractive index of the known Polystyrene particles was varied by $\pm 5 \%$ and the effect on the final concentrations was considered in the uncertainty analysis.

Effect of path length: Concentration measurements are also dependent on the distance the light has to travel between the laser source and the corresponding detector, described in Eq. (1). An inaccurate measurement of the extinction path length, results in a mis-prediction of concentration values and hence was considered in the uncertainty analysis. 
As seen from the figure below, concentration measured shows a decreasing trend for an increasing diameter across all the three laser sources used. An interesting observation is that the uncertainty in concentration measurements progressively increases with an increasing wavelength of light source used with the $7000 \mathrm{~nm}$ laser being the least reliable of the three laser sources used. This increased error in the concentration measured is due to the variation of the kernel function, $\mathrm{Q}_{\text {ext }}$ in Eq. (1). The variation of $\mathrm{Q}_{\mathrm{ext}}$ (the kernel) as a response to the variation of parameters contributing to uncertainty as described above goes on increasing with an increase in the wavelength. This leads to increased variability in concentrations measured and consequently results in increased errors.

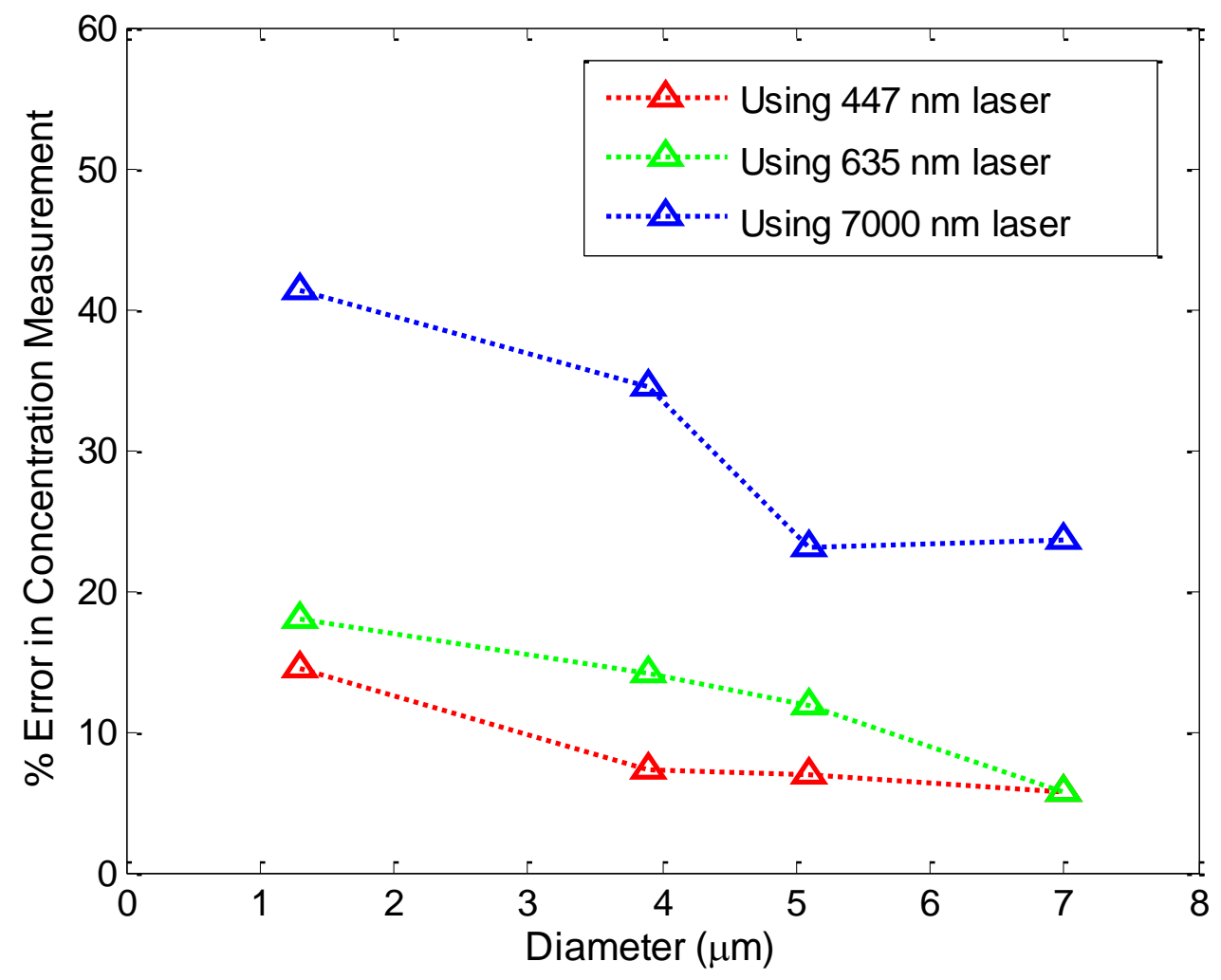

Figure 32: Percentage errors in concentration measurements due to various sources of uncertainty using $447 \mathrm{~nm}, 635 \mathrm{~nm}$ and $7000 \mathrm{~nm}$ lasers. 
Thus from the concentration uncertainty analysis, it can be concluded that the shorter wavelength lasers should be used for more reliable concentration measurements. It should also be noted that the uncertainty analysis performed here was for the Polystyrene test particles. The same procedure must be repeated while analyzing other test particulate matter. 


\section{FUTURE WORK PROPOSED}

\section{Simultaneous Particle Diameter and Concentration Measurements in Flows}

Thus far in flow experiments, all measurements were performed to show the capability of the technique to resolve diameters in close conformance to actual values as specified by the manufacturer. Concentrations from the flow experiments were calculated from Eq. (1) by using information of the diameters measured. However, they were not reported as a method to verify the concentration of particles did not exist for the flow experiments. In order to report the concentration measured, a particle counting system was setup. This setup will utilize the light from the $447 \mathrm{~nm}$ laser being used for extinction measurements. A light chopper (Thorlabs MC 1000 Optical Chopper) will be used to chop the continuous wavelength laser and create a pulsed source. For the average camera, a long exposure time to account for intense illumination intensity may lead to blurred images while operating which will occur if the laser is operated in Continuous Wavelength $(\mathrm{CW})$ mode as the response of the sensor on board the camera may not be quick enough to account for the intense background illumination. The pulses created from the chopper will aid in preventing blurred images obtained from the camera if used with the laser in Continuous Wavelength $(\mathrm{CW})$ mode by providing intermittent illumination and will also freeze the particles in motion thereby reducing the possibility of motion blur. The camera placed over the laser beam will capture images of the illuminated test particles as they passed through the measurement volume. These images will then be processed using software to count the particles in the beam. The number density of particles obtained using the extinction technique will be verified with the concentration of particles obtained from the camera measurements. Test particles used for these measurements are $3.9 \mu \mathrm{m}$ polystyrene latex spheres dispersed in a distilled water medium. As described in the previous section, these particles will be nebulized using an ultrasonic humidifier and sampled using the extinction technique in a jet 
containing the sampled particles and air moving at a velocity of under $5 \mathrm{~m} / \mathrm{s}$. The schematic of this setup is shown below followed by the actual setup.

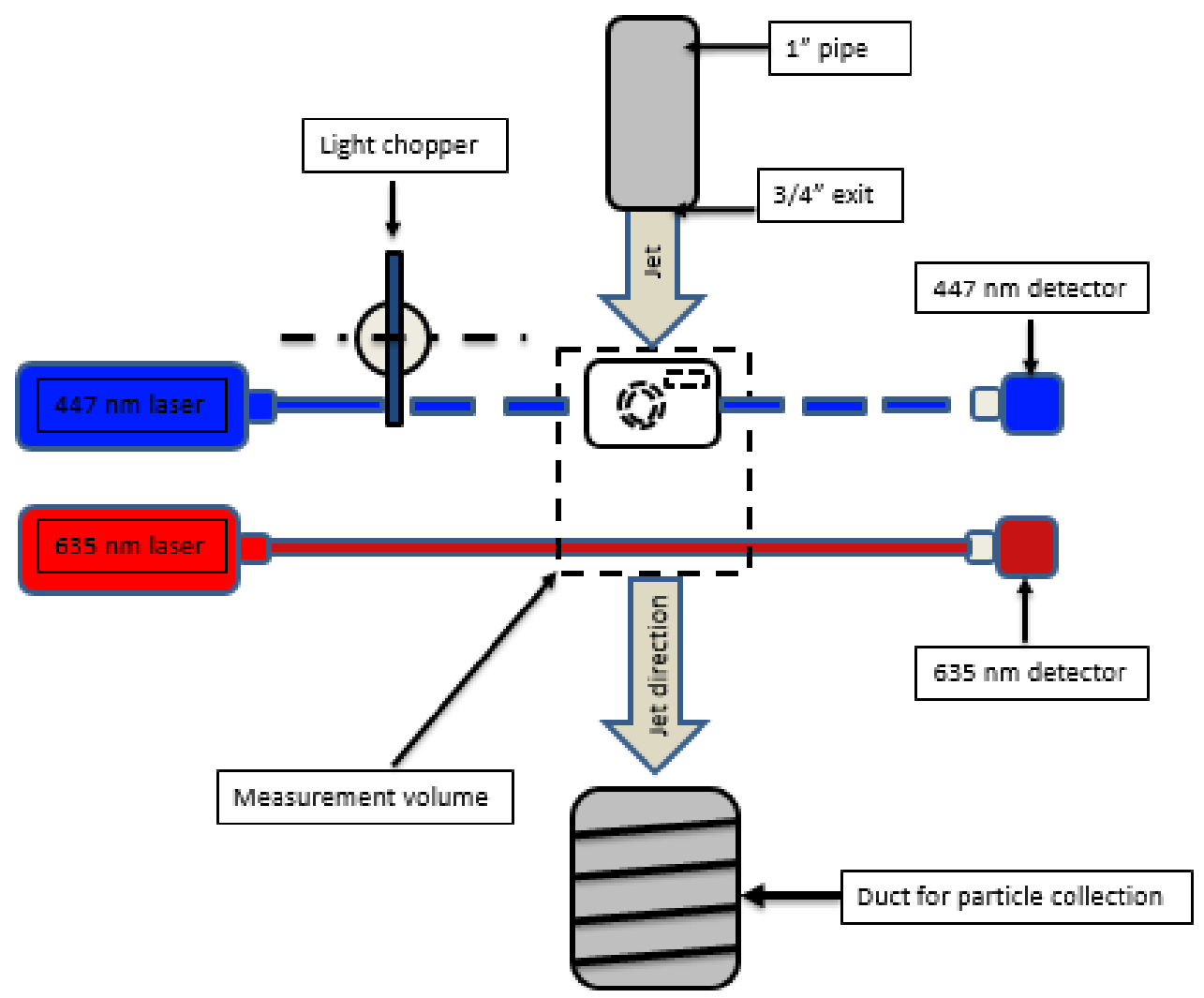

Figure 33: Schematic of the flow setup modified to obtain concentration validation measurements using a camera for image analysis. 


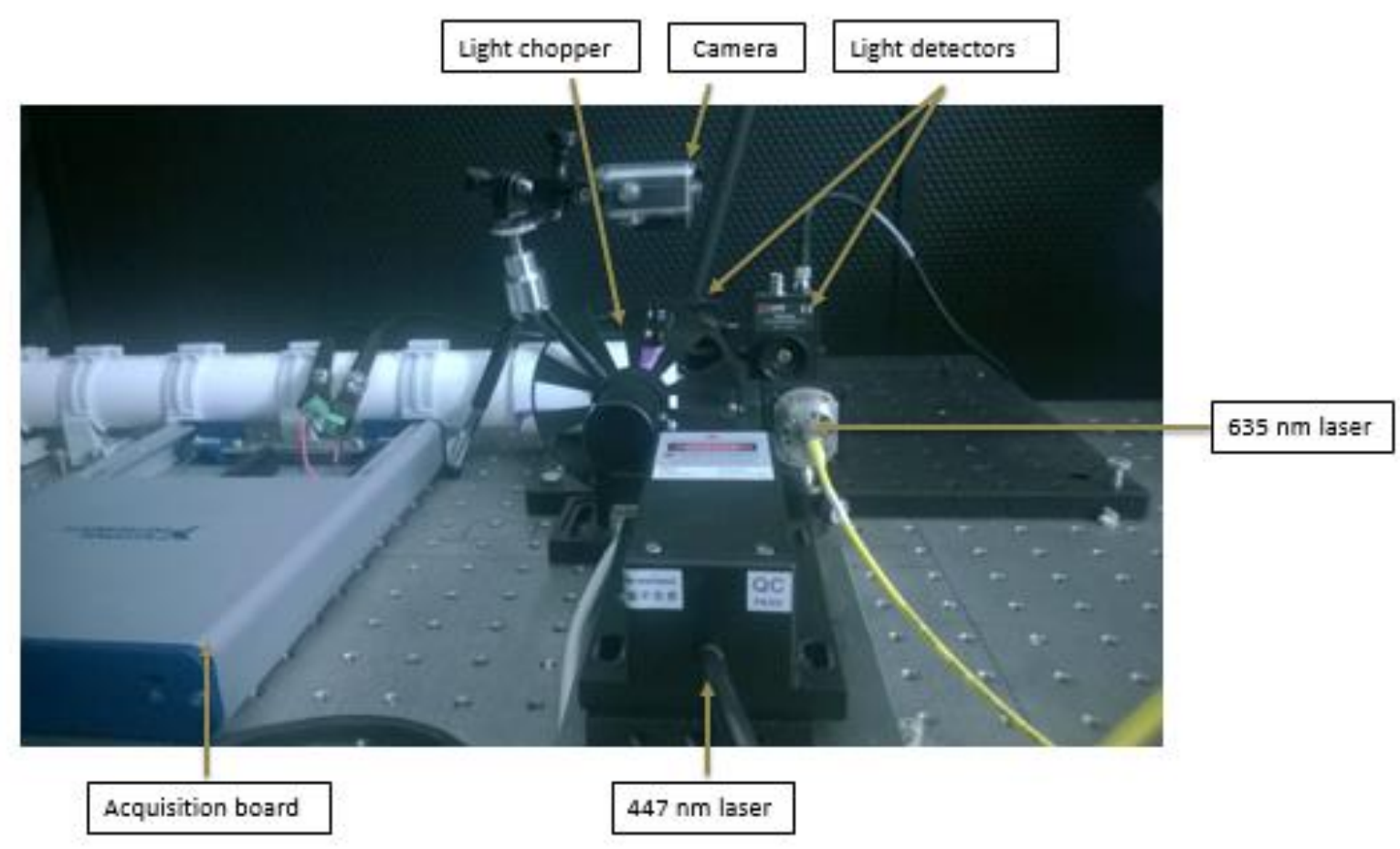

Figure 34: Actual image of the flow setup modified to obtain concentration validation measurements using a camera for image analysis.

Future work can also include the application of the technique to determine sizes and concentrations of seeded particles in a larger duct such as a blow down wind tunnel. p-H stabilized alumina particles can be dispersed in Water or Ethanol and the combined mixture can be seeded into the test facility using any commercial atomizer [28-29].

\section{Extension of Technique to Water Aerosols and Silica Particles}

For a measurement field such as an aircraft engine inlet, it becomes critical that the sensor provides maximum amount of information on particle size distributions with optimum utilization of on board resources. Thus it becomes critical to analyze the combination of light wavelength sources that will provide maximum information on particle size distributions within an allowable degree of ambiguity using a minimum number of light sources. The refractive index of silica particles and water aerosols was obtained from literature at $20^{\circ} \mathrm{C}[20$ - 
21] and the geometry was assumed spherical. Since silica particles and water droplets are in poly-dispersed by distribution, all diameters discussed in this section will be related to the Sauter mean diameter which is a diameter that gives the same volume to surface area ratio as a particle of interest. Utilizing the relations discussed in the measurement concepts earlier, ratios of the average extinction efficiencies at three selected wavelengths is plotted and shown in Fig. (35) and Fig. (36), below. By observing the distributions of extinction efficiencies ratios versus diameters, it can be concluded that a combination of $450 \mathrm{~nm}, 635 \mathrm{~nm}$ and $7000 \mathrm{~nm}$ lasers can be utilized to obtain information on diameters in the range of up to $7 \mu \mathrm{m}$ without any ambiguity. Beyond $7 \mu \mathrm{m}$, there exists an ambiguity in the diameter measurements due to the dependence of the ratio $R$ on standard deviations. However the maximum ambiguity calculated using Equation 6 for water aerosols and silica is $28 \%$. This ambiguity however is a result of the minimum number of wavelengths chosen. Increasing the number of wavelengths will reduce the ambiguity but will make the setup bulky.

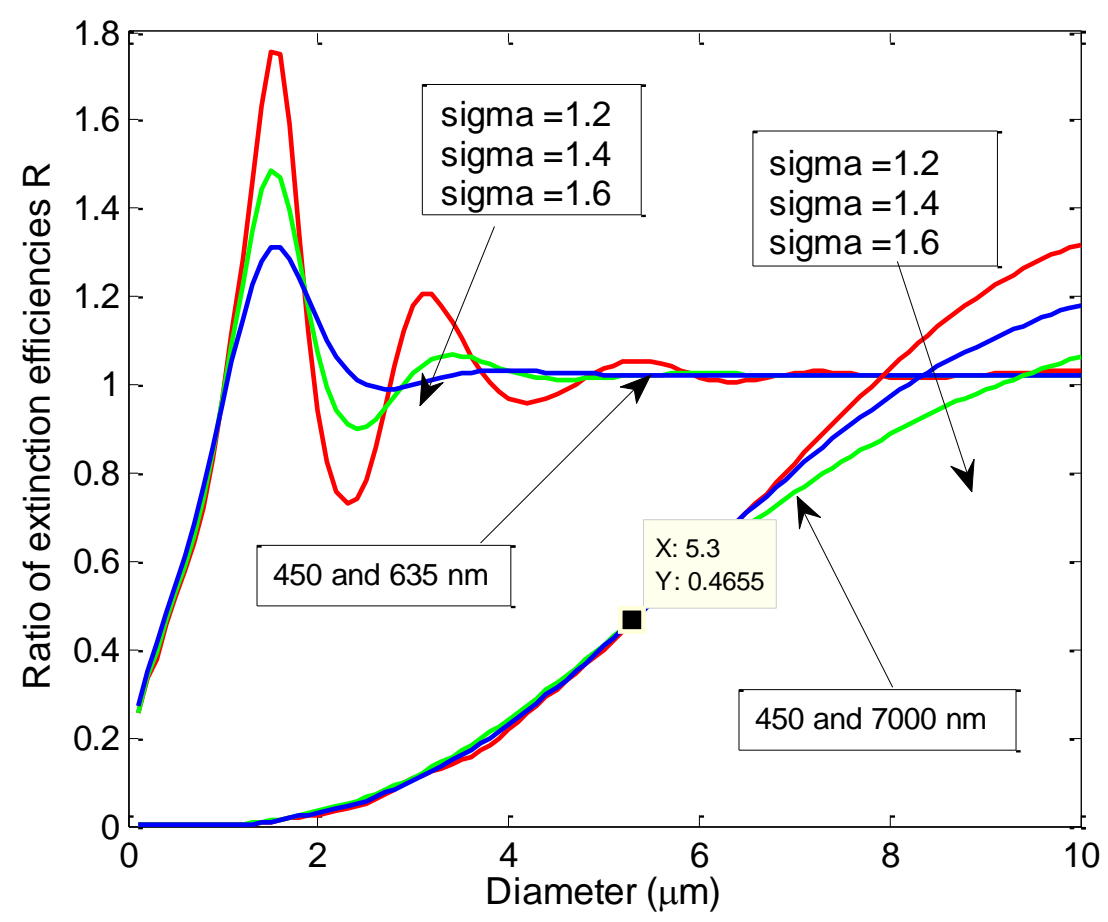

Figure 35: Ratio of extinction efficiencies for combinations of $635 \mathrm{~nm}, 450 \mathrm{~nm}$ and $7000 \mathrm{~nm}$ lasers using water aerosols. 


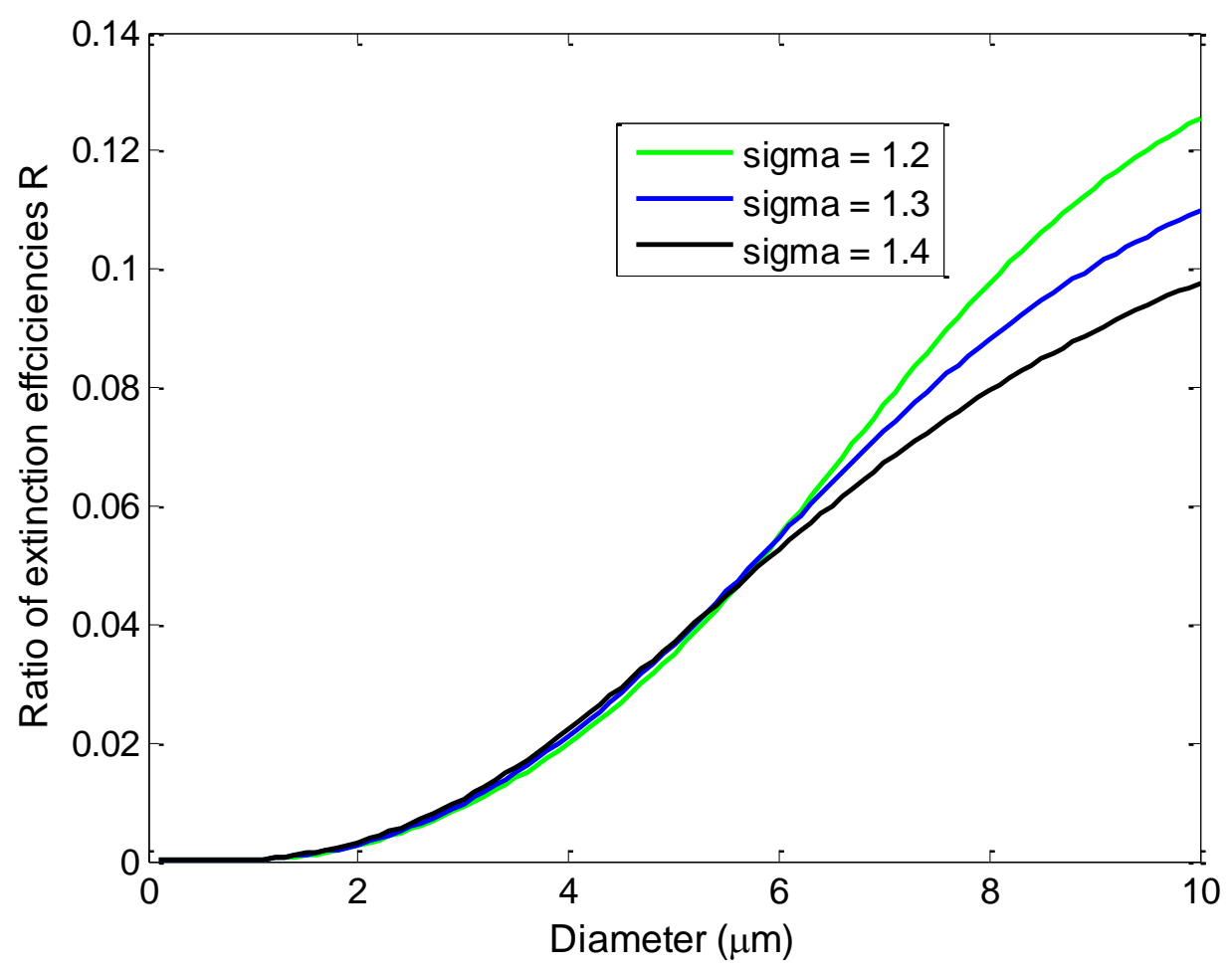

Figure 36: Ratio of extinction efficiencies for combinations of 450nm and 7000nm lasers using silica particles.

For the experimental setup, Silica particles can be seeded into the flow using and characterized using the extinction technique with sensors instrumented in a wind tunnel or on a stationary engine test bed. It would then be possible to analyze the performance of the measurement technique in realistic environments with instrument vibrations and study the influence of beam walk on the data sampled. Such tests would facilitate improvements to the ruggedness of the sensors employed and further increase the capability of the technique to be installed as a real time diagnostic tool for engine health monitoring.

All measurements described in this work have the capability to be made in real time. Database tables can be generated for particles of interest in the given size range as described in Figs. (3436), and virtual interfaces can be written to continuously measure the extinction efficiency ratio R. This measured data can be compared to the data in the lookup tables and diameters along 
with concentrations can be predicted in flight non-intrusively. Simulations and experiments have illustrated that it is feasible to use a neural network to obtain the parameters of a particle size distribution by training the neural network to decipher log normal particle data [27]. The method has an advantage of simplicity of use, instantaneous delivery of results and suitability for real time particle size analysis.

\section{Possible Sources of Error in Future On-Board Measurements}

While developing the technique, all possible sources of errors must also be taken into consideration. The most significant concerns currently include:

1. Engine vibrations causing beam steering and a resultant erroneous signal.

2. Beam deflections due to density variations across the engine inlet.

3. Temperature fluctuations causing a variation in refractive index.

4. Line of sight measurements not being representative of the flow field.

Engine vibrations will result in detector overfill. The vibrating laser beam will cause output signals to fall outside the detector active area. This may reduce the response of the photodetector resulting in a lower signal output and a diameter over-prediction. The response time of the detector may also increase as the photo-generated charge carriers will migrate to the detector active area through diffusion rather than drift. The effect of detector overfill can be mitigated by using a larger detector aperture along with collimation optics mounted near the aperture of the detector that will focus all the divergent light into a concentrated area in the active region of the detector. The beam deflection can be accounted for by utilizing adaptive optics that modulate the light beam before it enters the jet thereby resulting in a correction in the final transmitted light beam. The effects of refractive index variation with temperature can be mitigated by characterizing the effect of refractive index with temperature and using the refractive index information in an uncertainty analysis similar to the analysis described above 
thereby introducing correction factors for a given flow temperature. The measurements can be made flow representative by traversing the light source and detector system across the engine inlet. 


\section{CONCLUSION}

The work in this thesis describes the preliminary steps in developing a line of sight based optical extinction technique to measure particle sizes and concentrations in an aircraft engine inlet with an end goal of an on-board sensor. As an initial proof of concept experiment, Polystyrene particles in the size range of 1-10 $\mu \mathrm{m}$ were measured utilizing the wavelength multiplexed laser extinction technique using $447 \mathrm{~nm}$ and $635 \mathrm{~nm}$ lasers. Using the results obtained from measurements of polystyrene particle diameters and concentrations in water dispersions, the validity of the technique to measure particle sizes, standard deviations and number densities in the predetermined size range was proven. Along with the static measurements, the merit of the technique to size particles and obtain concentrations in a low speed jet was also demonstrated using Polystyrene particle samples in the range of 1-10 $\mu \mathrm{m}$. Results from the flow experiment showed good conformance with the actual particle diameters sampled.

An uncertainty analysis using the perturbation technique of individual measurement parameters was performed to obtain the errors in measurements due to sources of variability in the measurement system. The uncertainty analysis showed that the technique was able to resolve diameters with low errors for variation in measurement variables. The uncertainty in concentration measurements indicates an increasing uncertainty value for longer wavelength sources of light and would recommend using shorter light wavelengths to determine the concentration measurements from the extinct light.

Along with diameter measurements in flows, a setup to validate the concentrations measured from extinction measurements using the flow setup was discussed. The possibility of extending the technique to measure silica and water aerosol sizes under more realistic engine environments, with the optimum number of wavelength sources was also discussed. Results 
from such an experiment would provide Sauter mean diameters of non-spherical particles similar to desert sand ingested by aircraft engines.

The results and discussions from this thesis coupled with the non-intrusive principle of the optical extinction technique indicate good potential for real time diameter and concentration measurements and provides an impetus to carry out further research in the application of this technique to provide, in situ characterization of particles entering an aircraft engine inlet using an on board sensor. 


\section{NOMENCLATURE}

A

$C_{\text {ext }}$

$\mathbf{C}_{\mathbf{n}}$

D

D32

$\overline{\mathbf{D}}$

$\mathbf{H}_{\mathbf{z}}$

$\boldsymbol{I}_{t}$

$I_{o}$

L

$\mathbf{N}_{\mathbf{p}}$

$\mathbf{P}_{\mathbf{t}}$

$\mathbf{P}_{0}$

Q

$\overline{\mathbf{Q}}$

$\mathbf{R}_{\mathrm{ij}}$

SD

W

$\lambda_{\mathrm{i}}$

$\boldsymbol{\sigma}$

$\boldsymbol{\tau}_{\boldsymbol{i}}$
Current produced in Amperes

Total extinction cross section

Average number densities

Diameter

Sauter mean diameter

Mean diameter

Frequency response

Transmitted light intensity

Incident light intensity

Optical path length

Particle count

Transmitted power

Incident power

Extinction efficiency

Average extinction efficiency

Ratio of extinction efficiencies

Sensitivity parameter

Power in watts

Wavelength of incident light

Standard deviation

Transmissivity at wavelength $\lambda_{\mathrm{i}}$ 


\section{REFERENCES}

[1] L. Ma, L. Kranendonk, W. Cai, Y. Zhao, and J. Baba, "Application of simulated annealing for simultaneous retrieval of particle size distribution and refractive index," J. Aerosol Sci., vol. 40, no. 7, pp. 588-596, Jul. 2009.

[2] H. Horvath and C. Dellago, "On the accuracy of the size distribution information obtained from light extinction and scattering measurements-II. Case studies," Journal of Aerosol Science, vol. 24. pp. 143-154, 1993.

[3] W. Cai and L. Ma, "Information content of scattering measurements and characterization of spheroids," J. Aerosol Sci., vol. 39, no. 12, pp. 1032-1039, Dec. 2008 .

[4] D. J. Holve, "In Situ Optical Particle Sizing Technique,” J. Energy, vol. 4, no. 4, pp. 176-183, Jul. 1980.

[5] J. R. Hodkinson, "Particle sizing by means of the forward scattering lobe.," Appl. Opt., vol. 5, no. 5, pp. 839-44, May 1966.

[6] N. Damaschke, H. Nobach, N. Semidetnov, and C. Tropea, "Optical Particle Sizing in Backscatter," Appl. Opt., vol. 41, no. 27, p. 5713, Sep. 2002.

[7] P. Hull, I. Shepherd, and A. Hunt, "Modeling light scattering from Diesel soot particles," vol. 43, no. 17, pp. 3433-3441, 2004.

[8] J. Q. Lu, R. S. Brock, N. Carolina, T. J. Mcconnell, J. F. Ojeda, K. M. Jacobs, and X. $\mathrm{Hu}$, "Angle-resolved Mueller matrix study of light scattering by B-cells at three wavelengths of 442, 633, and $850 \mathrm{~nm}$," vol. 12, no. June 2007, pp. 1-9, 2014.

[9] L. Ma and R. K. Hanson, "Measurement of aerosol size distribution functions by wavelength-multiplexed laser extinction," Appl. Phys. B, vol. 81, pp. 567-576, 2005.

[10] R. J. Litchford, F. Sun, J. D. Few, and J. W. L. Lewis, "Optical Measurement of Gas Engine Soot Particle Effluents,” vol. 120, no. January 1998, 2014.

[11] M. E. van Dongen, H. J. Smolders, C. J. Braun, C. a Snoeijs, and J. F. Willems, "Spectral light extinction to characterize fast fog formation.," Appl. Opt., vol. 33, no. 10, pp. 1980-8, Apr. 1994.

[12] G. Ramachandran and D. Leith, "Extraction of Aerosol-Size Distributions from Multispectral Light Extinction Data," Aerosol Sci. Technol., vol. 17, no. 4, pp. 303325, Jan. 1992.

[13] M. Schatz, T. Eberle, M. Grübel, J. Starzmann, D. M. Vogt, and N. Suerken, "TwoPhase Flow Modeling and Measurements in Low-Pressure Turbines-Part II: Turbine Wetness Measurement and Comparison to Computational Fluid DynamicsPredictions," J. Eng. Gas Turbines Power, vol. 137, no. 4, p. 042603, Oct. 2014. 
[14] X.-S. Cai and N.-N. Wang, "Determination of particle size distribution using the light extinction method," Adv. Powder Technol., vol. 3, no. 3, pp. 153-161, Jan. 1992.

[15] P. T. Walters, "Practical applications of inverting spectral turbidity data to provide aerosol size distributions.," Appl. Opt., vol. 19, no. 14, pp. 2353-65, Jul. 1980.

[16] S. Twomey and H. B. Howell, "Some Aspects of the Optical Estimation of Microstructure in Fog and Cloud," Appl. Opt, vol. 6, no. 12, pp. 2125-2131, 1967.

[17] H. J. Smolders and M. E. H. van Dongen, "Shock wave structure in a mixture of gas, vapour and droplets," Shock Waves, vol. 2, no. 4, pp. 255-267, Dec. 1992.

[18] M. Su, F. Xu, X. Cai, K. Ren, and J. Shen, "Optimization of regularization parameter of inversion in particle sizing using light extinction method," China Particuology, vol. 5, no. 4, pp. 295-299, Aug. 2007.

[19] X. Sun, D. J. Ewing, and L. Ma, "A laser extinction based sensor for simultaneous droplet size and vapor measurement," Particuology, vol. 10, no. 1, pp. 9-16, Feb. 2012.

[20] K. S. Shifrin and I. G. Zolotov, "Determination of the Aerosol Particle Size distribution from simultaneous data on spectral attenuation and the small-angle phase function," Appl. Opt. vol. 36, no. 24, pp. 6047-6056, February 1997.

[21] K. S. Shifrin and I. G. Zolotov, "Spectral attenuation and aerosol particle size distribution," Appl. Opt, vol. 35, no. 12, pp. 2114-2124, 1996.

[22] D. Advanced, U. S. A. Aviation, and M. C. Under, "Sandblaster 2 Support of SeeThrough Technologies for Particulate Brownout Task 5 Final Technical Report Sandblaster 2 Support of See-Through Technologies for Particulate Brownout Task 5 Final Technical Report," no. 110565, 2007.

[23] N. Sultanova, S. Kasarova, and I. Nikolov, "Dispersion Properties of Optical Polymers," vol. 116, no. 4, pp. 585-587, 2009.

[24] R. J. Moffat, "Describing the Uncertainties in Experimental Results," Experimental Thermal and Fluid Sciences, vol.1, no. 1, pp. 3-17, 1988.

[25] I.H.Malitson. Introduction, "Interspecimen Comparison of the Refractive Index of Fused Silica", Journal of the Optical Society of America, vol. 55, no. 10, pp.1205 1965.

[26] G. M. Hale and M. R. Querry, "Optical Constants of Water in the 200-nm to 200microm Wavelength Region.," Appl. Opt., vol. 12, no. 3, pp. 555-63, Mar. 1973.

[27] M. Li, T. Frette, and D. Wilkinson, "Particle Size Distribution Determination from Specral ExtinctionUsing Neural Networks,"Ind. Eng. Chem.Res, pp. 4615-4622, 2001.

[28] M. Y. Weirlet, G. J. Skoch, and C. Oh, "Demonstration of a Stabilized Alumina / Ethanol Colloidal Dispersion Technique for Seeding High Temperature Air 
Flows,'ICIASF , International Congress on Instrumentation in Aerospace Simulation Facilities, pp. 1-9, 1995.

[29] J. H. Wernet and M. P. Wernet, "Stabilized Alumina/Ethanol Colloidal Dispersion for Seeding High Temperature Air Flows", Symposium on Laser Anemometry: Advances and Applications Sponsored by American Society for Mechanical Engineers Lake Tahoe, Nevada , June 19-23 , 2004.

[30] Kryzszystof Markowicz, "Codes,"

http://www.igf.fuw.edu.pl/meteo/stacja/index_english.php. [Accessed: 03-Mar-2014]. 


\section{APPENDIX A：DISAMBIGUATION OF DIAMETERS OBTAINED}

Consider the plot of $\mathrm{R}$ vs diameter for polystyrene particles at a standard deviation of 1.033 as shown in Figure 6. The true mean diameter of the particle sample is $3.9 \mu \mathrm{m}$. An $R$ value of 1.11 was obtained experimentally using the two selected wavelength combination of $450 \mathrm{~nm}$ and $635 \mathrm{~nm}$. This ratio however could lead to 7 possible diameters due to the oscillatory behavior of the $R$ vs diameter curve. Since the diameter of the test particles were known before hand, an analysis was performed to show that the increase in spectral width results a singular value corresponding to the diameter tested. The increase of spectral width was not achieved physically but was theoretically preformed and corresponding values of $\mathrm{R}$ were calculated using the Mie extinction code. As expected, the increase in spectral width reduces the number of possible diameters and at a wavelength combination of $10 \mu \mathrm{m}$ and $450 \mathrm{~nm}$, there exists only one unique value of diameter for the given ratio which corresponds to a $3.9 \mu \mathrm{m}$ diameter. This is demonstrated in Figs. (A1-A2), where the diameters are shortlisted to a unique value of 3.9 $\mu \mathrm{m}$.

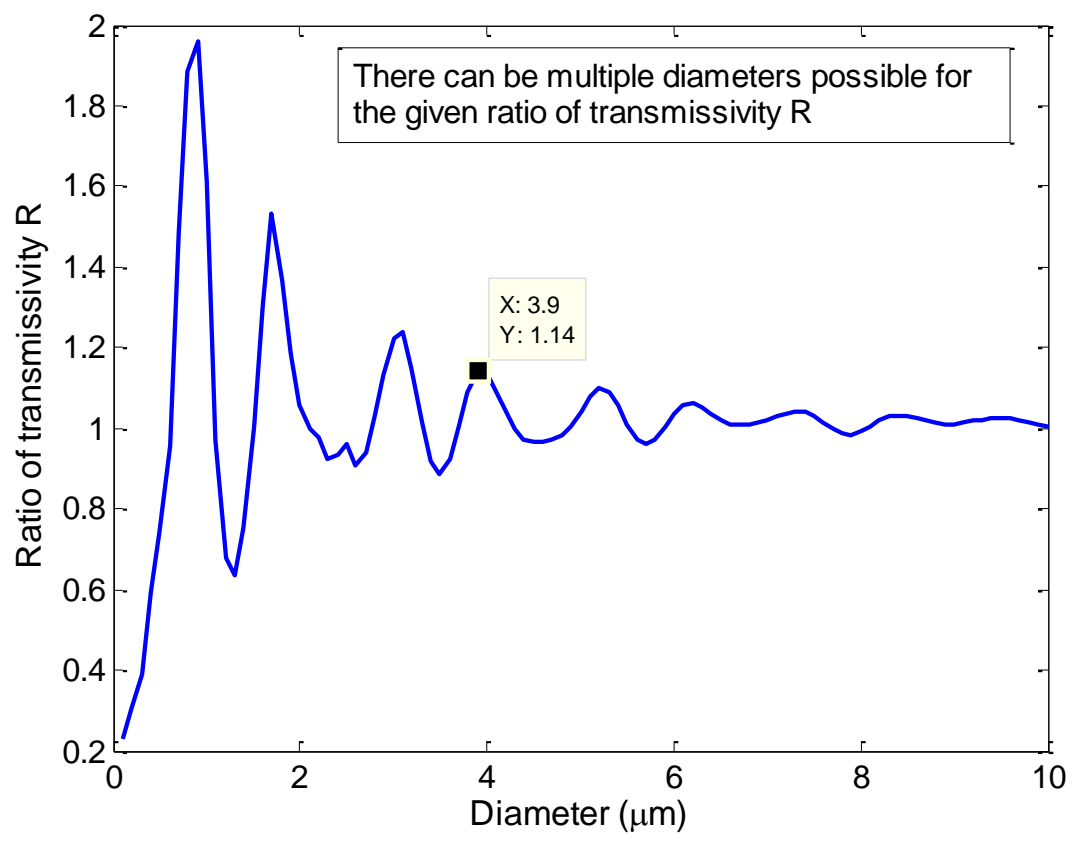

Figure A1: Ratio of transmissivity $R$ versus diameter using $635 \mathrm{~nm}$ and $450 \mathrm{~nm}$ laser for a standard deviation of 1.033 . 


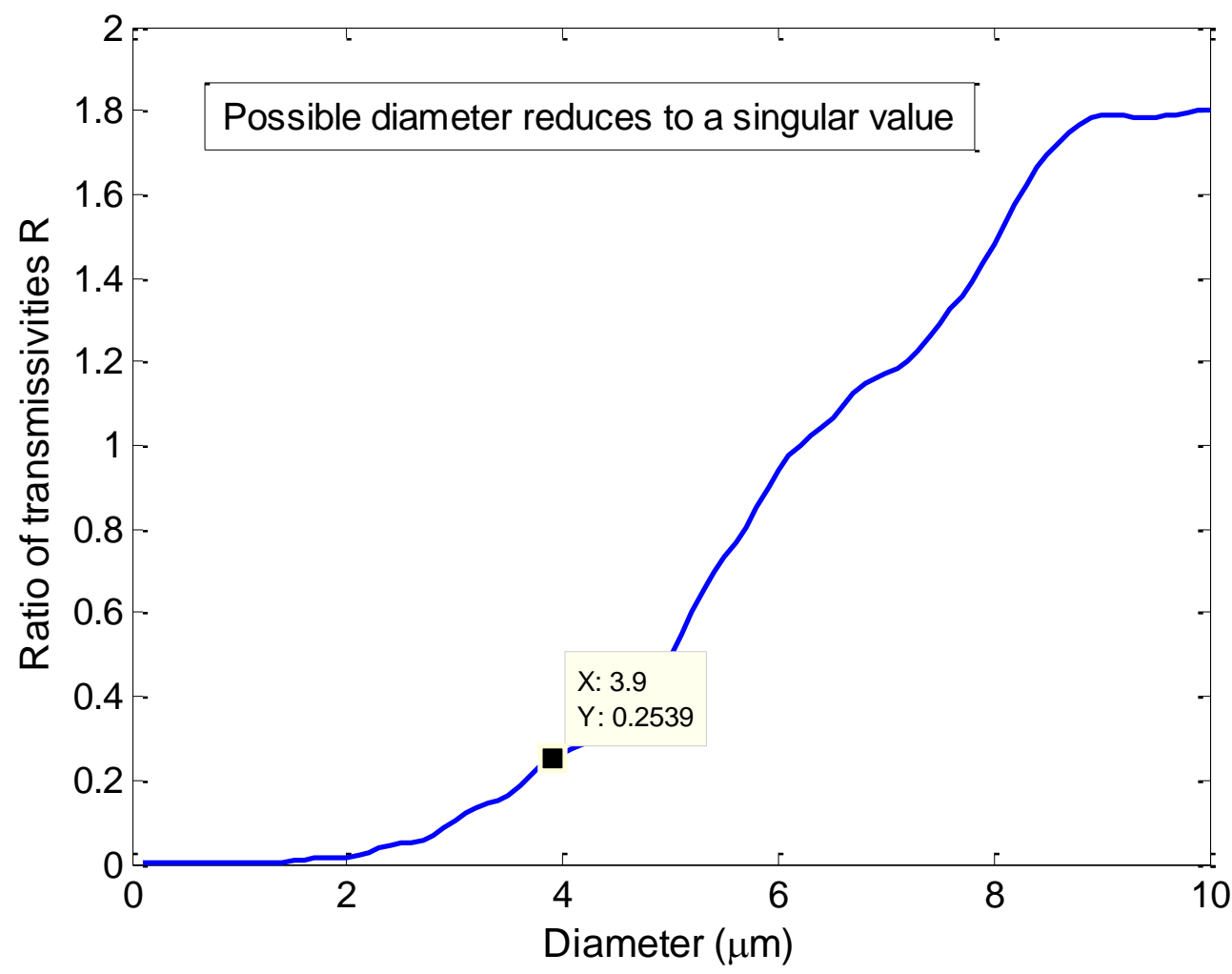

Figure A2: Ratio of transmissivity $R$ vs diameter using $450 \mathrm{~nm}$ and $10000 \mathrm{~nm}$ laser for a standard deviation of $\mathbf{1 . 0 3 3}$. Note the reduction in the number of possible diameters compared to Figure A1. 


\section{APPENDIX B: CODES FOR MIE EXTINCTION APPLIED TO PARTICLE SIZE DISTRIBUTIONS}

The original code for MIE extinction modified in this work was written by Krzszystof Markowicz [30].

\section{Code for MIE Extinction}

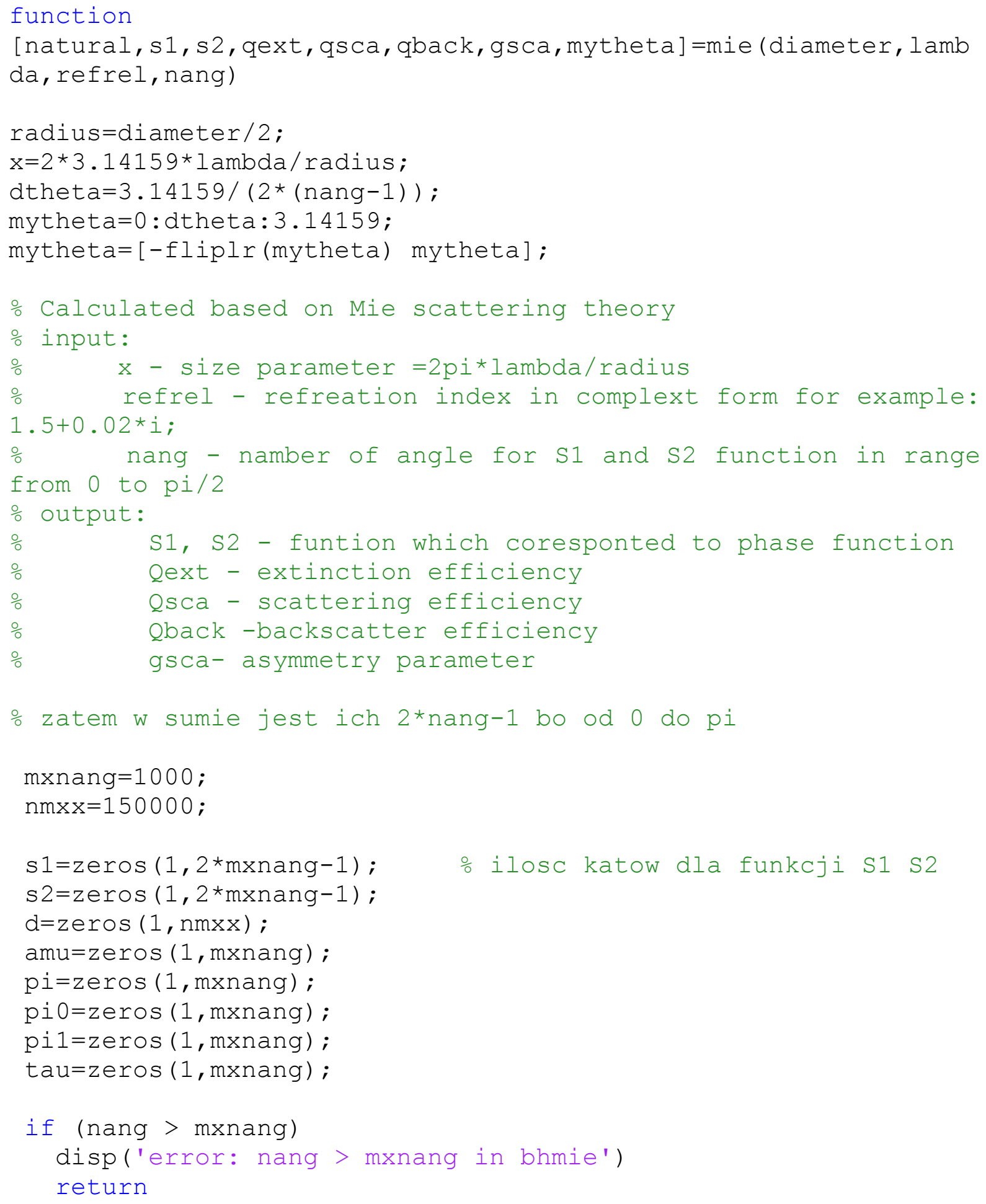


o beginning with initial value $(0 ., 0$.$) at J=$ NMX

$\frac{\circ}{\circ}$

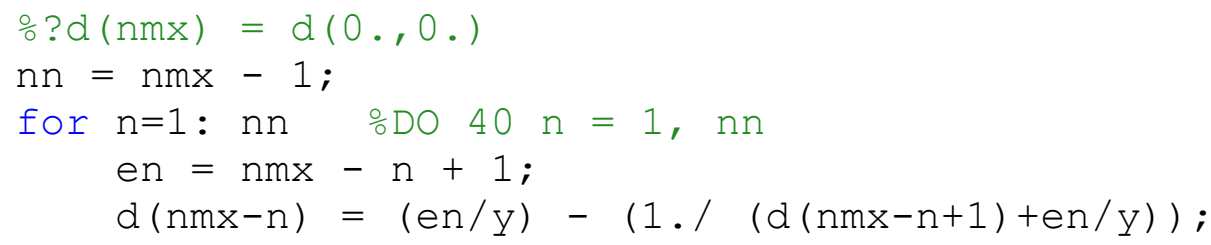

end endfor $\div 40$ CONTINUE

$\frac{0}{0}$

\%*** Riccati-Bessel functions with real argument $\mathrm{X}$

\% calculated by upward recurrence

$\frac{0}{\circ}$

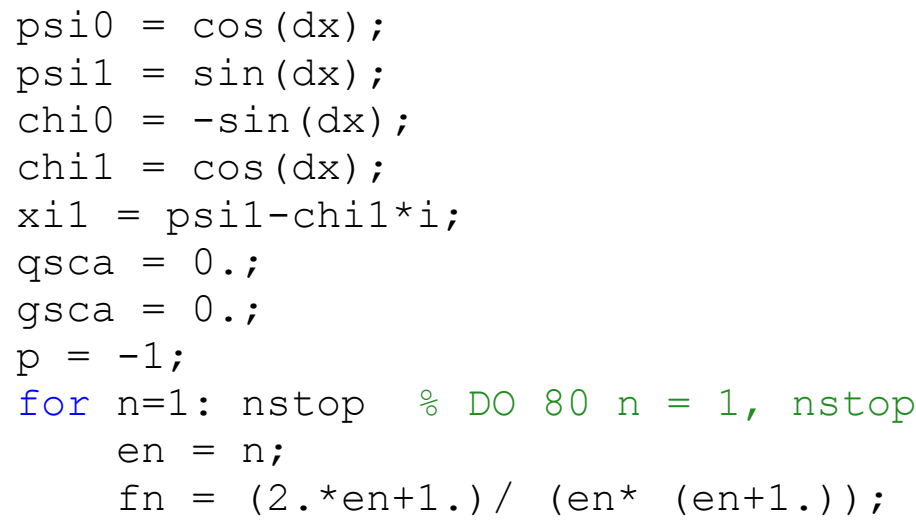

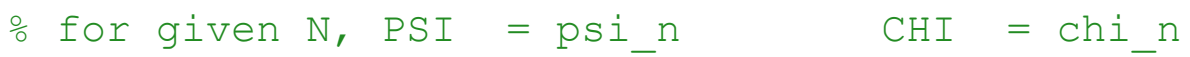

$\frac{\text { PSII }}{\circ}=$ psi_ $\{\mathrm{n}-1\} \quad \operatorname{CHII}=\mathrm{chi}\{\mathrm{n}-1\}$

PSIO $=$ psi_ $\{n-2\} \quad$ CHIO $=$ chi $\{n-2\}$

o Calculate psi $n$ and chin

psi $=\left(2 .{ }^{*} e n-1.\right){ }^{*}$ psil $/ d x-p s i 0 ;$

$\operatorname{chi}=\left(2 .{ }^{*} e n-1.\right){ }^{*} \operatorname{chil} / d x-\operatorname{chi0}$;

$x i=p s i-c h i * i$

$\%$

\%*** Store previous values of AN and BN for use

\% in computation of $g=\langle\cos$ (theta) $\rangle$

if $(\mathrm{n}>1)$ \%then begin

an $1=a n ;$

bn $1=$ bn;

end $\frac{\circ}{\circ}$ dif

$\circ$

\%*** Compute $\mathrm{AN}$ and $\mathrm{BN}$ :

$$
\begin{aligned}
& a_{n}=(d(n) / d r e f r l+e n / d x) * p s i-p s i l ; \\
& \text { an }=a n /((d(n) / d r e f r l+e n / d x) * x i-x i l) ; \\
& b n=(d r e f r l * d(n)+e n / d x) * p s i-p s i l ; \\
& b n=b n /((d r e f r l * d(n)+e n / d x) * x i-x i l) ;
\end{aligned}
$$

$\frac{0}{0}$

ㅇ* Augment sums for Qsca and $g=\langle\cos$ (theta) $\rangle$

$$
\begin{aligned}
& \mathrm{qsca}=\mathrm{qsca}+\left(2 .{ }^{\star} e n+1 .\right) \star\left(\operatorname{abs}(\mathrm{an})^{\wedge} 2+\mathrm{abs}(\mathrm{bn})^{\wedge} 2\right) ; \\
& \text { gsca }=\text { gsca }+\left(\left(2 *^{\star} e n+1 .\right) /\left(e^{\star}(e n+1 .)\right)\right) \star \ldots \\
& (\operatorname{real}(a n) * \operatorname{real}(b n)+i m a g(a n) * i m a g(b n)) \text {; } \\
& \text { if }(n>1) \text { othen begin } \\
& \text { gsca }=\operatorname{gsca}+((e n-1 .) *(e n+1 .) / e n) * \ldots
\end{aligned}
$$




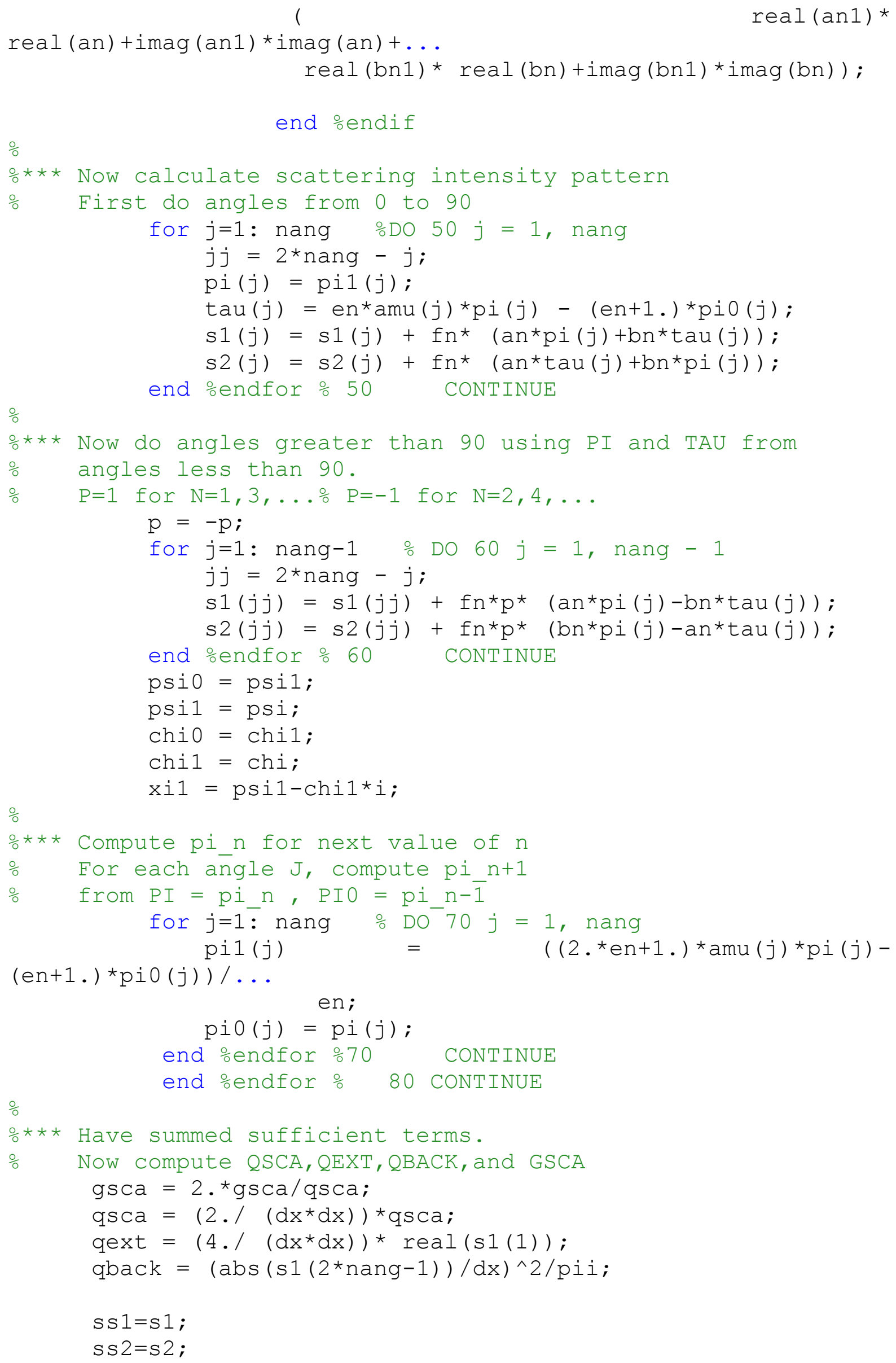




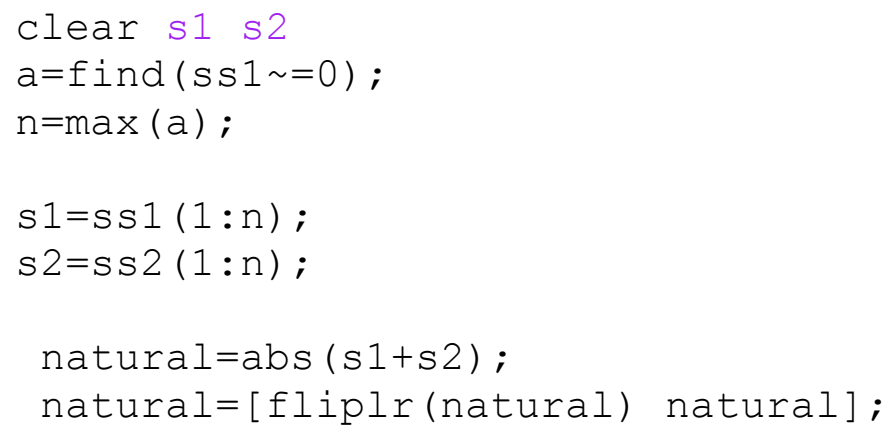

\section{Code for Applying MIE extinction to Particle Size Distributions}

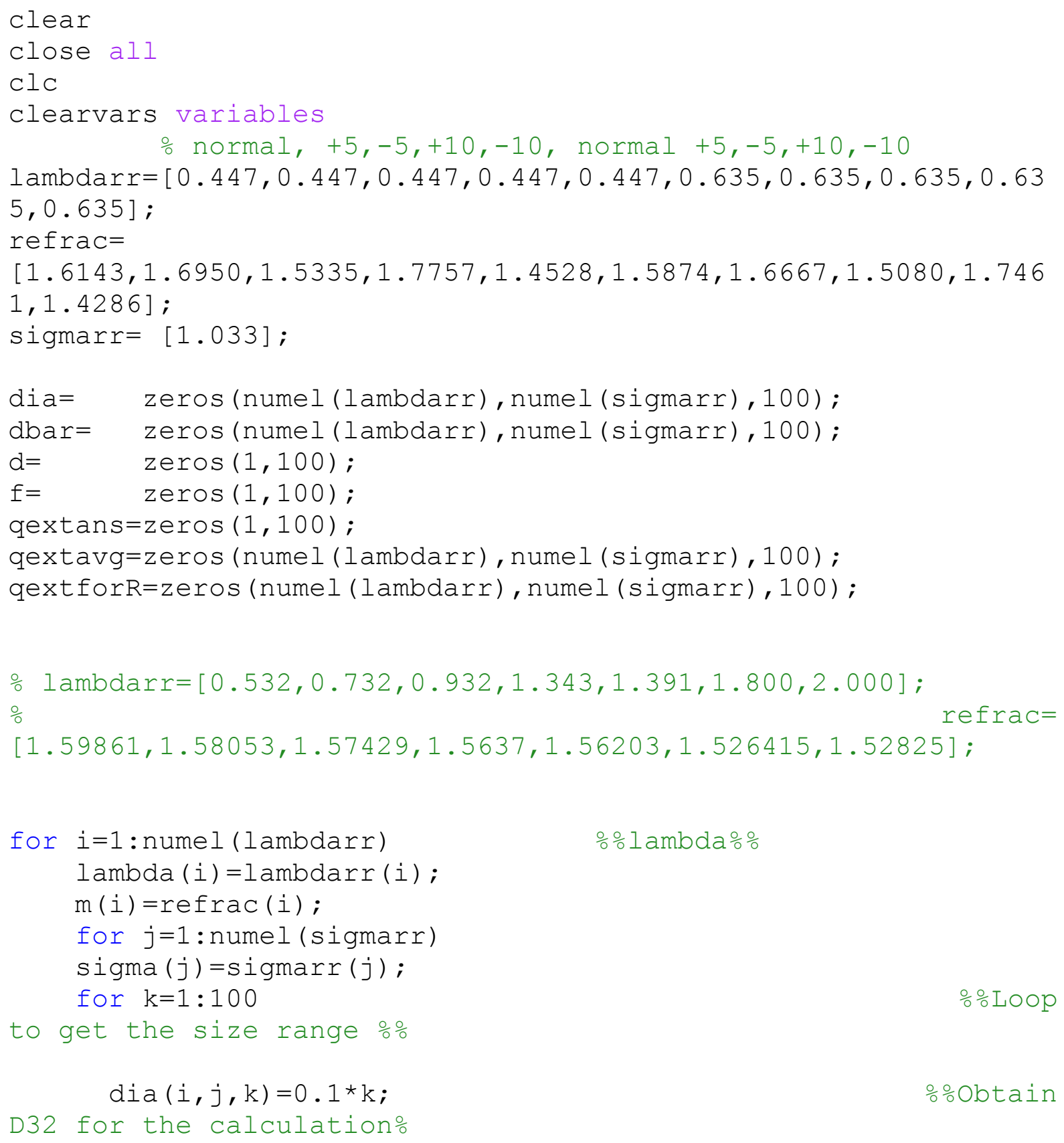


$\operatorname{dbar}(i, j, k)=\exp (\log (\operatorname{dia}(i, j, k))-$

$\left.\left(5 / 2 *\left(\left(\log (\operatorname{sigma}(j))^{\wedge} 2\right)\right)\right)\right)$; $\frac{\circ}{0}$ Obtain the meano\%

for $1=1: 100$

응 Loop

to get the $\mathrm{D}$ for the $\mathrm{PDF} \%$ \%

$d(1)=0.1 * 1$;

$\because$ Use

this $\mathrm{D}$ to get the $\mathrm{PDF} \%$

$f(1)=(1 / \operatorname{sqrt}(2 * \mathrm{pi}) / \mathrm{d}(1) / \log (\operatorname{sigma}(j))) * \exp (-$

$\left.\left(1 / 2 /(\log (\operatorname{sigma}(j)))^{\wedge} 2 *((\log (d(1))-\log (\operatorname{dbar}(i, j, k))) \wedge 2)\right)\right) ;$

\% $\%$ Generate Log Normal Function\%

$[\sim, \sim, \sim$, qext $, \sim, \sim, \sim, \sim]=\operatorname{mie}(\mathrm{d}(1), \operatorname{lambda}(i), \mathrm{m}(\mathrm{i}), 180)$;

$\circ \frac{\circ}{0}$ For each D calculate the Qext $\% \circ$

qextans $(l)=$ qext

end

Get average extinction efficiency for the given D32\%

deltad=0.1;

qextavg $(i, j, k)=\left(\operatorname{trapz}\left(f .{ }^{*}\right.\right.$ qextans.*d.^2)*deltad) / (trapz $(f . * d . \wedge 2$

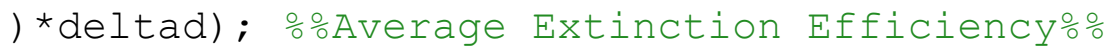

qextforR $(i, j, k)=\operatorname{qextavg}(i, j, k)$;

end

end

end 